\title{
Transformation of Alkynes into $\alpha$ - or $\beta$-Difluorinated Alkyl Azides by an Efficient One-Pot Two-Step Procedure
}

\author{
Huaizhi Li, ${ }^{\dagger} *$ Bhoomireddy Rajendra Prasad Reddy ${ }^{\dagger}$ and Xihe $\mathrm{Bi}^{*},+, *$ \\ ${ }^{\dagger}$ Jilin Province Key Laboratory of Organic Functional Molecular Design \& Synthesis, \\ Department of Chemistry, Northeast Normal University, Changchun 130024, China \\ \$ State Key Laboratory of Elemento-Organic Chemistry, Nankai University, Tianjin \\ 300071, China \\ Corresponding Authors: \\ *bixh507@nenu.edu.cn
}

\section{Table of contents}

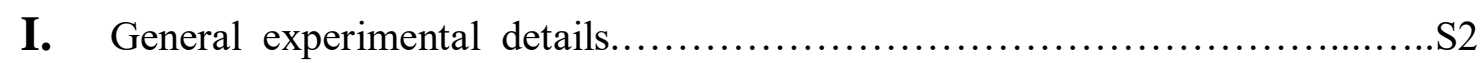

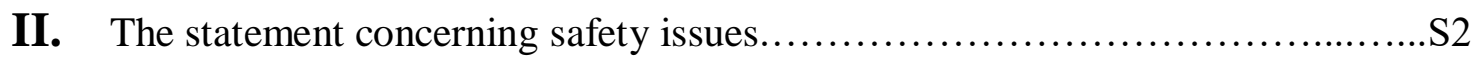

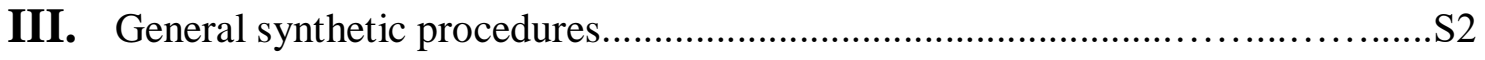

IV. Characterization data for the products..................................... 4

V. Elemental Analysis of Compound 3z................................... 15

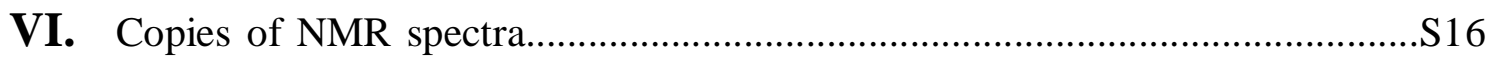




\section{General experimental details}

All reagents and solvents were purchased from commercial sources and used without purification unless otherwise stated. The products were purified by column chromatography over silica gel (200-400 size). The ${ }^{1} \mathrm{H},{ }^{13} \mathrm{C}$ and ${ }^{19} \mathrm{~F}-\mathrm{NMR}$ were recorded in $\mathrm{CDCl}_{3}$ using Bruker AV-600 (600 $\mathrm{MHz}$ for ${ }^{1} \mathrm{H}, 150 \mathrm{MHz}$ for ${ }^{13} \mathrm{C}$ and $564 \mathrm{MHz}$ for ${ }^{19} \mathrm{~F}$ ) and Agilent (Varian)-500 (500 MHz for ${ }^{1} \mathrm{H}$, $125 \mathrm{MHz}$ for ${ }^{13} \mathrm{C}$ and $470 \mathrm{MHz}$ for ${ }^{19} \mathrm{~F}$ ) spectrometers using TMS as internal standard. Coupling constants are given in hertz. The following abbreviations are used: s, singlet; d, doublet; t, triplet; q, quadruplet, quint., quintet, $\mathrm{m}$, multiplet, dd, doublet of doublet, tt, triplet of triplet, td, triplet of doublet. Mass spectra were recorded on TSQ 8000 Evo by using EI method. High resolution mass spectra (HRMS) were recorded on Bruck microTof by using ESI method. Elemental Analysis were recorded on euro Vector EA 3000.

\section{The statement concerning safety issues}

(1) Organic azides are known to be potentially explosive compounds. While we did not encounter any issues during their synthesis, proper precautions were taken. All azidation reactions and subsequent workups should be performed behind a blast shield. Once isolated, organic azides should be stored below room temperature and away from sources of heat, light, pressure, and shock. We have never experienced a safety problem with these materials.

(2) Pyridine $\cdot \mathrm{HF}$ is a corrosive and toxic substance that will corrode glassware. Safe handling can be conducted with plastic syringes and metal needles, with $\mathrm{NaHCO}_{3}$ (aq.) or $\mathrm{NaOH}$ (aq.) employed to quench excess HF. Though reactions should not be conducted in glassware when employing pyridine $\bullet \mathrm{HF}$, glassware may be used to quench reactions provided sufficient quantities of the base are present. Always handle pyridine•HF while wearing gloves and in a fume hood. As a precautionary measure, have calcium gluconate gel nearby and apply immediately and liberally on skin exposed to HF.

\section{General synthetic procedures}

General procedure A for hydroazidation/gem-difluorination (2a-2u, 3s-3z, 3ab-3ae): To a 15 $\mathrm{mL}$ sealed tube were added alkyne $1(1.0 \mathrm{mmol})$ and dimethylsulfoxide $(2.0 \mathrm{~mL})$. Then trimethylsilyl azide $(263 \mu \mathrm{L}, 2.0 \mathrm{mmol})$ and water $(36 \mathrm{mg}, 2.0 \mathrm{mmol})$ were added, respectively. Finally, the freshly prepared $\mathrm{AgN}_{3}(8 \mathrm{mg}, 5 \mathrm{~mol} \%)$ was added. Then the sealed tube is placed in an oil bath at $80{ }^{\circ} \mathrm{C}$. After the completion of the reaction (as evidenced by TLC), the reaction mixture was cooled to room temperature. Further removal of Ag catalyst and DMSO, the resulting mixture was concentrated and taken up by dichloromethane $(3 \times 30 \mathrm{~mL})$. The organic layer was washed with brine $(3 \times 40 \mathrm{~mL})$, dried over $\mathrm{MgSO}_{4}$ and concentrated. To the residue were added DCM (3 mL), Py·HF (496 mg, $5.0 \mathrm{mmol}$ ), and PIDA (484 mg, $1.5 \mathrm{mmol}$ ) in a $15 \mathrm{~mL}$ of Schlenk tube under air. The reaction mixture was stirred at $25{ }^{\circ} \mathrm{C}$ for $1 \mathrm{~min}$. After completion of the reaction, the combined filtrate was washed with a saturated aqueous solution of $\mathrm{NaHCO}_{3}(2 \times 50$ $\mathrm{mL}$ ). The organic layer was dried with anhydrous $\mathrm{MgSO}_{4}$ and concentrated under reduced pressure with low temperature. 
Note: Products can't be analyzed by HRMS, probably due to the decomposition.

General procedure B for the synthesis of 4: A $15 \mathrm{~mL}$ sealed tube equipped with magnetic stir bar was charged with $\mathbf{2 h}$ (99 mg, $0.5 \mathrm{mmol}), \mathrm{PPh}_{3}(263 \mathrm{mg}, 1.0 \mathrm{mmol}), \mathrm{H}_{2} \mathrm{O}$ (200 $\mu \mathrm{l})$, and THF $(2.0 \mathrm{ml})$. The reaction mixture was stirred at $50{ }^{\circ} \mathrm{C}$ for $3 \mathrm{~h}$. After, the reaction mixture was cooled to room temperature and $10 \mathrm{~mL}$ EtOAc was added to the mixture and layers partitioned. The aqueous layer was extracted with EtOAc $(2 \times 10$ $\mathrm{mL}$ ) and the combined organic extracts were washed with brine, dried over anhydrus $\mathrm{Na}_{2} \mathrm{SO}_{4}$, and concentrated under reduced pressure. The resulting crude product was purified by column chromatography using petroleum ether as eluent to afford 4 in a $97 \%$ yield as a colorless oil.

General procedure $\mathbf{C}$ for the synthesis of 5: A $15 \mathrm{~mL}$ sealed tube equipped with magnetic stir bar was charged with $\mathbf{2 h}$ (99 $\mathrm{mg}, 0.5 \mathrm{mmol}$ ), DMAD (86 $\mathrm{mg}, 0.6 \mathrm{mmol}$ ), and water $(8.0 \mathrm{~mL})$. The reaction mixture was stirred at $70{ }^{\circ} \mathrm{C}$ for $8 \mathrm{~h}$. After, the reaction mixture was cooled to room temperature and $10 \mathrm{~mL}$ EtOAc was added to the mixture and layers partitioned. The aqueous layer was extracted with EtOAc $(2 \times 10 \mathrm{~mL})$ and the combined organic extracts were washed with brine, dried over anhydrus $\mathrm{Na}_{2} \mathrm{SO}_{4}$, and concentrated under reduced pressure. The resulting crude product was purified by column chromatography using petroleum ether as eluent to afford 5 in a $94 \%$ yield as a colorless solid.

$$
\text { DMAD = Dimethyl acetylenedicarboxylate }
$$

\section{General procedure $D$ for the synthesis of $\left[D_{2}\right]-6$ :}

To a $15 \mathrm{~mL}$ sealed tube were added alkyne $1(1.0 \mathrm{mmol})$ and dimethylsulfoxide (2.0 $\mathrm{mL})$. Then trimethylsilyl azide $(263 \mu \mathrm{L}, 2.0 \mathrm{mmol})$ and $\mathrm{D}_{2} \mathrm{O}(40 \mathrm{mg}, 2.0 \mathrm{mmol})$ were added, respectively. Finally, the freshly prepared white $\mathrm{AgN}_{3}(8 \mathrm{mg}, 5 \mathrm{~mol} \%$ ) was added and the reaction mixture was stirred at $80{ }^{\circ} \mathrm{C}$. After the completion of the reaction (as evidenced by TLC), the reaction mixture was cooled to room temperature, quenched with water $(10 \mathrm{~mL})$ and extracted with EtOAc $(2 \times 25 \mathrm{~mL})$. The organic layer was washed with brine, dried over anhydrus $\mathrm{Na}_{2} \mathrm{SO}_{4}$ and the solvent was removed under reduced pressure. The resulting deuterated product was purified by a silica gel column chromatography using petroleum ether as the eluent to afford deuterated vinyl azide $\left[\mathbf{D}_{2}\right]-6$ in a $81 \%$ yield as a yellow oil.

The extent of deuterium incorporation in $\left[\mathbf{D}_{2}\right]-6(\sim 60 \% \mathrm{D})$ was determined from the relative integration of the vinylic protons at $\delta 5.37-5.35(\mathrm{~m}, 0.49 \mathrm{H})$, and $4.90-4.88$ $(\mathrm{m}, 0.27 \mathrm{H})$. 


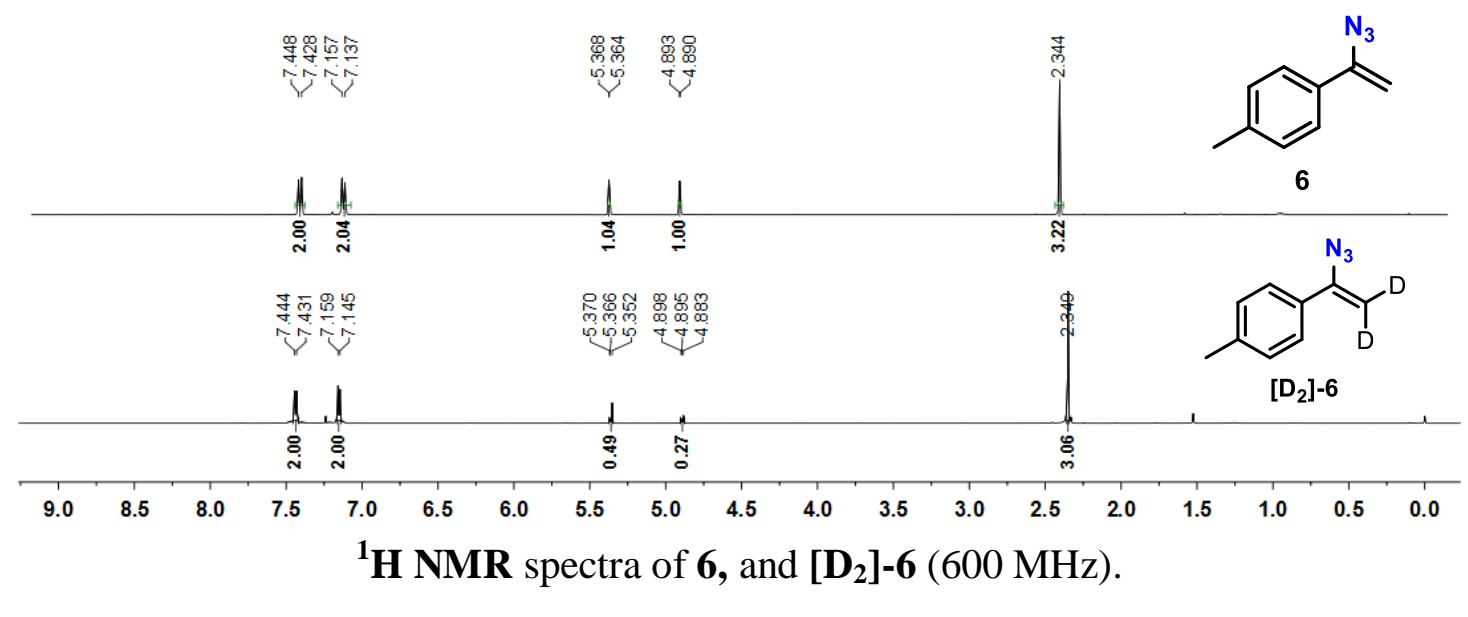

General procedure $\mathbf{E}$ for the synthesis of $\left[\mathrm{D}_{2}\right]-3 \mathrm{y}$ :

To a $15 \mathrm{~mL}$ flask were added deuterated vinyl azide, Py·HF (496 mg, $5.0 \mathrm{mmol}$ ), PIDA (484 mg, $1.5 \mathrm{mmol}$ ), and DCM (3 mL). The reaction mixture was stirred at 25 ${ }^{\circ} \mathrm{C}$ for $1 \mathrm{~min}$. After completion of the reaction, solvent was removed under reduced pressure, the residue was separated by column chromatography using petrolium ether as eluent to afford $\left[\mathbf{D}_{\mathbf{2}}\right]-3 \mathbf{y}$ in a $63 \%$ yield as a yellow oil.

The degree of deuteration $\left[\mathbf{D}_{\mathbf{2}}\right]-\mathbf{3 y}$ is almost same as that of vinyl azide $\left[\mathbf{D}_{\mathbf{2}}\right]-\mathbf{6}$. The extent of deuterium incorporation in $\left[\mathbf{D}_{2}\right]-3 \mathbf{y}(\sim 60 \%$ D) was determined from the relative integration of the benzylic protons at $\delta 3.15-3.09(\mathrm{~m}, 0.83 \mathrm{H})$.

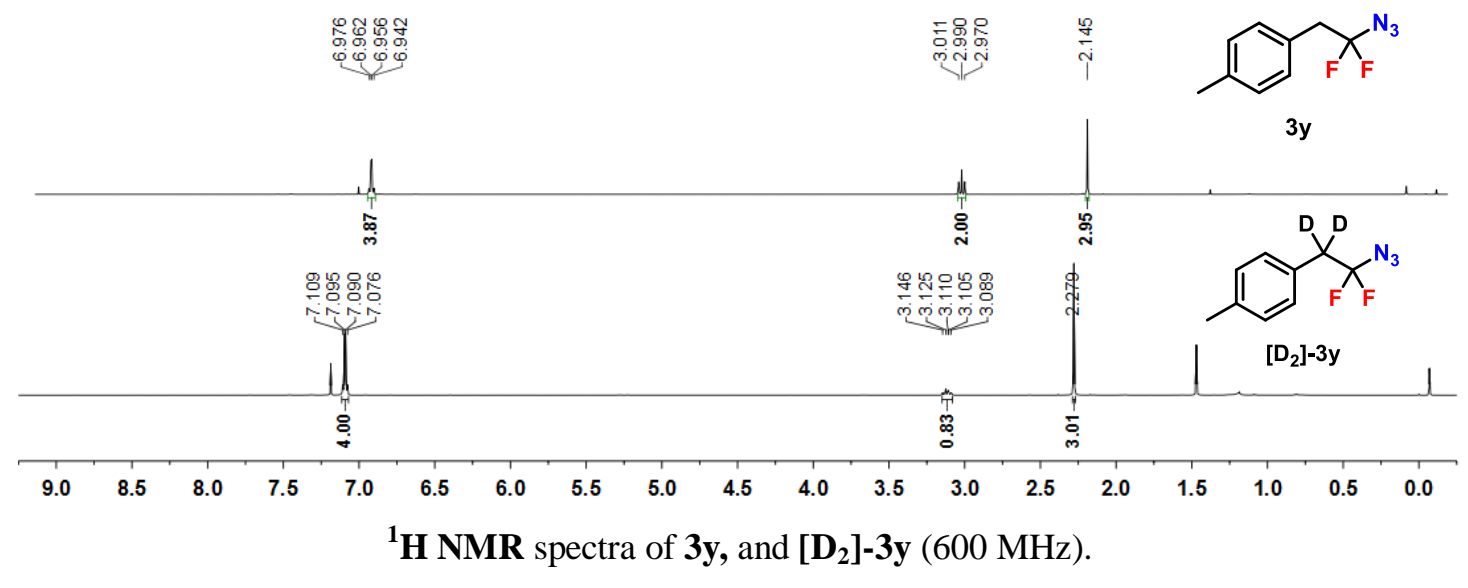

\section{Characterization data for the products}

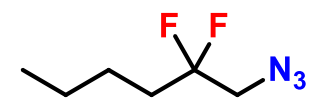

$2 a$

1-azido-2,2-difluorohexane (2a) This compound was prepared according to the general procedure $\mathbf{A}$. The crude mixture was purified by column chromatography with petroleum ether as the eluent to give the product $\mathbf{2 a}$ as a colorless liquid (83.2 mg, $51 \%$ yield). ${ }^{1} \mathbf{H} \mathbf{~ N M R}(600 \mathrm{MHz}$, 
$\left.\mathrm{CDCl}_{3}\right) \delta 3.45(\mathrm{t}, J=12.6 \mathrm{~Hz}, 2 \mathrm{H}), 1.97-1.86(\mathrm{~m}, 2 \mathrm{H}), 1.51-1.42(\mathrm{~m}, 2 \mathrm{H}), 1.41-1.35(\mathrm{~m}, 2 \mathrm{H})$, $0.93(\mathrm{t}, J=7.2 \mathrm{~Hz}, 3 \mathrm{H}) .{ }^{13} \mathbf{C}$ NMR $\left(150 \mathrm{MHz}, \mathrm{CDCl}_{3}\right) \delta 122.9(\mathrm{t}, J=241.5 \mathrm{~Hz}), 53.8(\mathrm{t}, J=31.5$ $\mathrm{Hz}), 33.8(\mathrm{t}, J=22.5 \mathrm{~Hz}), 23.9(\mathrm{t}, J=4.5 \mathrm{~Hz}), 22.4,13.8 .{ }^{19} \mathbf{F}$ NMR $\left(470 \mathrm{MHz}, \mathrm{CDCl}_{3}\right) \delta-102.23$ - $-102.36(\mathrm{~m}) . \mathbf{I R}\left(\mathrm{KBr}, \mathrm{cm}^{-1}\right) 2110$.

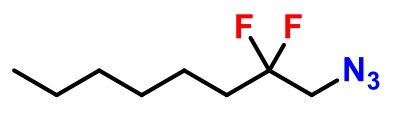

2b

1-azido-2,2-difluorooctane (2b) This compound was prepared according to the general procedure A. The crude mixture was purified by column chromatography with petroleum ether as the eluent to give the product $\mathbf{2 b}$ as a colorless liquid $\left(152.9 \mathrm{mg}, 80 \%\right.$ yield). ${ }^{\mathbf{1}} \mathbf{H} \mathbf{~ N M R}\left(600 \mathrm{MHz}, \mathrm{CDCl}_{3}\right) \delta$ $3.45(\mathrm{t}, J=13.2 \mathrm{~Hz}, 2 \mathrm{H}), 2.00-1.76(\mathrm{~m}, 2 \mathrm{H}), 1.52-1.41(\mathrm{~m}, 2 \mathrm{H}), 1.42-1.21(\mathrm{~m}, 6 \mathrm{H}), 0.89(\mathrm{t}, J=$ $6.6 \mathrm{~Hz}, 3 \mathrm{H}) .{ }^{13} \mathbf{C}$ NMR $\left(150 \mathrm{MHz}, \mathrm{CDCl}_{3}\right) \delta 122.9(\mathrm{t}, J=243.0 \mathrm{~Hz}), 53.8(\mathrm{t}, J=31.5 \mathrm{~Hz}), 34.1(\mathrm{t}$, $J=22.5 \mathrm{~Hz}), 31.5,28.9,22.4,21.8(\mathrm{t}, J=4.5 \mathrm{~Hz}), 14.0 .{ }^{19} \mathbf{F} \mathbf{N M R}\left(470 \mathrm{MHz}, \mathrm{CDCl}_{3}\right) \delta-102.24-$ $-102.36(\mathrm{~m}) . \mathbf{I R}\left(\mathrm{KBr}, \mathrm{cm}^{-1}\right) 2145$.

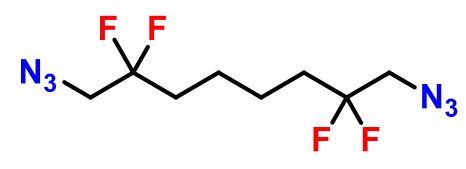

2c

1,8-diazido-2,2,7,7-tetrafluorooctane (2c) This compound was prepared according to the general procedure $\mathbf{A}$. The crude mixture was purified by column chromatography with petroleum ether as the eluent to give the product $\mathbf{2 c}$ as a colorless liquid (211.8 $\mathrm{mg}, 79 \%$ yield). ${ }^{1} \mathbf{H}$ NMR $\left(600 \mathrm{MHz}, \mathrm{CDCl}_{3}\right) \delta 3.46(\mathrm{t}, J=13.2 \mathrm{~Hz}, 4 \mathrm{H}), 1.98-1.90(\mathrm{~m}, 4 \mathrm{H}), 1.61-1.52(\mathrm{~m}, 4 \mathrm{H}) .{ }^{13} \mathrm{C}$ NMR $\left(150 \mathrm{MHz}, \mathrm{CDCl}_{3}\right) \delta 122.6(\mathrm{t}, J=241.5 \mathrm{~Hz}), 53.8(\mathrm{t}, J=30.0 \mathrm{~Hz}), 33.7(\mathrm{t}, J=24.0 \mathrm{~Hz})$, $21.4(\mathrm{t}, J=4.5 \mathrm{~Hz}) .{ }^{19} \mathbf{F}$ NMR $\left(470 \mathrm{MHz}, \mathrm{CDCl}_{3}\right) \delta-103.08--103.21(\mathrm{~m}) . \mathbf{I R}\left(\mathrm{KBr}, \mathrm{cm}^{-1}\right) 2139$.

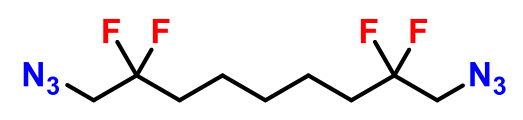

2d

1,9-diazido-2,2,8,8-tetrafluorononane (2d) This compound was prepared according to the general procedure $\mathbf{A}$. The crude mixture was purified by column chromatography with petroleum ether as the eluent to give the product $\mathbf{2 d}$ as a colorless liquid (197.5 mg, 70\% yield). ${ }^{1} \mathbf{H}$ NMR $\left(600 \mathrm{MHz}, \mathrm{CDCl}_{3}\right) \delta 3.46(\mathrm{t}, J=12.6 \mathrm{~Hz}, 4 \mathrm{H}), 1.98-1.87(\mathrm{~m}, 4 \mathrm{H}), 1.56-1.49(\mathrm{~m}, 4 \mathrm{H}), 1.45-$ $1.41(\mathrm{~m}, 2 \mathrm{H}) .{ }^{13} \mathbf{C}$ NMR $\left(150 \mathrm{MHz}, \mathrm{CDCl}_{3}\right) \delta 122.7(\mathrm{t}, J=241.5 \mathrm{~Hz}), 53.9(\mathrm{t}, J=31.5 \mathrm{~Hz}), 33.8$ $(\mathrm{t}, J=24.0 \mathrm{~Hz}), 28.8,21.5(\mathrm{t}, J=4.5 \mathrm{~Hz}) .{ }^{19} \mathbf{F}$ NMR $\left(470 \mathrm{MHz}, \mathrm{CDCl}_{3}\right) \delta-102.48--102.60(\mathrm{~m})$. IR $\left(\mathrm{KBr}, \mathrm{cm}^{-1}\right) 2141$.

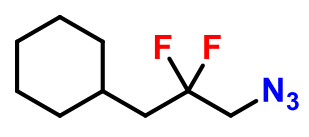


(3-azido-2,2-difluoropropyl)cyclohexane (2e) This compound was prepared according to the general procedure $\mathbf{A}$. The crude mixture was purified by column chromatography with petroleum ether as the eluent to give the product $2 \mathbf{e}$ as a colorless liquid (130.1 mg, 64\% yield). ${ }^{1} \mathbf{H}$ NMR $\left(600 \mathrm{MHz}, \mathrm{CDCl}_{3}\right) \delta 3.43(\mathrm{t}, J=12.6 \mathrm{~Hz}, 2 \mathrm{H}), 1.85-1.76(\mathrm{~m}, 4 \mathrm{H}), 1.73-1.67(\mathrm{~m}, 2 \mathrm{H}), 1.67-$ $1.60(\mathrm{~m}, 2 \mathrm{H}), 1.32$ - $1.23(\mathrm{~m}, 2 \mathrm{H}), 1.19$ - $1.12(\mathrm{~m}, 1 \mathrm{H}), 1.04-0.97(\mathrm{~m}, 2 \mathrm{H}) .{ }^{13} \mathbf{C} \mathbf{N M R}(150 \mathrm{MHz}$, $\left.\mathrm{CDCl}_{3}\right) \delta 123.2(\mathrm{t}, J=243.0 \mathrm{~Hz}), 54.5(\mathrm{t}, J=31.5 \mathrm{~Hz}), 41.2(\mathrm{t}, J=22.5 \mathrm{~Hz}), 33.9,32.1(\mathrm{t}, J=3.0$ $\mathrm{Hz}), 26.07,26.05 .{ }^{19} \mathbf{F}$ NMR $\left(470 \mathrm{MHz}, \mathrm{CDCl}_{3}\right) \delta-99.66$ - $-99.80(\mathrm{~m}) . \mathbf{I R}\left(\mathrm{KBr}, \mathrm{cm}^{-1}\right) 2140$.

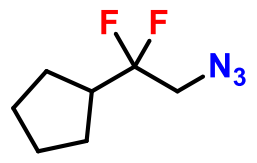

$2 f$

(2-azido-1,1-difluoroethyl)cyclopentane (2f) This compound was prepared according to the general procedure $\mathbf{A}$. The crude mixture was purified by column chromatography with petroleum ether as the eluent to give the product $2 \mathbf{f}$ as a colorless liquid (103.3 mg, 59\% yield). ${ }^{1} \mathbf{H}$ NMR $\left(600 \mathrm{MHz}, \mathrm{CDCl}_{3}\right) \delta 3.47(\mathrm{t}, J=13.2 \mathrm{~Hz}, 2 \mathrm{H}), 2.51-2.37(\mathrm{~m}, 1 \mathrm{H}), 1.83-1.73(\mathrm{~m}, 2 \mathrm{H}), 1.70-$ $1.64(\mathrm{~m}, 2 \mathrm{H}), 1.62-1.57(\mathrm{~m}, 4 \mathrm{H}) .{ }^{13} \mathbf{C}$ NMR $\left(150 \mathrm{MHz}, \mathrm{CDCl}_{3}\right) \delta 123.8(\mathrm{t}, J=243.0 \mathrm{~Hz}), 53.7(\mathrm{t}$, $J=30.0 \mathrm{~Hz}), 42.9(\mathrm{t}, J=22.5 \mathrm{~Hz}), 25.9(\mathrm{t}, J=4.5 \mathrm{~Hz}), 25.8 .{ }^{19} \mathbf{F} \mathbf{N M R}\left(470 \mathrm{MHz}, \mathrm{CDCl}_{3}\right) \delta$ $-108.89(\mathrm{dt}, J=15.0,13.8 \mathrm{~Hz}) . \mathbf{I R}\left(\mathrm{KBr}, \mathrm{cm}^{-1}\right) 2145$.<smiles>NCC(F)(F)C1CCCCC1</smiles>

\section{$2 \mathrm{~g}$}

(2-azido-1,1-difluoroethyl)cyclohexane (2g) This compound was prepared according to the general procedure $\mathbf{A}$. The crude mixture was purified by column chromatography with petroleum ether as the eluent to give the product $\mathbf{2 g}$ as a colorless liquid (123.0 $\mathrm{mg}, 65 \%$ yield). ${ }^{\mathbf{1}} \mathbf{H}$ NMR $\left(600 \mathrm{MHz}, \mathrm{CDCl}_{3}\right) \delta 3.47(\mathrm{t}, J=13.8 \mathrm{~Hz}, 2 \mathrm{H}), 1.97-1.86(\mathrm{~m}, 1 \mathrm{H}), 1.85-1.79(\mathrm{~m}, 4 \mathrm{H}), 1.71-$ $1.69(\mathrm{~m}, 1 \mathrm{H}), 1.30-1.11(\mathrm{~m}, 5 \mathrm{H}) .{ }^{13} \mathbf{C}$ NMR $\left(150 \mathrm{MHz}, \mathrm{CDCl}_{3}\right) \delta 123.6(\mathrm{t}, J=244.5 \mathrm{~Hz}), 52.2(\mathrm{t}$, $J=31.5 \mathrm{~Hz}), 41.7(\mathrm{t}, J=21.0 \mathrm{~Hz}), 25.8,25.5,25.3(\mathrm{t}, J=3.0 \mathrm{~Hz}) .{ }^{19} \mathbf{F} \mathbf{N M R}\left(470 \mathrm{MHz}, \mathrm{CDCl}_{3}\right)$ $\delta-109.20(\mathrm{q}, J=14.1 \mathrm{~Hz})$. IR $\left(\mathrm{KBr}, \mathrm{cm}^{-1}\right) 2143$.<smiles>NCC(F)(F)Cc1ccccc1</smiles>

$2 h$

(3-azido-2,2-difluoropropyl)benzene (2h) This compound was prepared according to the general procedure $\mathbf{A}$. The crude mixture was purified by column chromatography with petroleum ether as the eluent to give the product $\mathbf{2 h}$ as a colorless liquid (157.7 mg, 80\% yield). ${ }^{1} \mathbf{H} \mathbf{~ N M R}(600 \mathrm{MHz}$, $\left.\mathrm{CDCl}_{3}\right) \delta 7.35-7.27(\mathrm{~m}, 3 \mathrm{H}), 7.26(\mathrm{~d}, J=7.2 \mathrm{~Hz}, 2 \mathrm{H}), 3.34(\mathrm{t}, J=12.6 \mathrm{~Hz}, 2 \mathrm{H}), 3.22(\mathrm{t}, J=15.6$ $\mathrm{Hz}, 2 \mathrm{H}) .{ }^{13} \mathbf{C}$ NMR $\left(150 \mathrm{MHz}, \mathrm{CDCl}_{3}\right) \delta 132.1(\mathrm{t}, J=4.5 \mathrm{~Hz}), 130.3,128.6,127.7,122.0(\mathrm{t}, J=$ $244.5 \mathrm{~Hz}), 52.8(\mathrm{t}, J=31.5 \mathrm{~Hz}), 40.4(\mathrm{t}, J=24.0 \mathrm{~Hz}) .{ }^{19} \mathbf{F} \mathbf{N M R}\left(470 \mathrm{MHz}, \mathrm{CDCl}_{3}\right) \delta-101.03-$ $-101.15(\mathrm{~m}) . \mathbf{I R}\left(\mathrm{KBr}, \mathrm{cm}^{-1}\right) 2146$. 
<smiles>NCC(F)(F)CCc1ccccc1</smiles>

2i

(4-azido-3,3-difluorobutyl)benzene (2i) This compound was prepared according to the general procedure $\mathbf{A}$. The crude mixture was purified by column chromatography with petroleum ether as the eluent to give the product $\mathbf{2 i}$ as a colorless liquid (137.3 mg, 65\% yield). ${ }^{1} \mathbf{H} \mathbf{~ N M R}$ (600 MHz, $\left.\mathrm{CDCl}_{3}\right) \delta 7.30(\mathrm{t}, J=7.2 \mathrm{~Hz}, 2 \mathrm{H}), 7.24-7.18(\mathrm{~m}, 3 \mathrm{H}), 3.46(\mathrm{t}, J=12.6 \mathrm{~Hz}, 2 \mathrm{H}), 2.84-2.79(\mathrm{~m}$, 2H), 2.28 - 2.17 (m, 2H). ${ }^{13} \mathbf{C ~ N M R ~}\left(150 \mathrm{MHz}, \mathrm{CDCl}_{3}\right) \delta 139.9,128.6,128.2,126.4,122.3(\mathrm{t}, J=$ $243.0 \mathrm{~Hz}), 53.9(\mathrm{t}, J=31.5 \mathrm{~Hz}), 35.9(\mathrm{t}, J=24.0 \mathrm{~Hz}), 27.9(\mathrm{t}, J=4.5 \mathrm{~Hz}) .{ }^{19} \mathbf{F}$ NMR $(470 \mathrm{MHz}$, $\left.\mathrm{CDCl}_{3}\right) \delta-102.81--102.94(\mathrm{~m}) . \mathbf{I R}\left(\mathrm{KBr}, \mathrm{cm}^{-1}\right) 2148$.<smiles>NCC(F)(F)CCCc1ccccc1</smiles>

2j

(5-azido-4,4-difluoropentyl)benzene (2j) This compound was prepared according to the general procedure $\mathbf{A}$. The crude mixture was purified by column chromatography with petroleum ether as the eluent to give the product $\mathbf{2} \mathbf{j}$ as a colorless liquid (191.4 $\mathrm{mg}, 85 \%$ yield). ${ }^{\mathbf{1}} \mathbf{H}$ NMR (600 $\left.\mathrm{MHz}, \mathrm{CDCl}_{3}\right) \delta 7.29$ (t, $\left.J=7.8 \mathrm{~Hz}, 2 \mathrm{H}\right), 7.22-7.17(\mathrm{~m}, 3 \mathrm{H}), 3.43(\mathrm{t}, J=12.6 \mathrm{~Hz}, 2 \mathrm{H}), 2.67(\mathrm{t}, J$ $=7.8 \mathrm{~Hz}, 2 \mathrm{H}), 2.00-1.87(\mathrm{~m}, 2 \mathrm{H}), 1.87-1.79(\mathrm{~m}, 2 \mathrm{H}) .{ }^{13} \mathbf{C} \mathbf{~ N M R}\left(150 \mathrm{MHz}, \mathrm{CDCl}_{3}\right) \delta 141.1$, 128.5, 128.4, 126.1, 122.8 (t, $J=241.5 \mathrm{~Hz}), 53.9$ (t, $J=31.5 \mathrm{~Hz}), 35.2,33.5$ (t, $J=24.0 \mathrm{~Hz}$ ), $23.4(\mathrm{t}, J=4.5 \mathrm{~Hz}) .{ }^{19} \mathbf{F}$ NMR $\left(470 \mathrm{MHz}, \mathrm{CDCl}_{3}\right) \delta-102.37--102.49(\mathrm{~m}) . \mathbf{I R}\left(\mathrm{KBr}, \mathrm{cm}^{-1}\right) 2147$.<smiles>NCC(F)(F)CCCCl</smiles>

2k

1-azido-5-chloro-2,2-difluoropentane (2k) This compound was prepared according to the general procedure $\mathbf{A}$. The crude mixture was purified by column chromatography with petroleum ether as the eluent to give the product $\mathbf{2} \mathbf{k}$ as a colorless liquid (140.9 $\mathrm{mg}, 77 \%$ yield). ${ }^{1} \mathbf{H}$ NMR $\left(600 \mathrm{MHz}, \mathrm{CDCl}_{3}\right) \delta 3.53(\mathrm{t}, J=6.6 \mathrm{~Hz}, 2 \mathrm{H}), 3.42(\mathrm{t}, J=13.2 \mathrm{~Hz}, 2 \mathrm{H}), 2.08-1.98(\mathrm{~m}, 2 \mathrm{H}), 1.97$ - $1.91(\mathrm{~m}, 2 \mathrm{H}) .{ }^{13} \mathrm{C}$ NMR $\left(150 \mathrm{MHz}, \mathrm{CDCl}_{3}\right) \delta 122.4(\mathrm{t}, J=243.0 \mathrm{~Hz}), 54.1(\mathrm{t}, J=30.0 \mathrm{~Hz})$, $44.0,31.5(\mathrm{t}, J=24.0 \mathrm{~Hz}), 24.9(\mathrm{t}, J=4.5 \mathrm{~Hz}) .{ }^{19} \mathbf{F} \mathbf{N M R}\left(470 \mathrm{MHz}, \mathrm{CDCl}_{3}\right) \delta-103.02--103.15$ (m). IR $\left(\mathrm{KBr}, \mathrm{cm}^{-1}\right) 2114$.

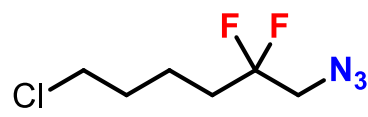

2I

1-azido-6-chloro-2,2-difluorohexane (2l) This compound was prepared according to the general procedure $\mathbf{A}$. The crude mixture was purified by column chromatography with petroleum ether as the eluent to give the product $\mathbf{2} \mathbf{l}$ as a colorless liquid $\left(159.7 \mathrm{mg}, 81 \%\right.$ yield). ${ }^{\mathbf{1}} \mathbf{H}$ NMR (600 $\left.\mathrm{MHz}, \mathrm{CDCl}_{3}\right) \delta 3.56(\mathrm{t}, J=6.0 \mathrm{~Hz}, 2 \mathrm{H}), 3.47(\mathrm{t}, J=13.2 \mathrm{~Hz}, 2 \mathrm{H}), 2.00-1.90(\mathrm{~m}, 2 \mathrm{H}), 1.87-$ $1.81(\mathrm{~m}, 2 \mathrm{H}), 1.69-1.64(\mathrm{~m}, 2 \mathrm{H}) .{ }^{13} \mathbf{C}$ NMR $\left(150 \mathrm{MHz}, \mathrm{CDCl}_{3}\right) \delta 122.6(\mathrm{t}, J=241.5 \mathrm{~Hz}), 53.8(\mathrm{t}$, 
$J=31.5 \mathrm{~Hz}), 44.3,33.3(\mathrm{t}, J=24.0 \mathrm{~Hz}), 31.9,19.2(\mathrm{t}, J=4.5 \mathrm{~Hz}) .{ }^{19} \mathbf{F} \mathbf{N M R}\left(470 \mathrm{MHz}, \mathrm{CDCl}_{3}\right)$ $\delta$-102.53 - -102.66(m). IR $\left(\mathrm{KBr}, \mathrm{cm}^{-1}\right) 2117$.

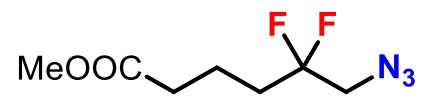

\section{$2 m$}

methyl 6-azido-5,5-difluorohexanoate (2m) This compound was prepared according to the general procedure $\mathbf{A}$. The crude mixture was purified by column chromatography with petroleum ether as the eluent to give the product $\mathbf{2} \mathbf{m}$ as a colorless liquid (157.4 $\mathrm{mg}, 76 \%$ yield). ${ }^{\mathbf{1}} \mathbf{H}$ NMR $\left(600 \mathrm{MHz}, \mathrm{CDCl}_{3}\right) \delta 3.69(\mathrm{~s}, 3 \mathrm{H}), 3.48(\mathrm{t}, J=13.2 \mathrm{~Hz}, 2 \mathrm{H}), 2.40(\mathrm{t}, J=7.2 \mathrm{~Hz}, 2 \mathrm{H}), 2.02-1.93$ $(\mathrm{m}, 2 \mathrm{H}), 1.87-1.82(\mathrm{~m}, 2 \mathrm{H}) .{ }^{13} \mathbf{C}$ NMR $\left(150 \mathrm{MHz}, \mathrm{CDCl}_{3}\right) \delta 173.1,122.5(\mathrm{t}, J=243.0 \mathrm{~Hz}), 53.8$ $(\mathrm{t}, J=30.0 \mathrm{~Hz}), 51.6,33.2(\mathrm{t}, J=24.0 \mathrm{~Hz}), 33.1,17.2(\mathrm{t}, J=4.5 \mathrm{~Hz}) .{ }^{19} \mathbf{F}$ NMR $(470 \mathrm{MHz}$, $\left.\mathrm{CDCl}_{3}\right) \delta-102.86--102.98(\mathrm{~m}) . \mathbf{I R}\left(\mathrm{KBr}, \mathrm{cm}^{-1}\right) 2154$.

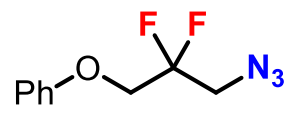

2n

(3-azido-2,2-difluoropropoxy)benzene (2n) This compound was prepared according to the general procedure $\mathbf{A}$. The crude mixture was purified by column chromatography with petroleum ether as the eluent to give the product $\mathbf{2 n}$ as a colorless liquid (149.2 $\mathrm{mg}, 70 \%$ yield). ${ }^{\mathbf{1}} \mathbf{H}$ NMR $\left(600 \mathrm{MHz}, \mathrm{CDCl}_{3}\right) \delta 7.32(\mathrm{t}, J=7.8 \mathrm{~Hz}, 2 \mathrm{H}), 7.03(\mathrm{t}, J=7.2 \mathrm{~Hz}, 1 \mathrm{H}), 6.93(\mathrm{~d}, J=7.8 \mathrm{~Hz}, 2 \mathrm{H})$, $4.24(\mathrm{t}, J=11.4 \mathrm{~Hz}, 2 \mathrm{H}), 3.75(\mathrm{t}, J=13.2 \mathrm{~Hz}, 2 \mathrm{H}) .{ }^{13} \mathbf{C} \mathbf{N M R}\left(150 \mathrm{MHz}, \mathrm{CDCl}_{3}\right) \delta 157.5,129.7$, 122.2, $120.1(\mathrm{t}, J=243.0 \mathrm{~Hz}), 114.7,66.3(\mathrm{t}, J=33.0 \mathrm{~Hz}), 51.5(\mathrm{t}, J=30.0 \mathrm{~Hz}) .{ }^{19} \mathbf{F}$ NMR $(470$ $\left.\mathrm{MHz}, \mathrm{CDCl}_{3}\right) \delta-109.79$ - -109.90 (m). IR $\left(\mathrm{KBr}, \mathrm{cm}^{-1}\right) 2144$.<smiles>NCC(F)(F)COC(c1ccccc1)c1ccccc1</smiles>

20

((3-azido-2,2-difluoropropoxy)methylene)dibenzene (20) This compound was prepared according to the general procedure A. The crude mixture was purified by column chromatography with petroleum ether as the eluent to give the product $\mathbf{2 0}$ as a yellow liquid (221.3 mg, 73\% yield). ${ }^{1} \mathbf{H}$ NMR $\left(600 \mathrm{MHz}, \mathrm{CDCl}_{3}\right) \delta 7.35-7.30(\mathrm{~m}, 8 \mathrm{H}), 7.29$ - $7.24(\mathrm{~m}, 2 \mathrm{H})$, $5.45(\mathrm{~s}, 1 \mathrm{H}), 3.71(\mathrm{t}, J=12.0 \mathrm{~Hz}, 2 \mathrm{H}), 3.65(\mathrm{t}, J=13.2 \mathrm{~Hz}, 2 \mathrm{H}) .{ }^{13} \mathbf{C ~ N M R}\left(150 \mathrm{MHz}, \mathrm{CDCl}_{3}\right) \delta$ $140.8,128.5,127.9,126.9,120.7(\mathrm{t}, J=243.0 \mathrm{~Hz}), 84.6,67.2(\mathrm{t}, J=31.5 \mathrm{~Hz}), 51.5(\mathrm{t}, J=28.5$ Hz). ${ }^{19}$ F NMR $\left(470 \mathrm{MHz}, \mathrm{CDCl}_{3}\right) \delta-109.64$ - -109.75 (m). IR (KBr, $\left.\mathrm{cm}^{-1}\right) 2147$.<smiles>N#Cc1ccc(C(F)(F)CN)cc1</smiles>

4-(2-azido-1,1-difluoroethyl)benzonitrile (2p) This compound was prepared according to the general procedure $\mathbf{A}$. The crude mixture was purified by column chromatography with petroleum ether as the eluent to give the product $\mathbf{2} \mathbf{p}$ as a colorless liquid (124.9 $\mathrm{mg}, 60 \%$ yield). ${ }^{1} \mathbf{H}$ NMR 
$\left(600 \mathrm{MHz}, \mathrm{CDCl}_{3}\right) \delta 7.78(\mathrm{~d}, J=8.4 \mathrm{~Hz}, 2 \mathrm{H}), 7.65(\mathrm{~d}, J=8.4 \mathrm{~Hz}, 2 \mathrm{H}), 3.73(\mathrm{t}, J=12.6 \mathrm{~Hz}, 2 \mathrm{H})$. ${ }^{13} \mathbf{C ~ N M R}\left(150 \mathrm{MHz}, \mathrm{CDCl}_{3}\right) \delta 138.6(\mathrm{t}, J=25.5 \mathrm{~Hz}), 132.5,126.3(\mathrm{t}, J=6.0 \mathrm{~Hz}), 119.3(\mathrm{t}, J=$ $246.0 \mathrm{~Hz}), 117.7,114.9(\mathrm{t}, J=1.5 \mathrm{~Hz}), 55.7(\mathrm{t}, J=31.5 \mathrm{~Hz}) .{ }^{19} \mathbf{F}$ NMR $\left(470 \mathrm{MHz}, \mathrm{CDCl}_{3}\right) \delta$ $-101.86(\mathrm{t}, J=12.6 \mathrm{~Hz}) . \mathbf{I R}\left(\mathrm{KBr}, \mathrm{cm}^{-1}\right) 2143$.<smiles>NCC(F)(F)c1ccc([N+](=O)[O-])cc1</smiles>

$2 q$

1-(2-azido-1,1-difluoroethyl)-4-nitrobenzene (2q) This compound was prepared according to the general procedure A. The crude mixture was purified by column chromatography with petroleum ether as the eluent to give the product $\mathbf{2 q}$ as a colorless liquid $(116.3 \mathrm{mg}, 51 \%$ yield). ${ }^{1} \mathbf{H}$ NMR $\left(600 \mathrm{MHz}, \mathrm{CDCl}_{3}\right) \delta 8.34(\mathrm{~d}, J=8.4 \mathrm{~Hz}, 2 \mathrm{H}), 7.72(\mathrm{~d}, J=8.4 \mathrm{~Hz}, 2 \mathrm{H}), 3.77(\mathrm{t}, J$ $=12.6 \mathrm{~Hz}, 2 \mathrm{H}) .{ }^{13} \mathbf{C}$ NMR $\left(150 \mathrm{MHz}, \mathrm{CDCl}_{3}\right) \delta 149.3,140.3(\mathrm{t}, J=25.5 \mathrm{~Hz}), 126.8(\mathrm{t}, J=6.0$ $\mathrm{Hz}), 123.9,119.3(\mathrm{t}, J=246.0 \mathrm{~Hz}), 55.8(\mathrm{t}, J=31.5 \mathrm{~Hz}) .{ }^{19} \mathbf{F} \mathbf{N M R}\left(470 \mathrm{MHz}, \mathrm{CDCl}_{3}\right) \delta-101.40$ $(\mathrm{t}, J=12.6 \mathrm{~Hz})$. IR $\left(\mathrm{KBr}, \mathrm{cm}^{-1}\right) 2148$.<smiles>CS(=O)(=O)c1ccc(C(F)(F)CN)cc1</smiles>

$2 r$

1-(2-azido-1,1-difluoroethyl)-4-(methylsulfonyl)benzene (2r) This compound was prepared according to the general procedure A. The crude mixture was purified by column chromatography with petroleum ether as the eluent to give the product $\mathbf{2 r}$ as a colorless solid (143.6 mg, 55\% yield); m.p. $125-126{ }^{\circ} \mathrm{C} ;{ }^{1} \mathbf{H}$ NMR $\left(600 \mathrm{MHz}, \mathrm{CDCl}_{3}\right) \delta 8.07$ (d, J=8.4 Hz, 2H), $7.74(\mathrm{~d}, J=8.4 \mathrm{~Hz}, 2 \mathrm{H}), 3.76(\mathrm{t}, J=12.6 \mathrm{~Hz}, 2 \mathrm{H}), 3.09$ (s, 3H). ${ }^{13} \mathbf{C}$ NMR $(150 \mathrm{MHz}$, $\left.\mathrm{CDCl}_{3}\right) \delta 142.8,139.5(\mathrm{t}, J=27.0 \mathrm{~Hz}), 127.9,126.7(\mathrm{t}, J=6.0 \mathrm{~Hz}), 119.4(\mathrm{t}, J=244.5 \mathrm{~Hz}), 55.7$ $(\mathrm{t}, J=31.5 \mathrm{~Hz}), 44.4 .{ }^{19} \mathbf{F}$ NMR $\left(470 \mathrm{MHz}, \mathrm{CDCl}_{3}\right) \delta-101.54(\mathrm{t}, J=12.6 \mathrm{~Hz}) . \mathbf{I R}\left(\mathrm{KBr}, \mathrm{cm}^{-1}\right)$ 2145 .

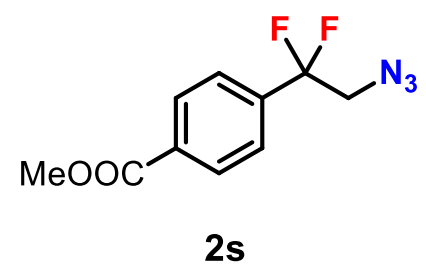

methyl 4-(2-azido-1,1-difluoroethyl)benzoate (2s) This compound was prepared according to the general procedure A. The crude mixture was purified by column chromatography with petroleum ether as the eluent to give the product $2 \mathrm{~s}$ as a colorless liquid (123.9 $\mathrm{mg}, 51 \%$ yield). ${ }^{1} \mathbf{H}$ NMR $\left(600 \mathrm{MHz}, \mathrm{CDCl}_{3}\right) \delta 8.13(\mathrm{~d}, J=8.4 \mathrm{~Hz}, 2 \mathrm{H}), 7.59(\mathrm{~d}, J=8.4 \mathrm{~Hz}, 2 \mathrm{H}), 3.95(\mathrm{~s}, 3 \mathrm{H})$, $3.72(\mathrm{t}, J=13.2 \mathrm{~Hz}, 2 \mathrm{H}) .{ }^{13} \mathrm{C}$ NMR $\left(150 \mathrm{MHz}, \mathrm{CDCl}_{3}\right) \delta 166.1,138.4(\mathrm{t}, J=25.5 \mathrm{~Hz}), 132.3$, 129.9, $125.5(\mathrm{t}, J=6.0 \mathrm{~Hz}), 119.8(\mathrm{t}, J=244.5 \mathrm{~Hz}), 55.9(\mathrm{t}, J=31.5 \mathrm{~Hz}), 52.4 .{ }^{19} \mathbf{F}$ NMR $(470$ $\left.\mathrm{MHz}, \mathrm{CDCl}_{3}\right) \delta-101.71(\mathrm{t}, J=13.2 \mathrm{~Hz})$. IR $\left(\mathrm{KBr}, \mathrm{cm}^{-1}\right) 2146$. 
<smiles>COC(=O)c1cccc(C(F)(F)CN)c1</smiles>

$2 t$

methyl 3-(2-azido-1,1-difluoroethyl)benzoate (2t) This compound was prepared according to the general procedure A. The crude mixture was purified by column chromatography with petroleum ether as the eluent to give the product $\mathbf{2 t}$ as a colorless liquid (95.3 $\mathrm{mg}, 40 \%$ yield). ${ }^{1} \mathbf{H}$ NMR $\left(600 \mathrm{MHz}, \mathrm{CDCl}_{3}\right) \delta 8.21-8.15(\mathrm{~m}, 2 \mathrm{H}), 7.72(\mathrm{~d}, J=7.8 \mathrm{~Hz}, 1 \mathrm{H}), 7.56(\mathrm{t}, J=7.8 \mathrm{~Hz}, 1 \mathrm{H})$, $3.95(\mathrm{~s}, 3 \mathrm{H}), 3.74(\mathrm{t}, J=13.2 \mathrm{~Hz}, 2 \mathrm{H}) .{ }^{13} \mathbf{C ~ N M R}\left(150 \mathrm{MHz}, \mathrm{CDCl}_{3}\right) \delta 166.1,134.7(\mathrm{t}, J=25.5$ $\mathrm{Hz}), 131.7,130.9,129.7(\mathrm{t}, J=6.0 \mathrm{~Hz}), 129.0,126.6(\mathrm{t}, J=7.5 \mathrm{~Hz}), 119.8(\mathrm{t}, J=244.5 \mathrm{~Hz}), 55.9$ $(\mathrm{t}, J=33.0 \mathrm{~Hz}), 52.4 .{ }^{19} \mathbf{F}$ NMR $\left(470 \mathrm{MHz} \mathrm{CDCl}_{3}\right) \delta-101.16(\mathrm{t}, J=13.2 \mathrm{~Hz}) . \mathbf{I R}\left(\mathrm{KBr}, \mathrm{cm}^{-1}\right)$ 2148 .<smiles>NCC(F)(F)c1ccc(C=O)cc1</smiles>

$2 \mathbf{u}$

4-(2-azido-1,1-difluoroethyl)benzaldehyde (2u) This compound was prepared according to the general procedure $\mathbf{A}$. The crude mixture was purified by column chromatography with petroleum ether as the eluent to give the product $\mathbf{2 u}$ as a colorless solid (95.6 mg, $45 \%$ yield). m.p. 95 - 96 ${ }^{\circ} \mathrm{C} ;{ }^{1} \mathbf{H}$ NMR $\left(600 \mathrm{MHz}, \mathrm{CDCl}_{3}\right) \delta 10.09(\mathrm{~s}, 1 \mathrm{H}), 7.99(\mathrm{~d}, J=8.0 \mathrm{~Hz}, 2 \mathrm{H}), 7.70(\mathrm{~d}, J=8.0 \mathrm{~Hz}$, $2 \mathrm{H}), 3.74(\mathrm{t}, J=12.6 \mathrm{~Hz}, 2 \mathrm{H}) .{ }^{13} \mathbf{C} \mathbf{N M R}\left(150 \mathrm{MHz}, \mathrm{CDCl}_{3}\right) \delta 190.3,138.8(\mathrm{t}, J=23.5 \mathrm{~Hz})$, 136.8, 128.9, $125.2(\mathrm{t}, J=6.0 \mathrm{~Hz}), 118.7(\mathrm{t}, J=244.5 \mathrm{~Hz}), 54.8(\mathrm{t}, J=33.0 \mathrm{~Hz}) .{ }^{19} \mathbf{F} \mathbf{N M R}(470$ $\left.\mathrm{MHz} \mathrm{CDCl}_{3}\right) \delta-101.69(\mathrm{t}, J=12.6 \mathrm{~Hz}) . \mathbf{I R}\left(\mathrm{KBr}, \mathrm{cm}^{-1}\right) 2142$.<smiles>COC(=O)c1ccc(CC([NH-])(F)F)cc1</smiles>

$3 s$

methyl 4-(2-azido-2,2-difluoroethyl)benzoate (3s) This compound was prepared according to the general procedure $\mathbf{A}$. The crude mixture was purified by column chromatography with petroleum ether as the eluent to give the product $\mathbf{3 s}$ as a colorless liquid (32.8 $\mathrm{mg}, 14 \%$ yield). ${ }^{1} \mathbf{H}$ NMR $\left(600 \mathrm{MHz}, \mathrm{CDCl}_{3}\right) \delta 8.02(\mathrm{~d}, J=8.4 \mathrm{~Hz}, 2 \mathrm{H}), 7.36(\mathrm{~d}, J=8.4 \mathrm{~Hz}, 2 \mathrm{H}), 3.92(\mathrm{~s}, 3 \mathrm{H}), 3.29$ (t, $J=12.6 \mathrm{~Hz}, 2 \mathrm{H}) .{ }^{13} \mathrm{C}$ NMR $\left(150 \mathrm{MHz}, \mathrm{CDCl}_{3}\right) \delta 166.7,136.1,130.5,129.9,129.7,122.6(\mathrm{t}, J=$ $265.5 \mathrm{~Hz}), 52.2,42.1(\mathrm{t}, J=27.0 \mathrm{~Hz}) .{ }^{19} \mathbf{F ~ N M R}\left(470 \mathrm{MHz}, \mathrm{CDCl}_{3}\right) \delta-72.11(\mathrm{t}, J=12.6 \mathrm{~Hz}) . \mathbf{I R}$ $\left(\mathrm{KBr}, \mathrm{cm}^{-1}\right) 2110$.<smiles>COC(OC)c1cccc(CC(N)(F)F)c1</smiles> 
methyl 3-(2-azido-2,2-difluoroethyl)benzoate (3t) This compound was prepared according to the general procedure $\mathbf{A}$. The crude mixture was purified by column chromatography with petroleum ether as the eluent to give the product $\mathbf{3 t}$ as a colorless liquid $\left(71.1 \mathrm{mg}, 29 \%\right.$ yield). ${ }^{\mathbf{1}} \mathbf{H}$ NMR (600 $\left.\mathrm{MHz}, \mathrm{CDCl}_{3}\right) \delta 8.04-7.99(\mathrm{~m}, 1 \mathrm{H}), 7.97(\mathrm{~s}, 1 \mathrm{H}), 7.49(\mathrm{~d}, J=7.8 \mathrm{~Hz}, 1 \mathrm{H}), 7.43(\mathrm{t}, J=7.2 \mathrm{~Hz}, 1 \mathrm{H})$, $3.93(\mathrm{~s}, 3 \mathrm{H}), 3.28(\mathrm{t}, J=12.2 \mathrm{~Hz}, 2 \mathrm{H}) .{ }^{13} \mathbf{C}$ NMR $\left(150 \mathrm{MHz}, \mathrm{CDCl}_{3}\right) \delta 166.7,134.9,131.6,131.5$ (t, $J=3.0 \mathrm{~Hz}), 130.5,129.2,128.6,122.7(\mathrm{t}, J=265.5 \mathrm{~Hz}), 52.2,42.0(\mathrm{t}, J=27.0 \mathrm{~Hz}) .{ }^{19} \mathbf{F}$ NMR $\left(470 \mathrm{MHz}, \mathrm{CDCl}_{3}\right) \delta-72.46(\mathrm{t}, J=12.2 \mathrm{~Hz}) . \mathbf{I R}\left(\mathrm{KBr}, \mathrm{cm}^{-1}\right) 2109$.<smiles>NC(F)(F)Cc1ccc(C=O)cc1</smiles>

$3 u$

4-(2-azido-2,2-difluoroethyl)benzaldehyde (3u) This compound was prepared according to the general procedure $\mathbf{A}$. The crude mixture was purified by column chromatography with petroleum ether as the eluent to give the product $3 \mathbf{u}$ as a colorless solid (26.8 mg, 13\% yield). m.p. $98-99$ ${ }^{\circ} \mathrm{C} ;{ }^{1} \mathbf{H}$ NMR $\left(600 \mathrm{MHz}, \mathrm{CDCl}_{3}\right) \delta 10.03(\mathrm{~s}, 1 \mathrm{H}), 7.88(\mathrm{~d}, J=7.8 \mathrm{~Hz}, 2 \mathrm{H}), 7.47(\mathrm{~d}, J=7.8 \mathrm{~Hz}, 2 \mathrm{H})$, $3.32(\mathrm{t}, J=12.6 \mathrm{~Hz}, 2 \mathrm{H}) .{ }^{13} \mathbf{C}$ NMR $\left(150 \mathrm{MHz}, \mathrm{CDCl}_{3}\right) \delta 191.7,137.8(\mathrm{t}, J=3.0 \mathrm{~Hz}), 136.0$, $131.2,129.8,122.6(\mathrm{t}, J=264.0 \mathrm{~Hz}), 42.3(\mathrm{t}, J=28.5 \mathrm{~Hz}) .{ }^{\mathbf{1}} \mathbf{F} \mathbf{~ N M R}\left(470 \mathrm{MHz}, \mathrm{CDCl}_{3}\right) \delta-71.93$ $(\mathrm{t}, J=12.6 \mathrm{~Hz}) . \mathbf{I R}\left(\mathrm{KBr}, \mathrm{cm}^{-1}\right) 2110$.

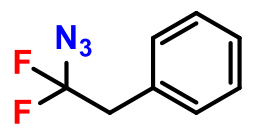

$3 v$

(2-azido-2,2-difluoroethyl)benzene (3v) This compound was prepared according to the general procedure A. The crude mixture was purified by column chromatography with petroleum ether as the eluent to give the product $3 \mathbf{v}$ as a yellow liquid (113.5 mg, $62 \%$ yield). ${ }^{1} \mathbf{H}$ NMR (600 MHz, $\left.\mathrm{CDCl}_{3}\right) \delta 7.37$ - $7.32(\mathrm{~m}, 3 \mathrm{H}), 7.31-7.26(\mathrm{~m}, 2 \mathrm{H}), 3.23(\mathrm{t}, J=12.6 \mathrm{~Hz}, 2 \mathrm{H}) .{ }^{13} \mathbf{C}$ NMR $(150 \mathrm{MHz}$, $\left.\mathrm{CDCl}_{3}\right) \delta 131.1(\mathrm{t}, J=3.0 \mathrm{~Hz}), 130.4,128.5,128.0,122.9(\mathrm{t}, J=265.5 \mathrm{~Hz}), 42.2(\mathrm{t}, J=27.0 \mathrm{~Hz})$. ${ }^{19}$ F NMR $\left(470 \mathrm{MHz}, \mathrm{CDCl}_{3}\right) \delta-72.56(\mathrm{t}, J=12.6 \mathrm{~Hz}) . \mathbf{I R}\left(\mathrm{KBr}, \mathrm{cm}^{-1}\right) 2110$.

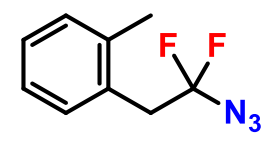

$3 w$

1-(2-azido-2,2-difluoroethyl)-2-methylbenzene (3w) This compound was prepared according to the general procedure A. The crude mixture was purified by column chromatography with petroleum ether as the eluent to give the product $\mathbf{3 w}$ as a yellow liquid (104.5 mg, 53\% yield). ${ }^{1} \mathbf{H}$ NMR $\left(600 \mathrm{MHz}, \mathrm{CDCl}_{3}\right) \delta 7.26-7.16(\mathrm{~m}, 4 \mathrm{H}), 3.27(\mathrm{t}, J=12.6 \mathrm{~Hz}, 2 \mathrm{H}), 2.36(\mathrm{~s}, 3 \mathrm{H}) .{ }^{13} \mathbf{C} \mathbf{N M R}$ $\left(150 \mathrm{MHz}, \mathrm{CDCl}_{3}\right) \delta 137.7,131.5,130.6,129.5(\mathrm{t}, J=3.0 \mathrm{~Hz}), 128.1,126.0,123.4$ (t, $J=265.5$ $\mathrm{Hz}), 38.9(\mathrm{t}, J=27.0 \mathrm{~Hz}), 19.8 .{ }^{19} \mathbf{F}$ NMR $\left(470 \mathrm{MHz}, \mathrm{CDCl}_{3}\right) \delta-71.81(\mathrm{t}, J=12.6 \mathrm{~Hz})$. IR $(\mathrm{KBr}$, $\left.\mathrm{cm}^{-1}\right) 2109$. 


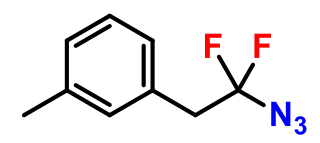

$3 x$

1-(2-azido-2,2-difluoroethyl)-3-methylbenzene (3x) This compound was prepared according to the general procedure A. The crude mixture was purified by column chromatography with petroleum ether as the eluent to give the product $\mathbf{3 x}$ as a yellow liquid (132.1 $\mathrm{mg}, 67 \%$ yield). ${ }^{1} \mathbf{H}$ $\operatorname{NMR}\left(600 \mathrm{MHz}, \mathrm{CDCl}_{3}\right) \delta 7.24(\mathrm{~d}, J=7.8 \mathrm{~Hz}, 1 \mathrm{H}), 7.14(\mathrm{~d}, J=7.8 \mathrm{~Hz}, 1 \mathrm{H}), 7.10-7.06(\mathrm{~m}, 2 \mathrm{H})$, $3.19(\mathrm{t}, J=12.6 \mathrm{~Hz}, 2 \mathrm{H}), 2.36(\mathrm{~s}, 3 \mathrm{H}) .{ }^{13} \mathbf{C}$ NMR $\left(150 \mathrm{MHz}, \mathrm{CDCl}_{3}\right) \delta 138.2,131.2,130.9(\mathrm{t}, J=$ $4.5 \mathrm{~Hz}), 128.7,128.4,127.4,123.0(\mathrm{t}, J=264.0 \mathrm{~Hz}), 42.2(\mathrm{t}, J=27.0 \mathrm{~Hz}), 21.3 .{ }^{19} \mathbf{F}$ NMR $(470$ $\left.\mathrm{MHz}, \mathrm{CDCl}_{3}\right) \delta-72.40(\mathrm{t}, J=12.6 \mathrm{~Hz}) . \mathbf{I R}\left(\mathrm{KBr}, \mathrm{cm}^{-1}\right) 2110$.

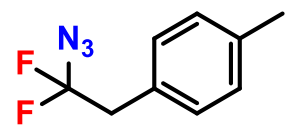

3y

1-(2-azido-2,2-difluoroethyl)-4-methylbenzene (3y) This compound was prepared according to the general procedure A. The crude mixture was purified by column chromatography with petroleum ether as the eluent to give the product $\mathbf{3 y}$ as a yellow liquid (130.1 mg, $66 \%$ yield). ${ }^{1} \mathbf{H}$ NMR $\left(600 \mathrm{MHz}, \mathrm{CDCl}_{3}\right) \delta 6.99-6.93(\mathrm{~m}, 4 \mathrm{H}), 2.99(\mathrm{t}, J=12.6 \mathrm{~Hz}, 2 \mathrm{H}), 2.15(\mathrm{~s}, 3 \mathrm{H}) .{ }^{13} \mathbf{C}$ NMR $\left(150 \mathrm{MHz}, \mathrm{CDCl}_{3}\right) \delta 137.8,130.3,129.3,128.0(\mathrm{t}, J=4.5 \mathrm{~Hz}), 123.1(\mathrm{t}, J=265.5 \mathrm{~Hz}), 41.9(\mathrm{t}, J=$ $28.5 \mathrm{~Hz}), 21.1 .{ }^{19} \mathbf{F}$ NMR $\left(470 \mathrm{MHz}, \mathrm{CDCl}_{3}\right) \delta-72.66(\mathrm{t}, J=12.6 \mathrm{~Hz}) . \mathbf{I R}\left(\mathrm{KBr}, \mathrm{cm}^{-1}\right) 2109$.<smiles>NC(F)(F)Cc1ccc(-c2ccccc2)cc1</smiles>

$3 z$

4-(2-azido-2,2-difluoroethyl)-1,1'-biphenyl (3z) This compound was prepared according to the general procedure $\mathbf{A}$. The crude mixture was purified by column chromatography with petroleum ether as the eluent to give the product $\mathbf{3 z}$ as a colorless solid (196.9 mg, 76\% yield); m.p. 110 $11{ }^{\circ} \mathrm{C} ;{ }^{1} \mathbf{H}$ NMR $\left(600 \mathrm{MHz}, \mathrm{CDCl}_{3}\right) \delta 7.60-7.56(\mathrm{~m}, 4 \mathrm{H}), 7.43(\mathrm{t}, J=7.8 \mathrm{~Hz}, 2 \mathrm{H}), 7.36-7.33$ $(\mathrm{m}, 3 \mathrm{H}), 3.27(\mathrm{t}, J=12.6 \mathrm{~Hz}, 2 \mathrm{H}) .{ }^{13} \mathbf{C}$ NMR $\left(150 \mathrm{MHz}, \mathrm{CDCl}_{3}\right) \delta 140.9,140.6,130.8,130.0(\mathrm{t}, J$ $=3.0 \mathrm{~Hz}), 128.8,127.4,127.3,127.1,123.0(\mathrm{t}, J=264.0 \mathrm{~Hz}), 41.9(\mathrm{t}, J=28.5 \mathrm{~Hz}) .{ }^{19} \mathbf{F}$ NMR $(470$ $\left.\mathrm{MHz}, \mathrm{CDCl}_{3}\right) \delta-72.38(\mathrm{t}, J=12.6 \mathrm{~Hz}) . \mathbf{I R}\left(\mathrm{KBr}, \mathrm{cm}^{-1}\right) 2107$.<smiles>NC(F)(F)Cc1ccc(F)cc1</smiles>

1-(2-azido-2,2-difluoroethyl)-4-fluorobenzene (3aa) This compound was prepared according to the general procedure $\mathbf{A}$. The crude mixture was purified by column chromatography with petroleum ether as the eluent to give the product 3aa as a yellow liquid (142.8 mg, $71 \%$ yield). ${ }^{1} \mathbf{H}$ NMR $\left(600 \mathrm{MHz}, \mathrm{CDCl}_{3}\right) \delta 7.27-7.23(\mathrm{~m}, 2 \mathrm{H}), 7.03(\mathrm{t}, J=8.5 \mathrm{~Hz}, 2 \mathrm{H}), 3.20(\mathrm{t}, J=12.6 \mathrm{~Hz}, 2 \mathrm{H})$. ${ }^{13} \mathbf{C}$ NMR $\left(150 \mathrm{MHz}, \mathrm{CDCl}_{3}\right) \delta 162.6(\mathrm{~d}, J=244.5 \mathrm{~Hz}), 132.1(\mathrm{~d}, J=7.5 \mathrm{~Hz}), 126.8(\mathrm{q}, J=3.0$ $\mathrm{Hz}), 122.8(\mathrm{t}, J=268.0 \mathrm{~Hz}), 115.5(\mathrm{~d}, J=21.0 \mathrm{~Hz}), 41.4(\mathrm{t}, J=27.0 \mathrm{~Hz}) .{ }^{19} \mathbf{F}$ NMR $(470 \mathrm{MHz}$, $\left.\mathrm{CDCl}_{3}\right) \delta-72.89(\mathrm{t}, J=12.6 \mathrm{~Hz}),-114.24--114.38(\mathrm{~m}) . \mathbf{I R}\left(\mathrm{KBr}, \mathrm{cm}^{-1}\right) 2109$. 
<smiles>NC(F)(F)Cc1ccc(Cl)cc1</smiles>

$3 a b$

1-(2-azido-2,2-difluoroethyl)-4-chlorobenzene (3ab) This compound was prepared according to the general procedure A. The crude mixture was purified by column chromatography with petroleum ether as the eluent to give the product $\mathbf{3 a b}$ as a yellow liquid (154.1 mg, $71 \%$ yield). ${ }^{1} \mathbf{H}$ NMR $\left(600 \mathrm{MHz}, \mathrm{CDCl}_{3}\right) \delta 7.32(\mathrm{~d}, J=8.4 \mathrm{~Hz}, 2 \mathrm{H}), 7.21(\mathrm{~d}, J=8.4 \mathrm{~Hz}, 2 \mathrm{H}), 3.20(\mathrm{t}, J=12.6 \mathrm{~Hz}$, 2H). ${ }^{13} \mathbf{C}$ NMR $\left(150 \mathrm{MHz}, \mathrm{CDCl}_{3}\right) \delta 134.1,131.7,129.5$ (t, $\left.J=3.0 \mathrm{~Hz}\right), 128.7,122.7$ (t, $J=265.5$ $\mathrm{Hz}), 41.6(\mathrm{t}, J=27.0 \mathrm{~Hz}) .{ }^{19} \mathbf{F}$ NMR $\left(470 \mathrm{MHz}, \mathrm{CDCl}_{3}\right) \delta-72.61(\mathrm{t}, J=12.6 \mathrm{~Hz}) . \mathbf{I R}\left(\mathrm{KBr}, \mathrm{cm}^{-1}\right)$ 2110 .<smiles>NC(F)(F)Cc1ccc(Br)cc1</smiles>

3ac

1-(2-azido-2,2-difluoroethyl)-4-bromobenzene (3ac) This compound was prepared according to the general procedure A. The crude mixture was purified by column chromatography with petroleum ether as the eluent to give the product 3ac as a yellow liquid (190.5 mg, $73 \%$ yield). ${ }^{1} \mathbf{H}$ NMR $\left(600 \mathrm{MHz}, \mathrm{CDCl}_{3}\right) \delta 7.47$ (d, $\left.J=8.4 \mathrm{~Hz}, 2 \mathrm{H}\right), 7.15(\mathrm{~d}, J=8.4 \mathrm{~Hz}, 2 \mathrm{H}), 3.18$ (t, $J=12.6 \mathrm{~Hz}$, 2H). ${ }^{13} \mathbf{C ~ N M R}\left(150 \mathrm{MHz}, \mathrm{CDCl}_{3}\right) \delta 132.1,131.7,130.0(\mathrm{t}, J=3.0 \mathrm{~Hz}), 122.6(\mathrm{t}, J=265.5 \mathrm{~Hz})$, 122.2, $41.6(\mathrm{t}, J=27.0 \mathrm{~Hz}) .{ }^{19} \mathbf{F}$ NMR $\left(470 \mathrm{MHz}, \mathrm{CDCl}_{3}\right) \delta-72.56(\mathrm{t}, J=12.6 \mathrm{~Hz}) . \mathbf{I R}\left(\mathrm{KBr}, \mathrm{cm}^{-1}\right)$ 2107.

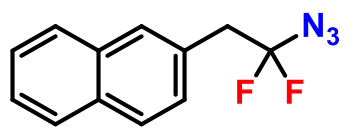

3ad

2-(2-azido-2,2-difluoroethyl)naphthalene (3ad) This compound was prepared according to the general procedure $\mathbf{A}$. The crude mixture was purified by column chromatography with petroleum ether as the eluent to give the product $\mathbf{3 a d}$ as a colorless liquid (144.5 mg, 62\% yield). ${ }^{1} \mathbf{H}$ NMR $\left(600 \mathrm{MHz}, \mathrm{CDCl}_{3}\right) \delta 7.84-7.81(\mathrm{~m}, 3 \mathrm{H}), 7.74(\mathrm{~s}, 1 \mathrm{H}), 7.52-7.46(\mathrm{~m}, 2 \mathrm{H}), 7.39(\mathrm{~d}, J=8.4 \mathrm{~Hz}$, $1 \mathrm{H}), 3.39(\mathrm{t}, J=12.0 \mathrm{~Hz}, 2 \mathrm{H}) .{ }^{13} \mathbf{C}$ NMR $\left(150 \mathrm{MHz}, \mathrm{CDCl}_{3}\right) \delta 133.2,132.8,129.7,128.5(\mathrm{t}, J=$ $3.0 \mathrm{~Hz}), 128.2,128.0,127.8,127.7,126.3,126.2,123.1(\mathrm{t}, J=265.5 \mathrm{~Hz}), 42.3(\mathrm{t}, J=27.0 \mathrm{~Hz}) .{ }^{19} \mathbf{F}$ NMR $\left(470 \mathrm{MHz}, \mathrm{CDCl}_{3}\right) \delta-72.13(\mathrm{t}, J=12.0 \mathrm{~Hz}) . \mathbf{I R}\left(\mathrm{KBr}, \mathrm{cm}^{-1}\right) 2109$.

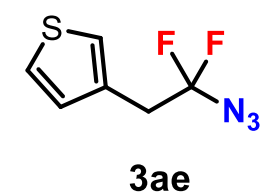

3-(2-azido-2,2-difluoroethyl)thiophene (3ae) This compound was prepared according to the general procedure $\mathbf{A}$. The crude mixture was purified by column chromatography with petroleum ether as the eluent to give the product 3ae as a colorless liquid (107.8 mg, 57\% yield). ${ }^{\mathbf{1}} \mathbf{H}$ NMR $\left(600 \mathrm{MHz}, \mathrm{CDCl}_{3}\right) \delta 7.31(\mathrm{dd}, J=4.8,3.0 \mathrm{~Hz}, 1 \mathrm{H}), 7.20(\mathrm{~d}, J=3.0 \mathrm{~Hz}, 1 \mathrm{H}), 7.04(\mathrm{~d}, J=4.8 \mathrm{~Hz}$, $1 \mathrm{H}), 3.29(\mathrm{t}, J=12.6 \mathrm{~Hz}, 2 \mathrm{H}) .{ }^{13} \mathbf{C}$ NMR $\left(150 \mathrm{MHz}, \mathrm{CDCl}_{3}\right) \delta 130.9(\mathrm{t}, J=3.0 \mathrm{~Hz}), 128.9,125.9$, 125.0, $122.6(\mathrm{t}, J=265.5 \mathrm{~Hz}), 36.9(\mathrm{t}, J=28.5 \mathrm{~Hz}) .{ }^{19} \mathbf{F}$ NMR $\left(470 \mathrm{MHz}, \mathrm{CDCl}_{3}\right) \delta-72.76(\mathrm{t}, J=$ $12.6 \mathrm{~Hz}) . \mathbf{I R}\left(\mathrm{KBr}, \mathrm{cm}^{-1}\right) 2103$. 


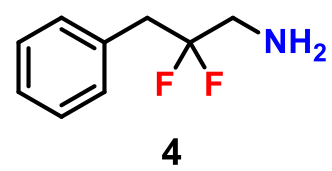

2,2-difluoro-3-phenylpropan-1-amine (4) This compound was prepared according to the general procedure $\mathbf{B}$. The crude mixture was purified by column chromatography with petroleum ether as the eluent to give the product $\mathbf{4}$ as a colorless liquid (166.0 mg, 97\% yield). ${ }^{1} \mathbf{H} \mathbf{~ N M R}$ (600 MHz, $\left.\mathrm{CDCl}_{3}\right) \delta 7.36-7.25(\mathrm{~m}, 5 \mathrm{H}), 3.19(\mathrm{t}, J=16.2 \mathrm{~Hz}, 2 \mathrm{H}), 2.89(\mathrm{t}, J=15.0 \mathrm{~Hz}, 2 \mathrm{H}), 1.30(\mathrm{~s}, 2 \mathrm{H}) .{ }^{13} \mathrm{C}$ $\operatorname{NMR}\left(150 \mathrm{MHz}, \mathrm{CDCl}_{3}\right) \delta 133.0(\mathrm{t}, J=4.5 \mathrm{~Hz}), 130.2,128.5,127.3,123.0(\mathrm{t}, J=240.0 \mathrm{~Hz}), 46.1(\mathrm{t}, J$ $=27.0 \mathrm{~Hz}), 40.8(\mathrm{t}, J=25.5 \mathrm{~Hz}) .{ }^{19} \mathbf{F}$ NMR $\left(470 \mathrm{MHz}, \mathrm{CDCl}_{3}\right) \delta-105.49(\mathrm{p}, J=15.5 \mathrm{~Hz}) . \mathbf{H R M S}$ (ESI) $\mathrm{m} / \mathrm{z}$ calcd. for $\mathrm{C}_{9} \mathrm{H}_{11} \mathrm{~F}_{2} \mathrm{NNa}[\mathrm{M}+\mathrm{Na}]^{+}$194.0757, found 194.0754.

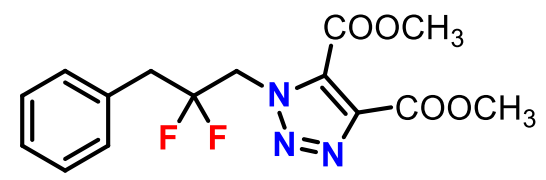

5

dimethyl 1-(2,2-difluoro-3-phenylpropyl)-1H-1,2,3-triazole-4,5-dicarboxylate (5) This compound was prepared according to the general procedure $\mathbf{C}$. The crude mixture was purified by column chromatography with ethyl acetate/petroleum ether $=1: 5$ as the eluent to give the product 5 as a colorless solid (318.7 mg, 94\% yield); m.p. $96-97{ }^{\circ} \mathrm{C} ;{ }^{1} \mathbf{H}$ NMR $\left(600 \mathrm{MHz}, \mathrm{CDCl}_{3}\right) \delta 7.40$ - $7.34(\mathrm{~m}, 3 \mathrm{H}), 7.33$ (d, $J=7.2 \mathrm{~Hz}, 2 \mathrm{H}), 4.99$ (t, $J=13.2 \mathrm{~Hz}, 2 \mathrm{H}), 3.99$ (s, 3H), 3.95 (s, 3H), 3.22 $(\mathrm{t}, J=16.2 \mathrm{~Hz}, 2 \mathrm{H}) .{ }^{13} \mathbf{C} \mathbf{N M R}\left(150 \mathrm{MHz}, \mathrm{CDCl}_{3}\right) \delta 160.1,158.8,139.8,131.6,130.9$ (t, $J=4.5$ $\mathrm{Hz}), 130.5,128.8,128.1,119.7(\mathrm{t}, J=246.0 \mathrm{~Hz}), 53.5,52.8,51.9(\mathrm{t}, J=28.5 \mathrm{~Hz}), 41.0$ (t, $J=24.0$ Hz). ${ }^{19} \mathbf{F}$ NMR (470 MHz, $\left.\mathrm{CDCl}_{3}\right) \delta-100.88--101.01$ (m). HRMS (ESI) $\mathrm{m} / \mathrm{z}$ calcd. for $\mathrm{C}_{15} \mathrm{H}_{15} \mathrm{~F}_{2} \mathrm{~N}_{3} \mathrm{O}_{4} \mathrm{Na}[\mathrm{M}+\mathrm{Na}]^{+}$362.0928, found 362.0926.<smiles>[2H]C([2H])=C(N)c1ccc(C)cc1</smiles>

\section{$\left[D_{2}\right]-6$}

$\left[\mathbf{D}_{2}\right]$-6: This compound was prepared according to the general procedure $\mathbf{D}$. The crude mixture was purified by column chromatography with petroleum ether as the eluent to give the product [D $\left.\mathbf{D}_{2}\right]-6$ as a yellow liquid (130.5 mg, $81 \%$ yield). ${ }^{1} \mathbf{H} \mathbf{~ N M R ~}\left(600 \mathrm{MHz}, \mathrm{CDCl}_{3}\right) \delta 7.44(\mathrm{~d}, J=8.4$ $\mathrm{Hz}, 2 \mathrm{H}), 7.15(\mathrm{~d}, J=8.4 \mathrm{~Hz}, 2 \mathrm{H}), 5.37-5.35(\mathrm{~m}, 0.49 \mathrm{H}), 4.90-4.88(\mathrm{~m}, 0.27 \mathrm{H}), 2.35(\mathrm{~s}, 3 \mathrm{H})$.<smiles>[2H]C([2H])(c1ccc(C)cc1)C([2H])(N)C(F)(F)F</smiles>

\section{$\left[\mathrm{D}_{2}\right]-3 \mathrm{y}$}

$\left[\mathbf{D}_{2}\right]-3 \mathbf{y}$ : This compound was prepared according to the general procedure $\mathbf{E}$. The crude mixture was purified by column chromatography with petroleum ether as the eluent to give the product $\left[\mathbf{D}_{2}\right]-3 \mathbf{y}$ as a yellow liquid $\left(125.4 \mathrm{mg}, 63 \%\right.$ yield). ${ }^{1} \mathbf{H} \mathbf{~ N M R}\left(600 \mathrm{MHz}, \mathrm{CDCl}_{3}\right) \delta 7.11-7.07(\mathrm{~m}$, 4H), $3.15-3.09$ (m, 0.83H), 2.28 (s, 3H). ${ }^{13} \mathbf{C}$ NMR $\left(150 \mathrm{MHz}, \mathrm{CDCl}_{3}\right) \delta$ 137.7, 130.3, 129.2, 
$128.2-127.5(\mathrm{~m}), 123.0(\mathrm{t}, J=266.5 \mathrm{~Hz}), 41.8-41.3(\mathrm{~m}), 21.1 .{ }^{19} \mathbf{F} \mathbf{N M R}\left(470 \mathrm{MHz}, \mathrm{CDCl}_{3}\right) \delta$ $-72.64--72.89(\mathrm{~m})$.

\section{Elemental Analysis of Compound 3z}

\begin{tabular}{|c|c|c|c|}
\hline \multicolumn{4}{|c|}{$\mathbf{C}_{14} \mathbf{H}_{11} \mathbf{F}_{2} \mathbf{N}_{3}$} \\
\hline $\mathbf{N}(\%)$ & $\mathrm{C}(\%)$ & $\mathbf{H}(\%)$ & $\mathrm{F}(\%)$ \\
\hline 16.158 & 61.947 & 4.056 & 17.839 \\
\hline
\end{tabular}




\section{Copies of NMR spectra}
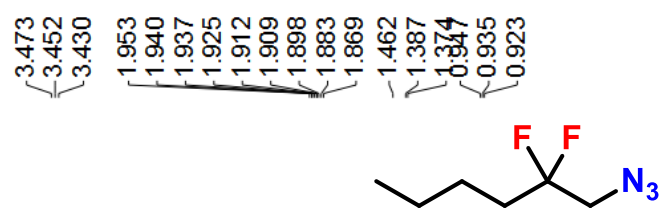

$2 a$
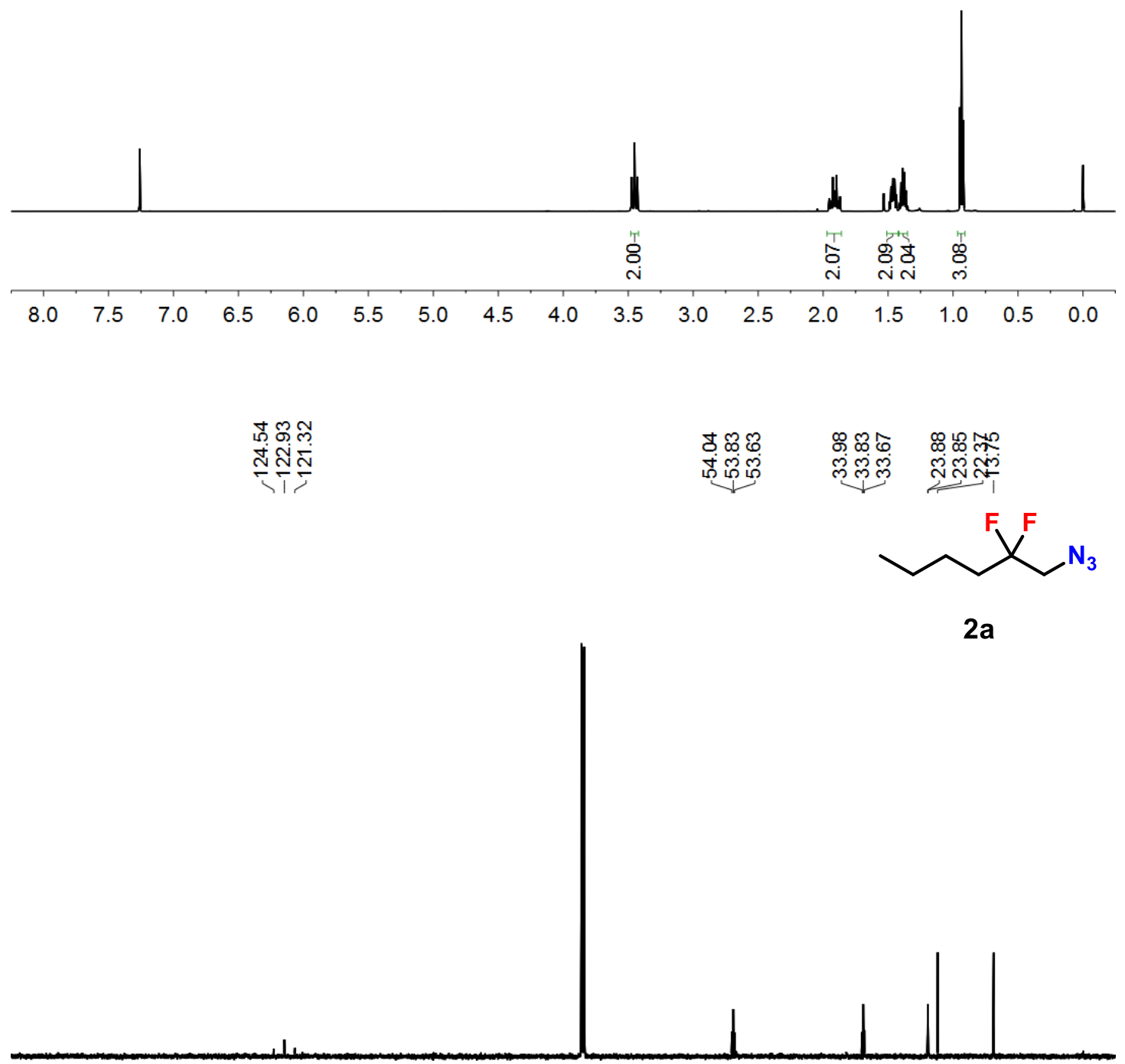

$\begin{array}{lllllllllllllllll}160 & 150 & 140 & 130 & 120 & 110 & 100 & 90 & 80 & 70 & 60 & 50 & 40 & 30 & 20 & 10 & 0\end{array}$ 


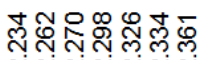

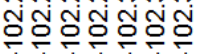

i们

$\sim^{\mathrm{F}} \mathrm{N}_{3}^{\mathrm{F}}$

$2 a$

MVM

\begin{tabular}{lllll}
\hline-101.85 & -102.10 & -102.35 & -102.60 & -102.85
\end{tabular}

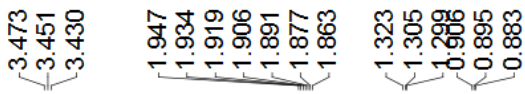

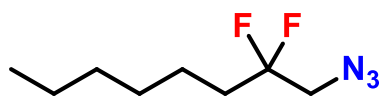

2b

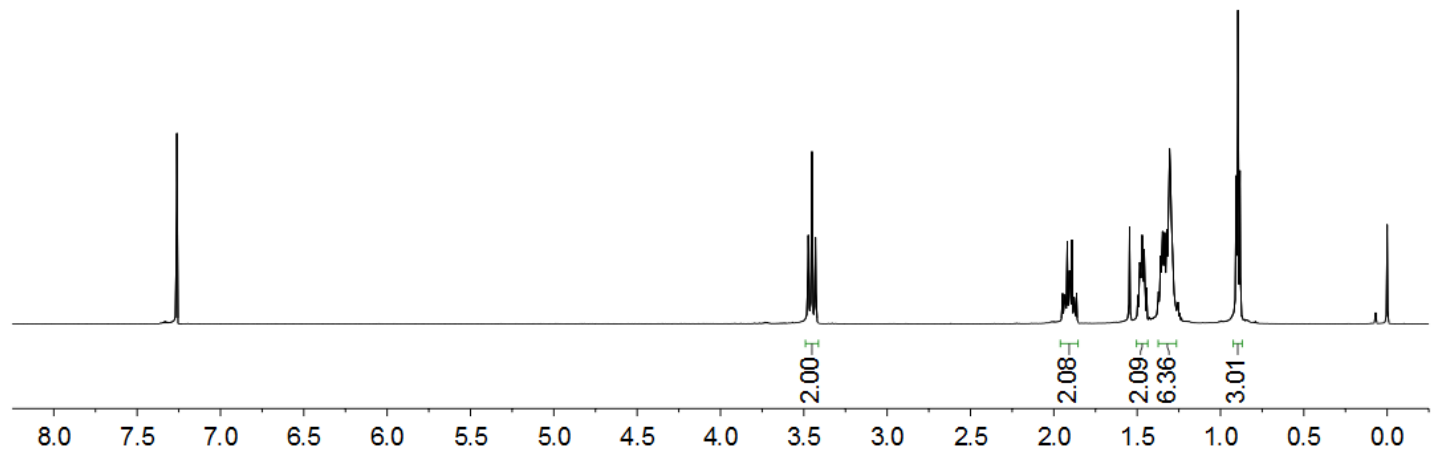




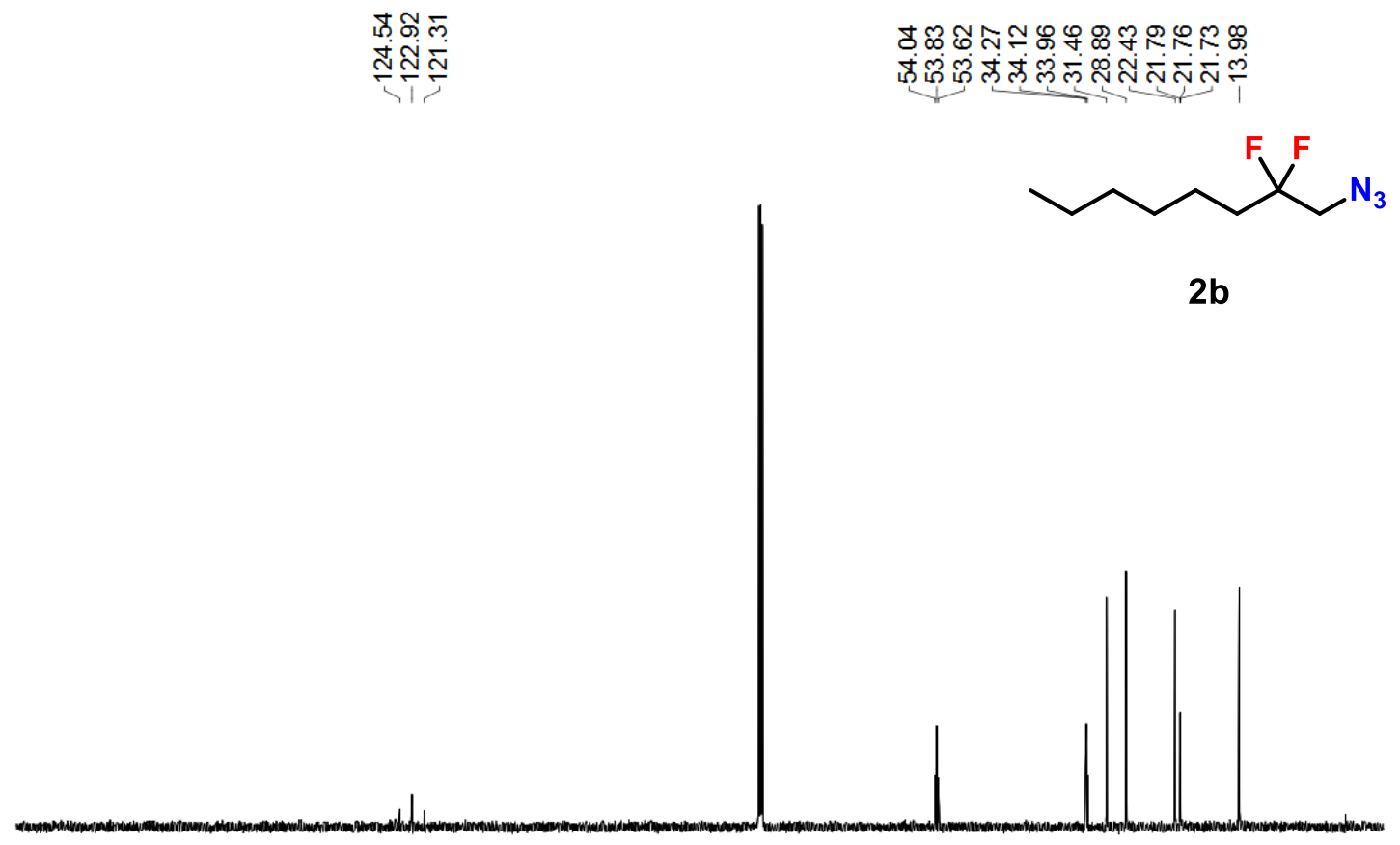

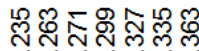

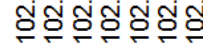

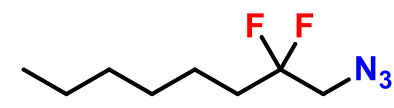

$2 b$

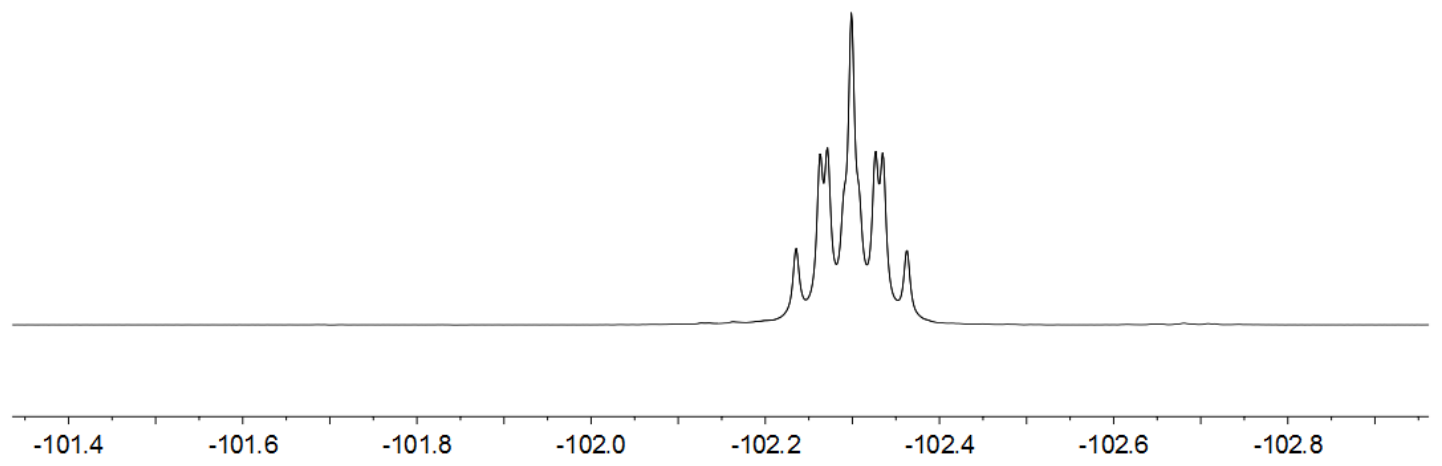




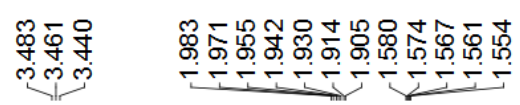

$\underbrace{N_{F}}_{F} \overbrace{N_{3}}^{F}$

2c

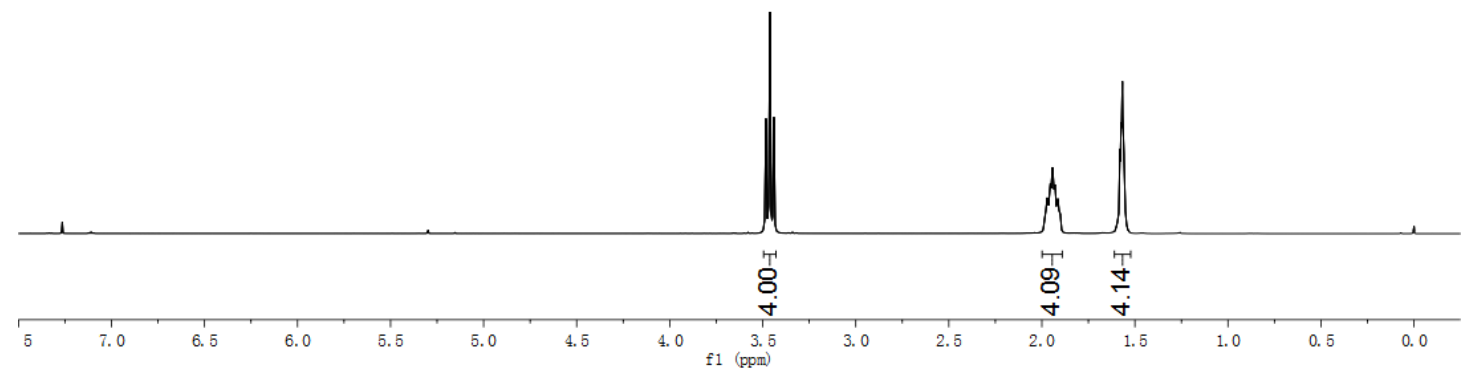

స⿻ำ

ปู่ สิ่

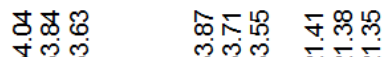

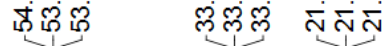

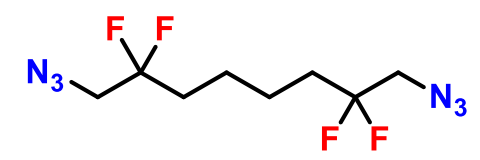

2c

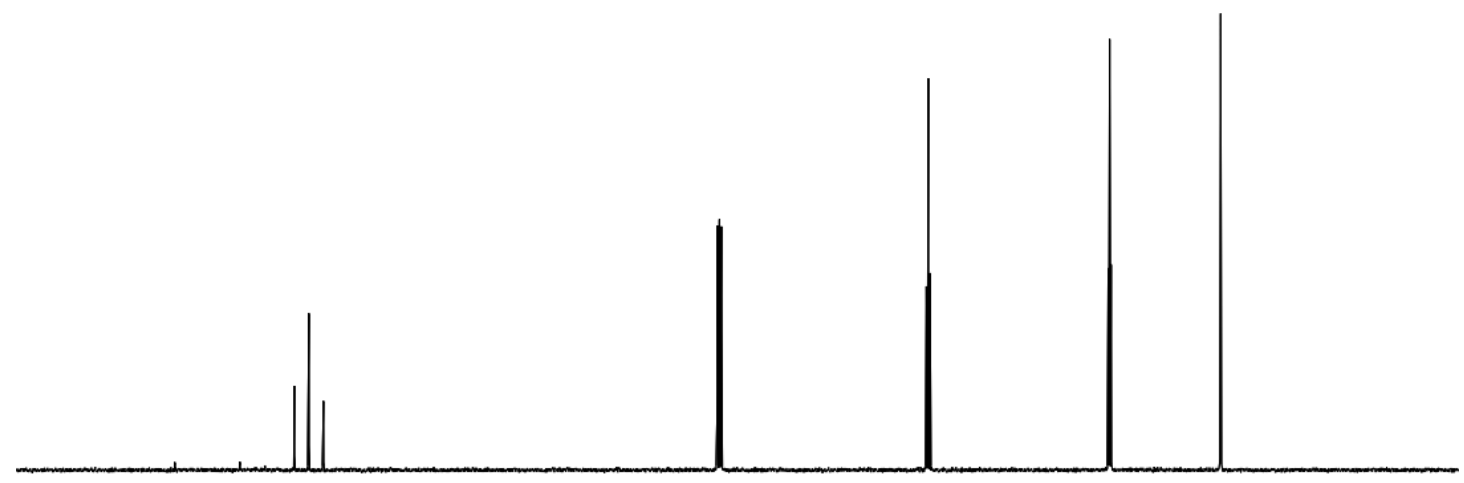

150

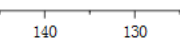

120

$110 \quad 100$

90

${ }_{\mathrm{f} 1(\mathrm{ppm})}{ }^{70}$ 

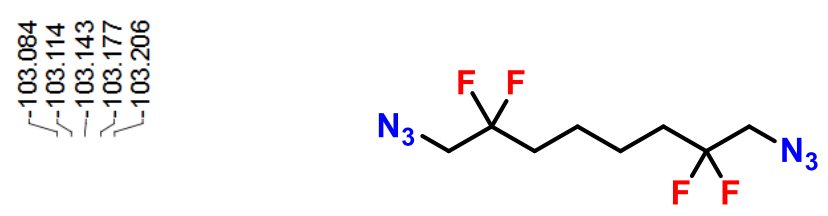

2c

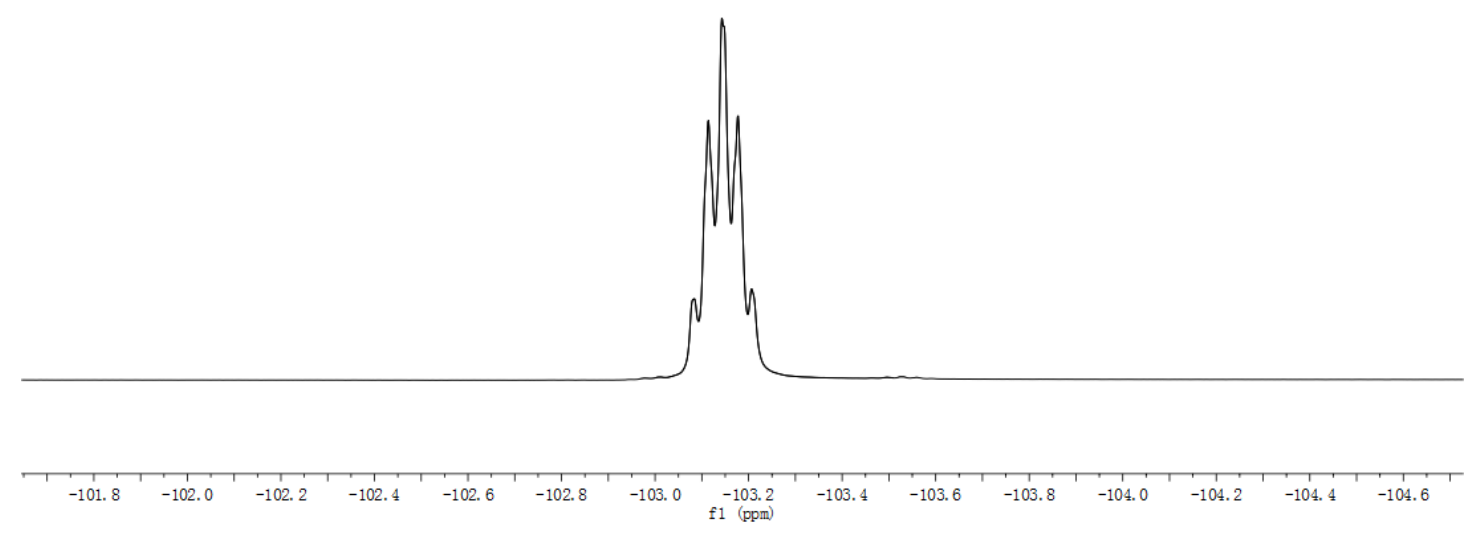

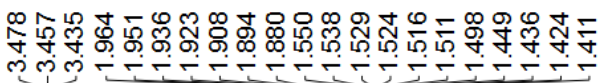

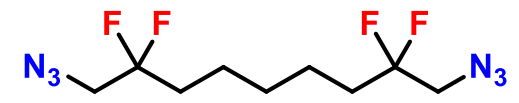

2d

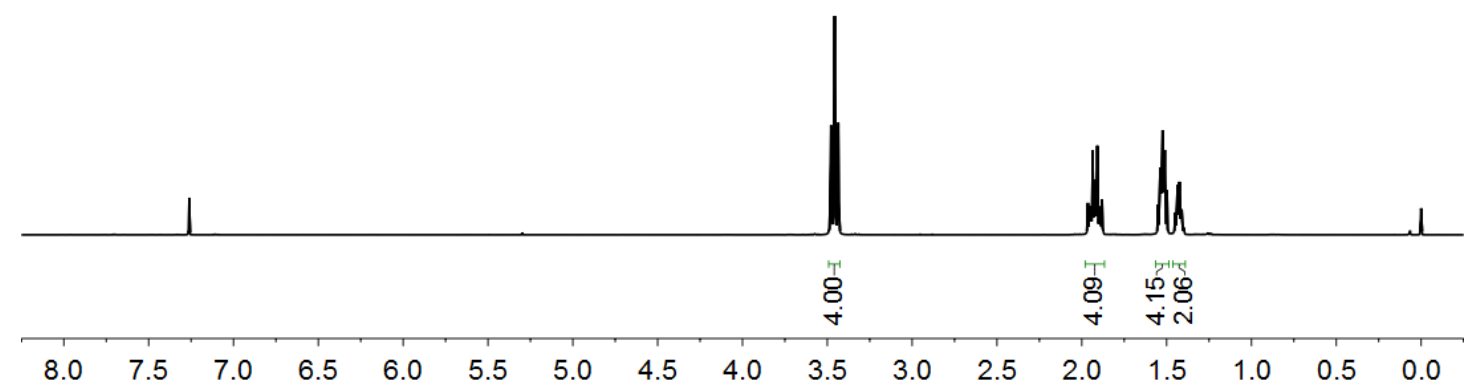


뜌용

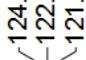

क人ष

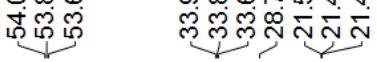

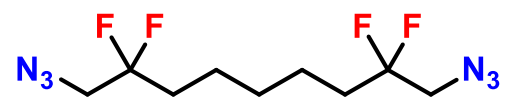

2d

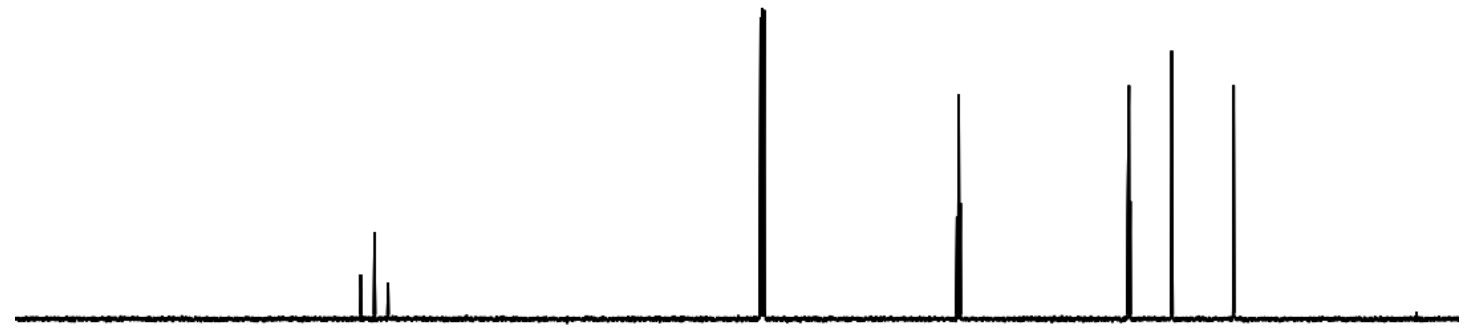

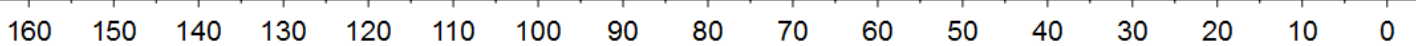

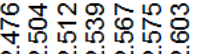

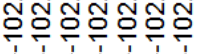<smiles>NCC(F)(F)CCCCCC(F)(F)CN</smiles>

2d

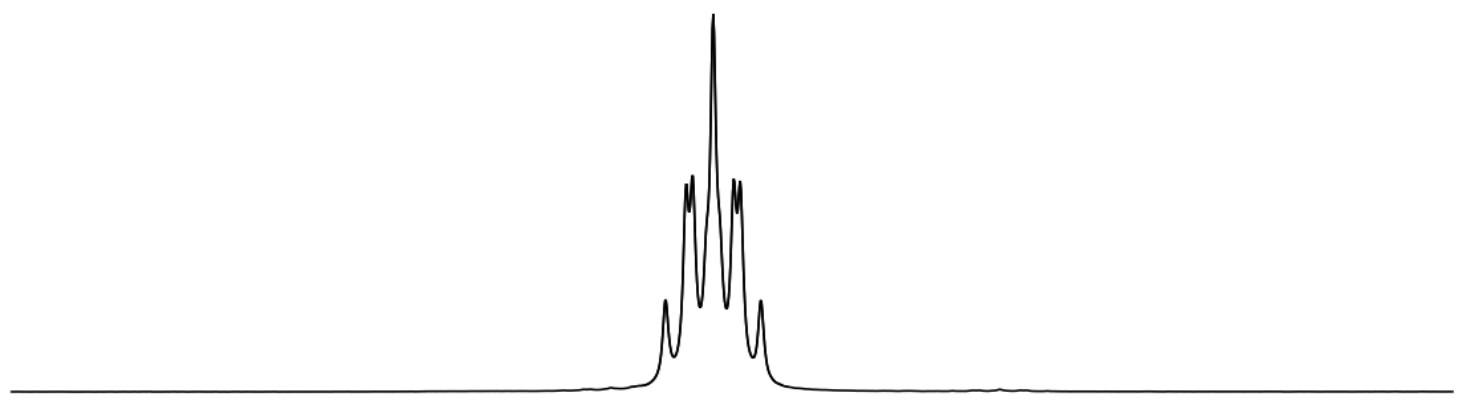

$-101.8$ $-102.0$ $-102.2$ $-1024$ $-102.6$ $-102.8$ $-103.0$ $-103.2-103.4$ 


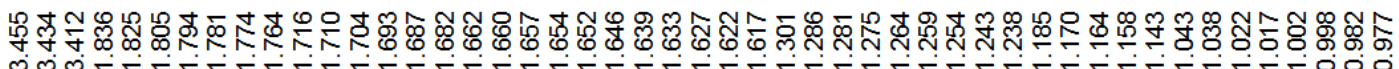<smiles>NCC(F)(F)CC1CCCCC1</smiles>

$2 e$

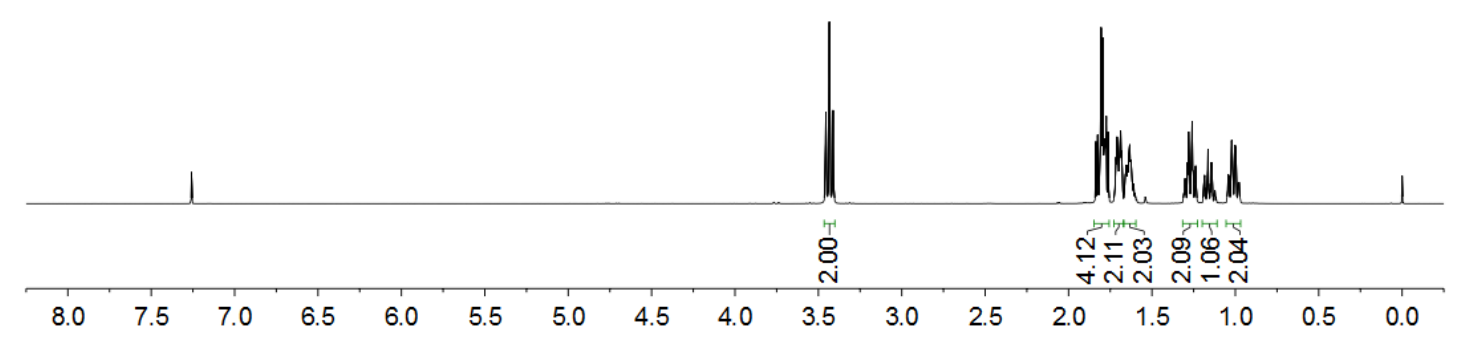

용요용

ป્d

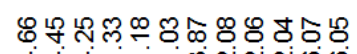

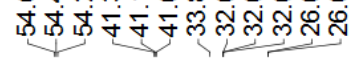

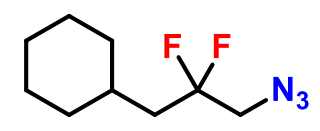

$2 e$

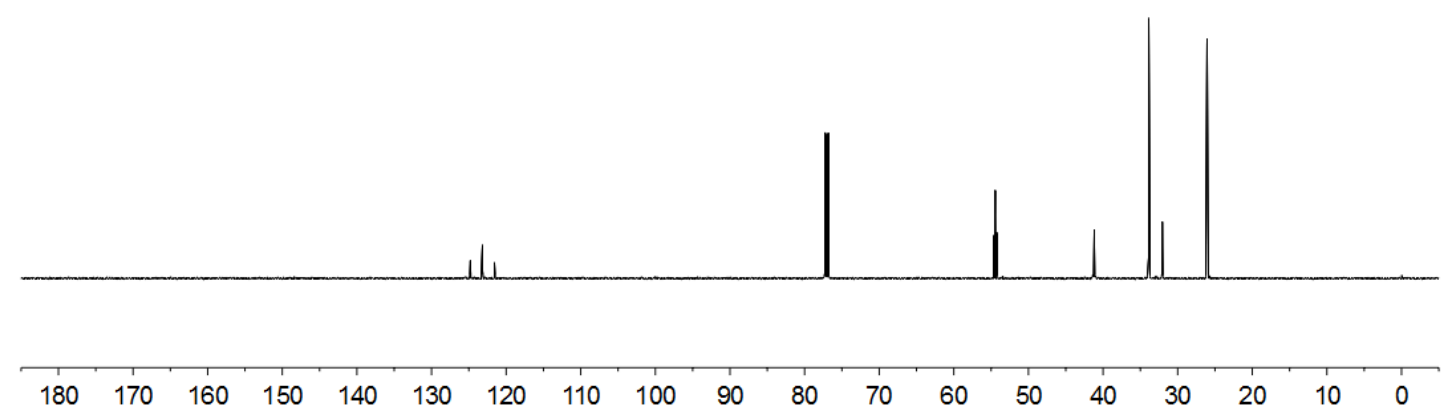




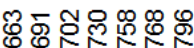

क्ष

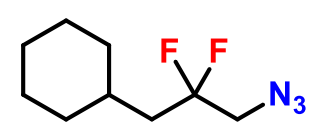

$2 e$

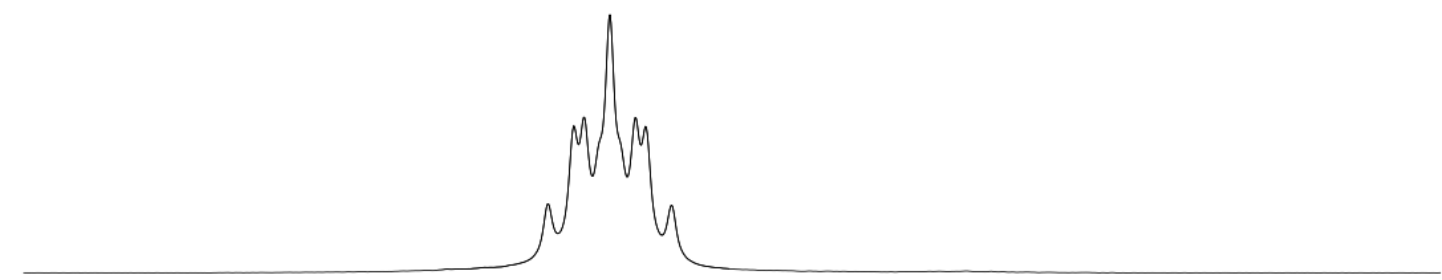

$-99.15$

$-99.35$

$-99.55$

$-99.75$

$-99.95$

$-100.15$

$-100.40$

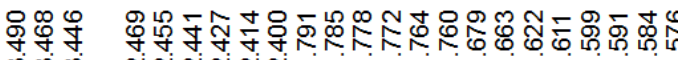

m<smiles>[N]CC(F)(F)C1CCCC1</smiles>

$2 f$

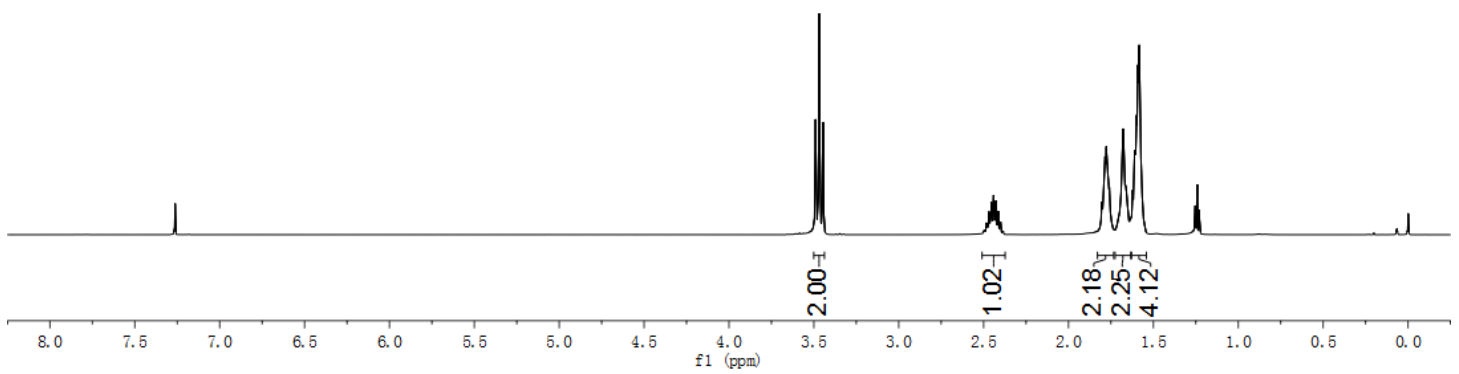




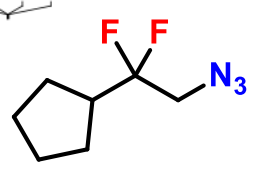

$2 f$
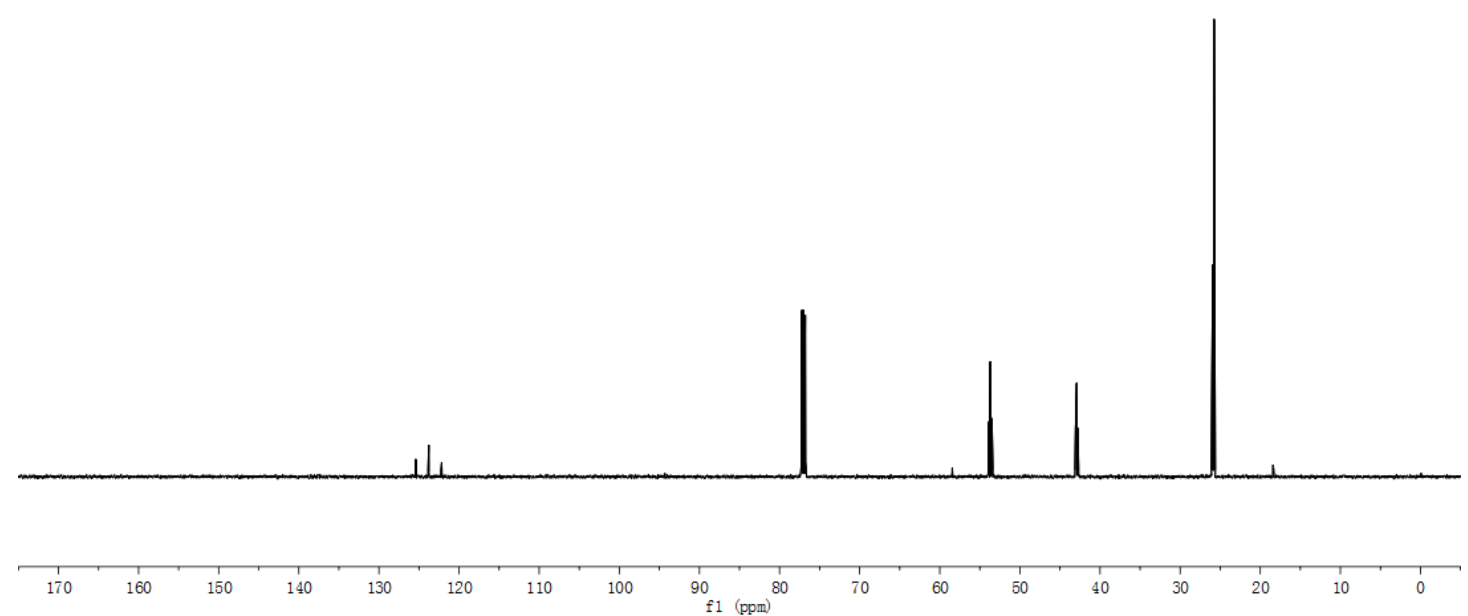

\section{훙용}

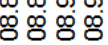

(T)

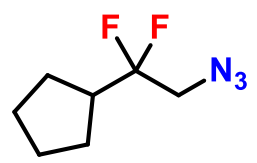

$2 f$

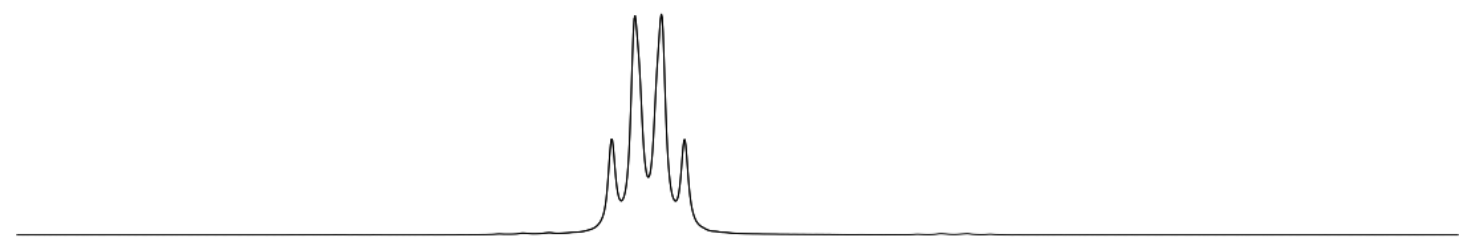

\begin{tabular}{|lllllllllllllllllll}
\hline 8.1 & -108.2 & -108.3 & -108.4 & -108.5 & -108.6 & -108.7 & -108.8 & -108.9 & -109.0 & -109.1 & -109.2 & -109.3 & -109.4 & -109.5 & -109.6 & -109.7 & -109.8 & 1
\end{tabular} 


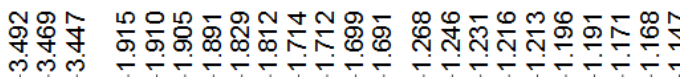

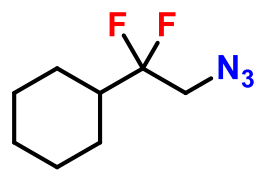

$2 g$

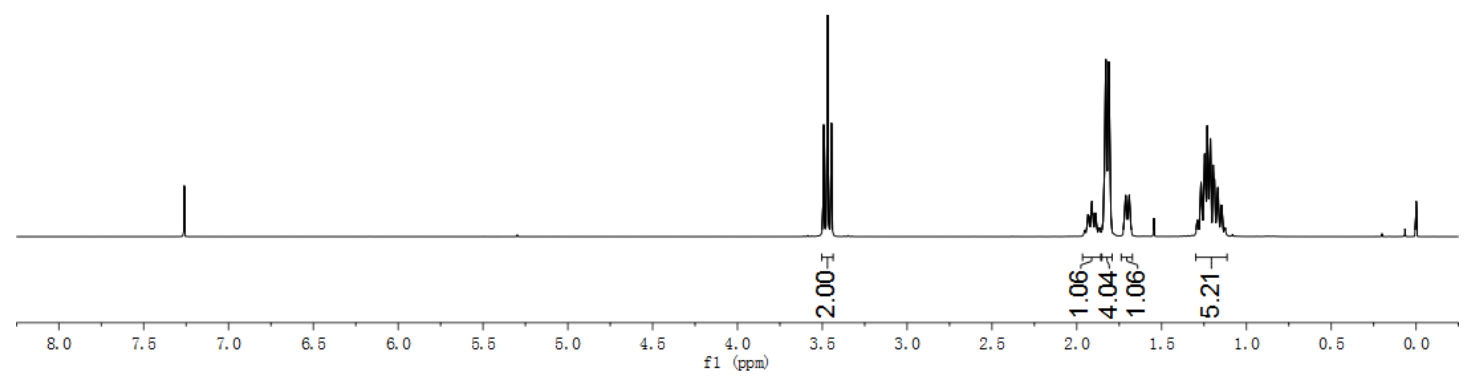

ஸุढ

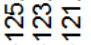

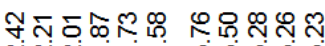

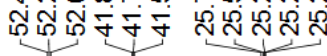

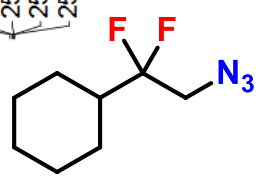

2g

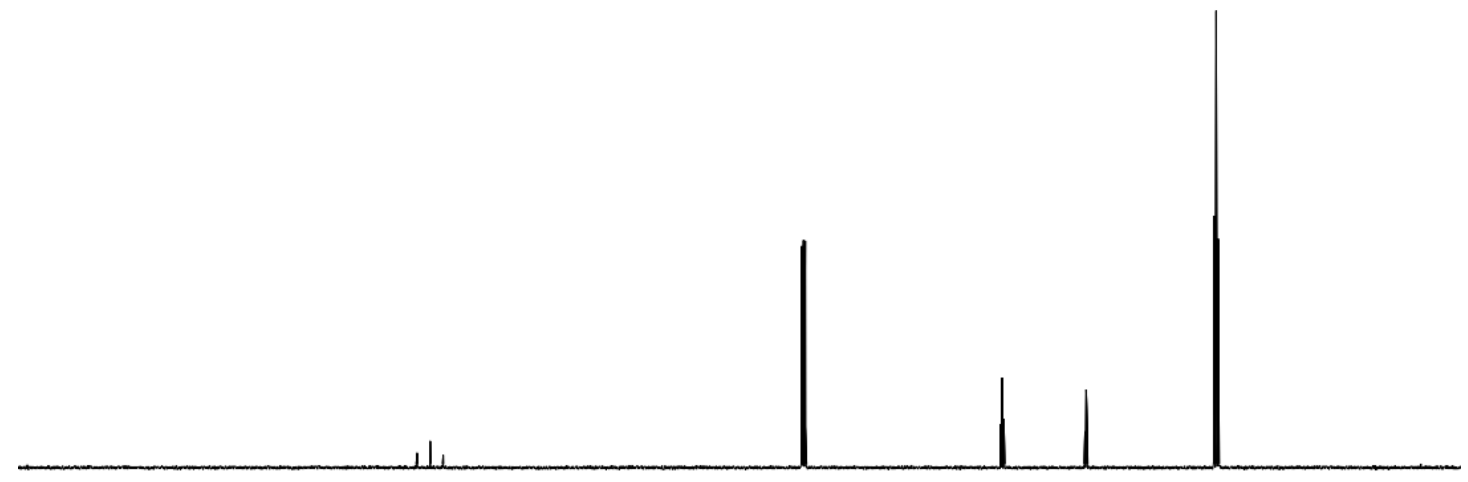

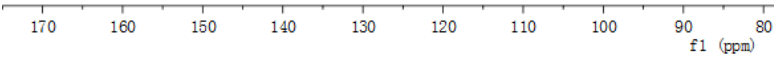




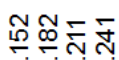

웅웅음

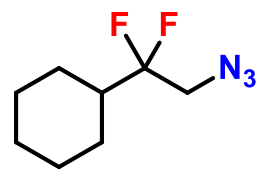

$2 \mathrm{~g}$

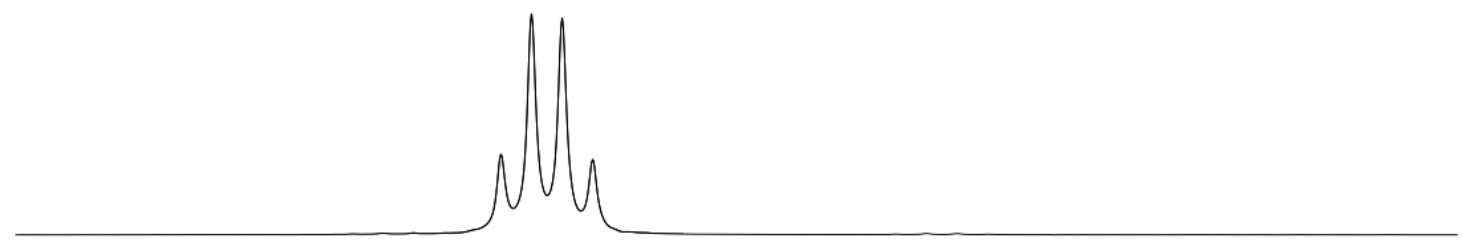

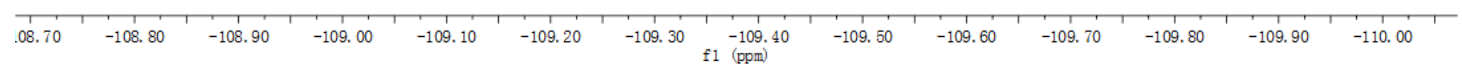

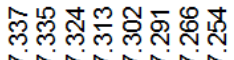

율율 뜸 중

ल्लिंलुल

$\bigoplus \mathrm{F}_{\mathrm{F}} \mathrm{N}_{3}$

2h

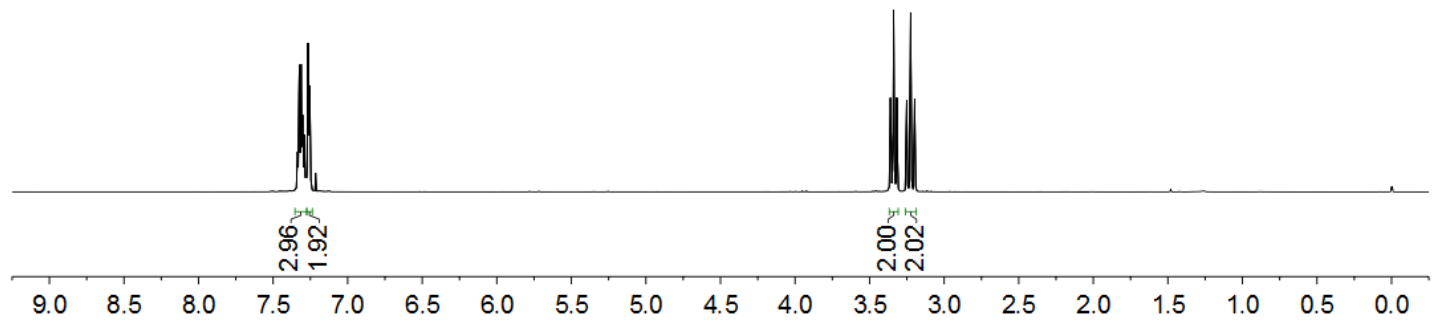


<smiles>[N]CC(F)(F)Cc1ccccc1</smiles>

$2 \mathrm{~h}$
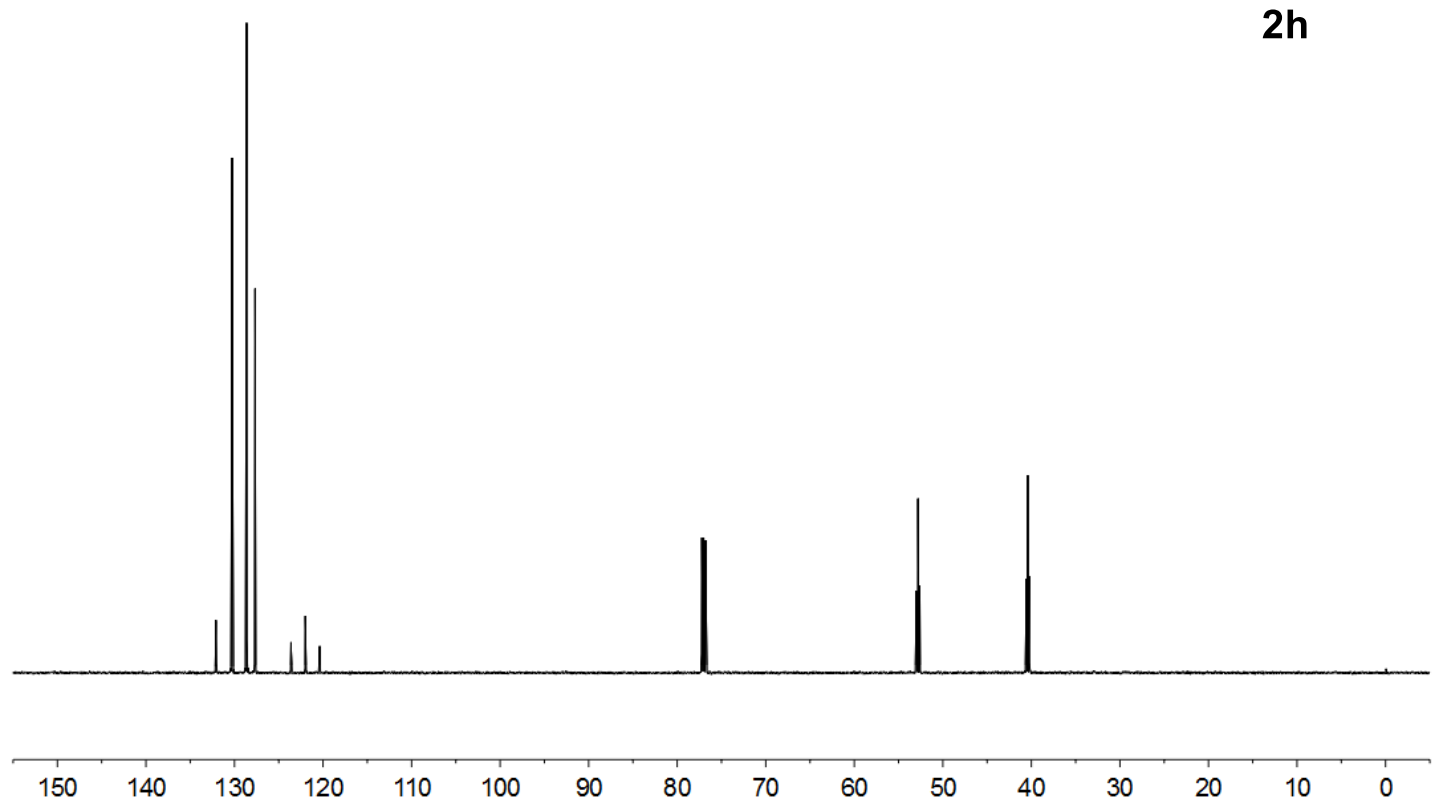

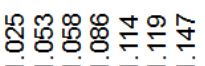 \\ 훈훈휘훙후}

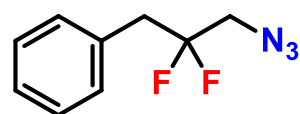

$2 \mathrm{~h}$

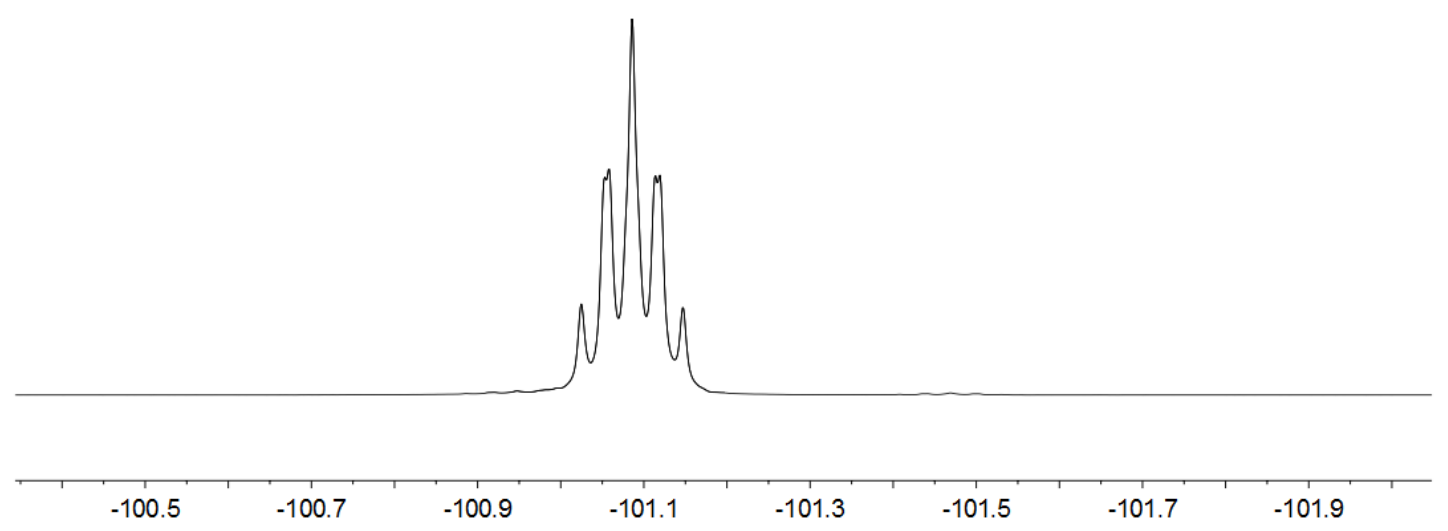




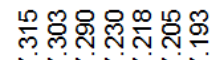

NNNNN

$\stackrel{2}{2}$

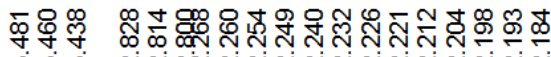

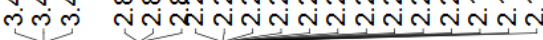<smiles>NCC(F)(F)CCc1ccccc1</smiles>

$2 \mathbf{i}$

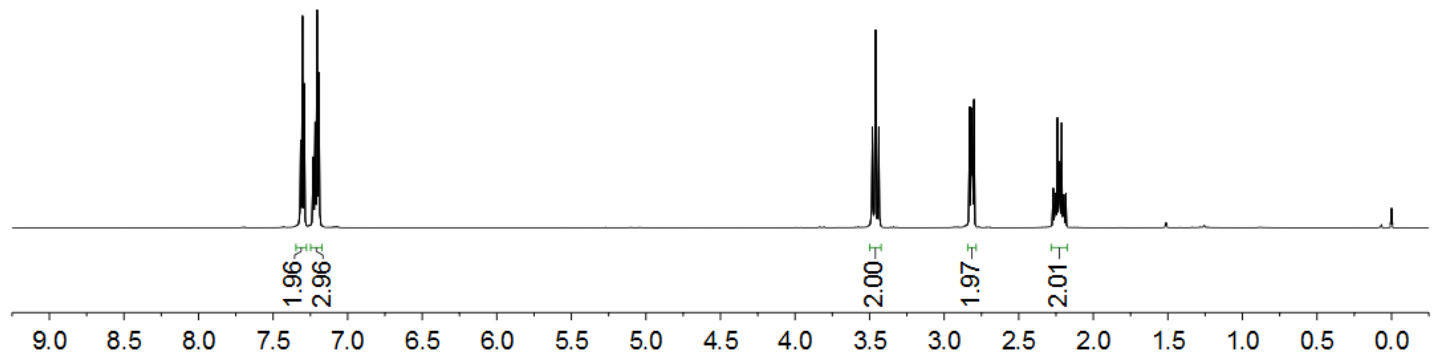

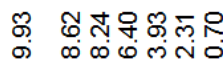

ตับ ํํำ ํํำ

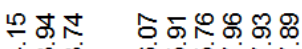

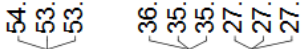<smiles>NCC(F)(F)CCc1ccccc1</smiles>

2i

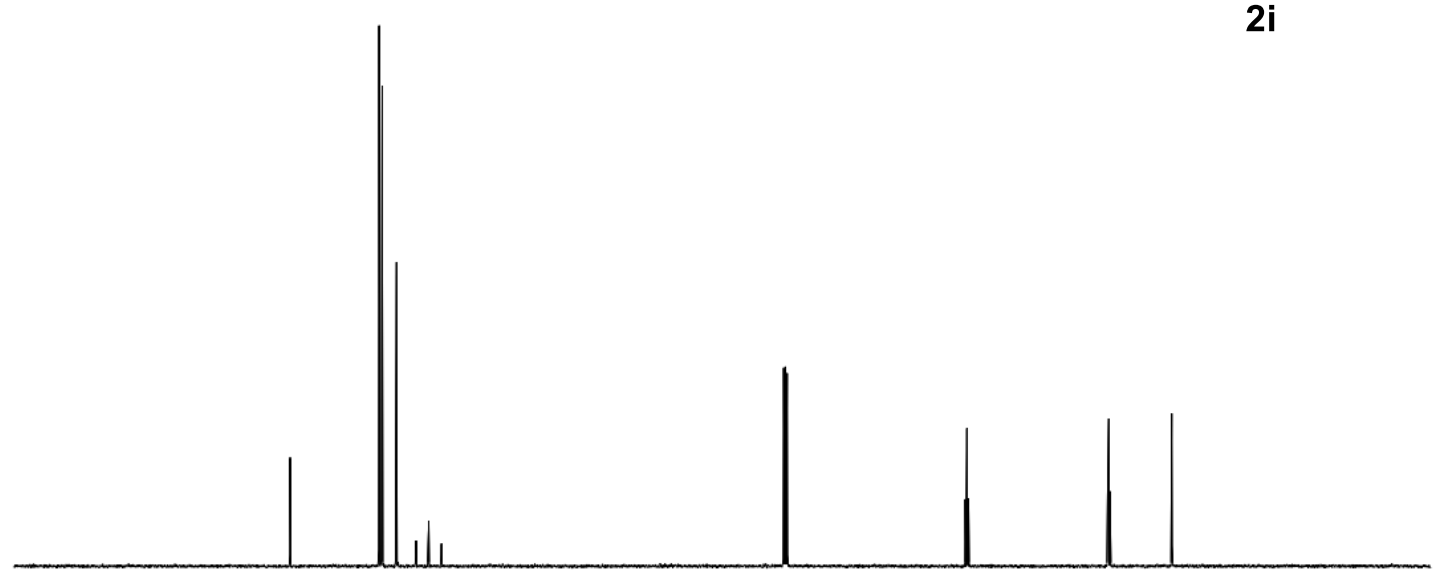

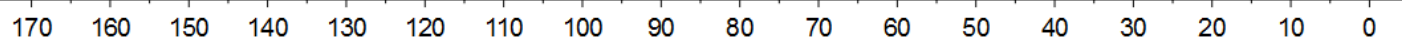




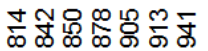

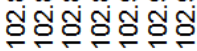

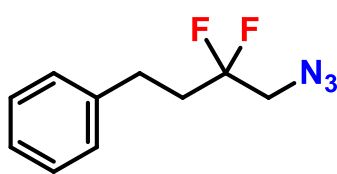

$2 \mathbf{i}$

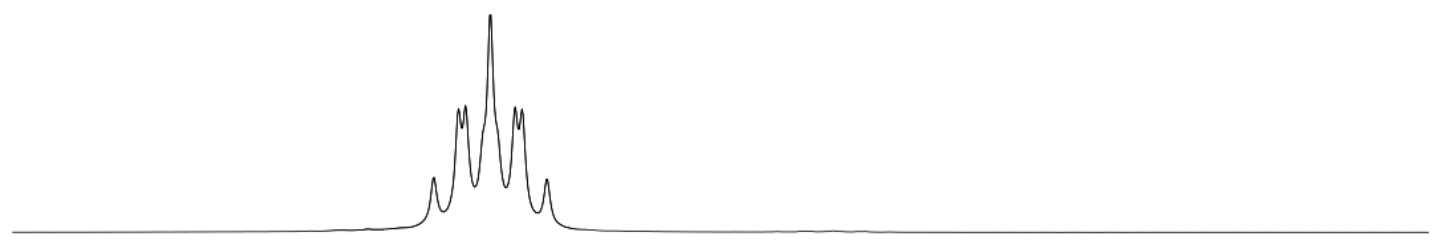

\begin{tabular}{ll|l|l|l|l|l|l|}
\hline-102.4 & -102.6 & -102.8 & -103.0 & -103.2 & -103.4 & -103.6 & -103.8 \\
\hline
\end{tabular}

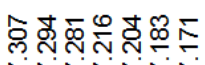

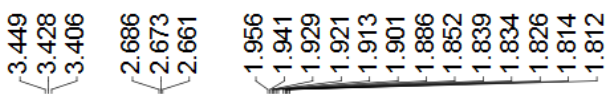

NNNNN

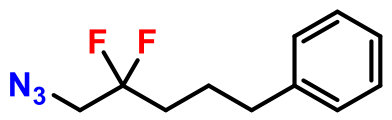

2j

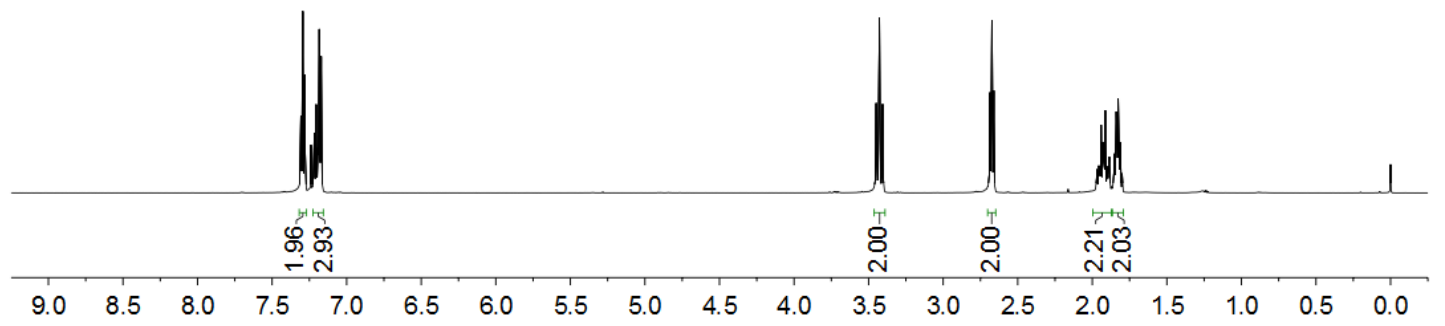




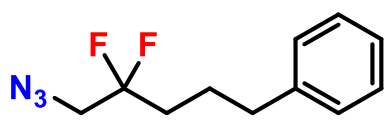

2j
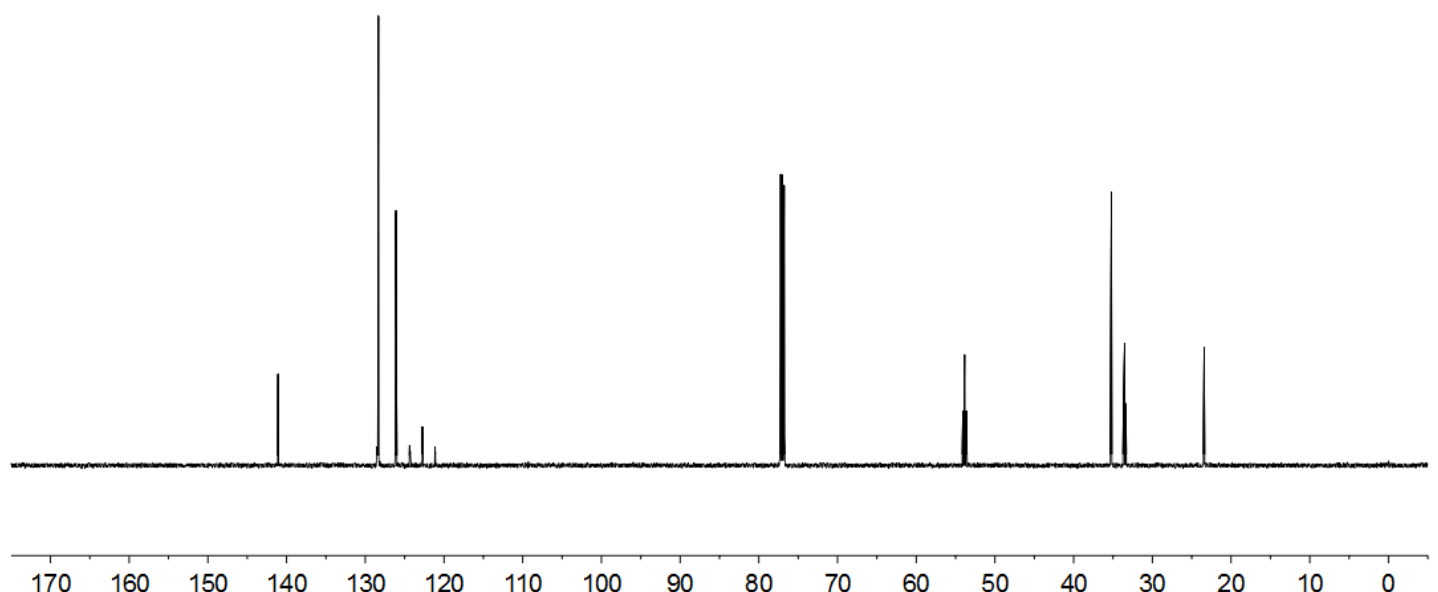

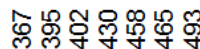

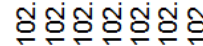<smiles>NCC(F)(F)CCCc1ccccc1</smiles>

2j

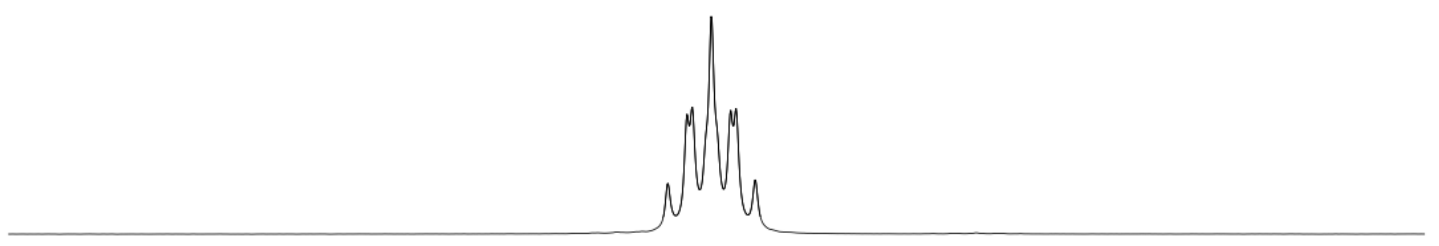

$\begin{array}{llllllllll}-101.5 & -101.7 & -101.9 & -102.1 & -102.3 & -102.5 & -102.7 & -102.9 & -103.1 & -103.3\end{array}$ 


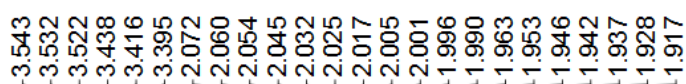

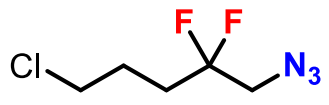

$2 k$

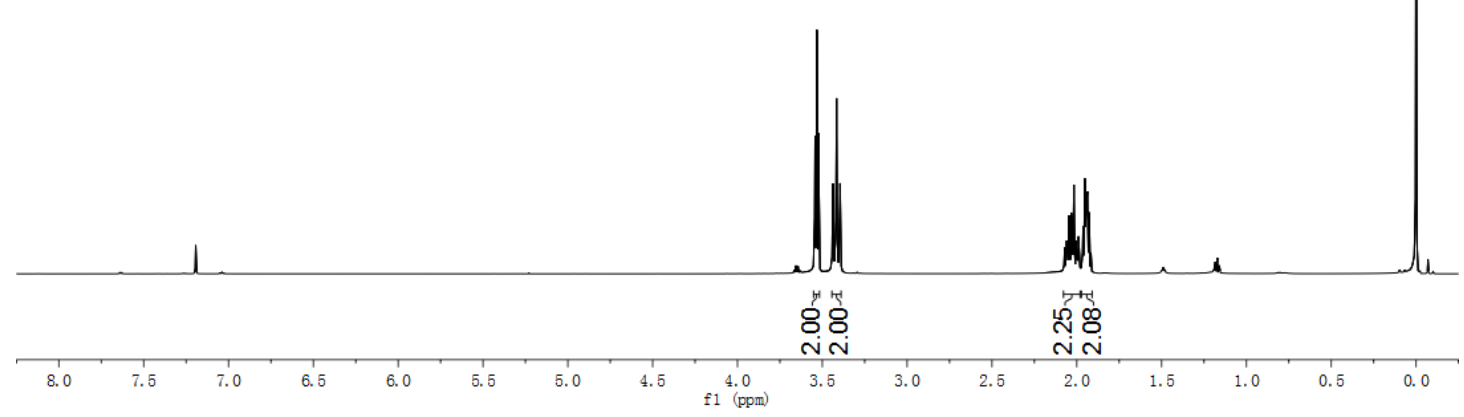

ำ

ปู่สำ

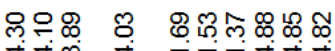

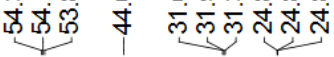

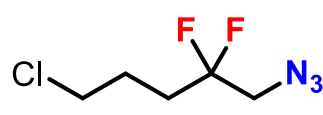

2k

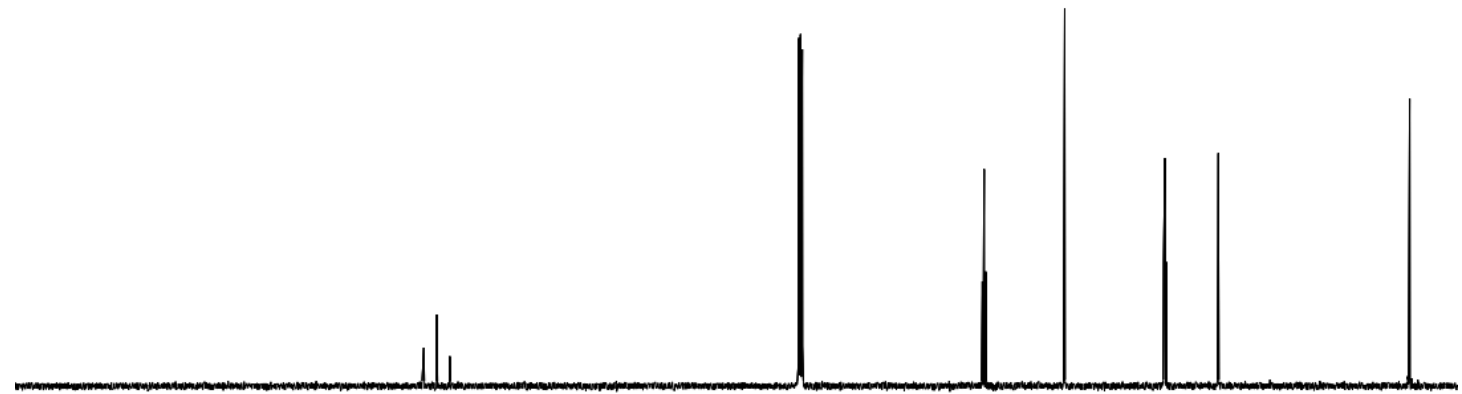

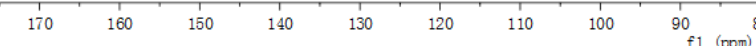

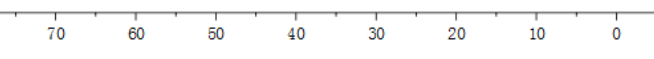




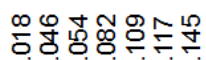

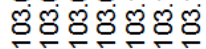 \\ 닌}

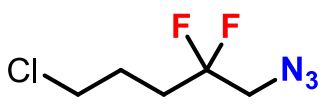

$2 k$

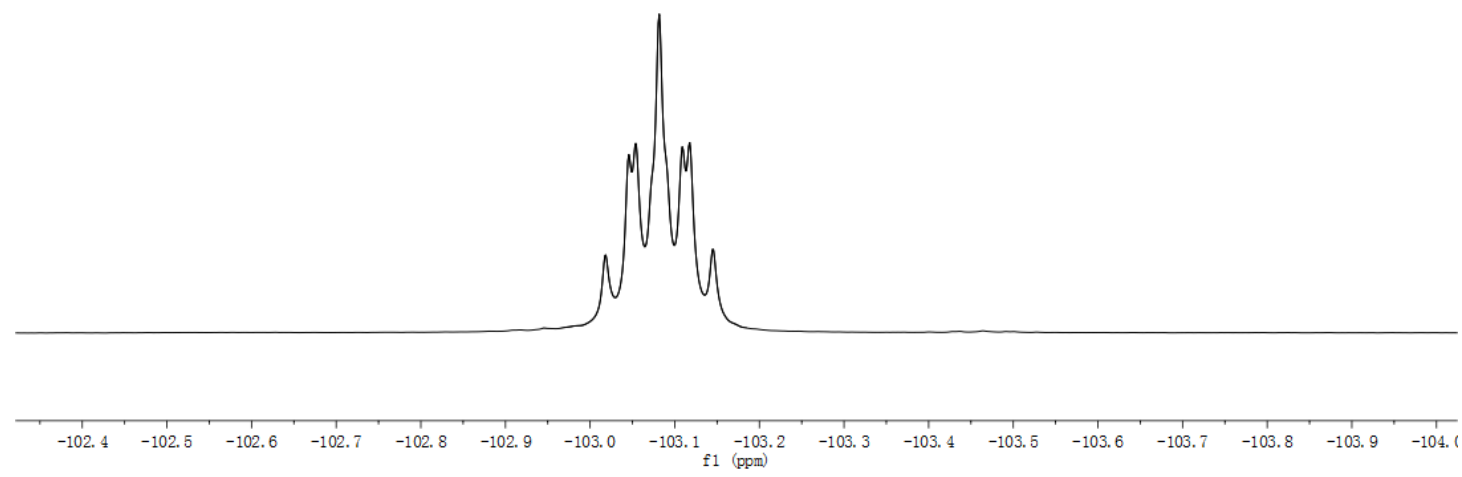

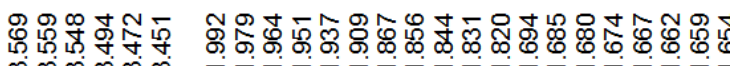

पบएँ

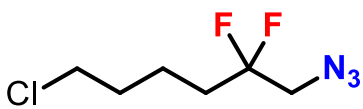

2I

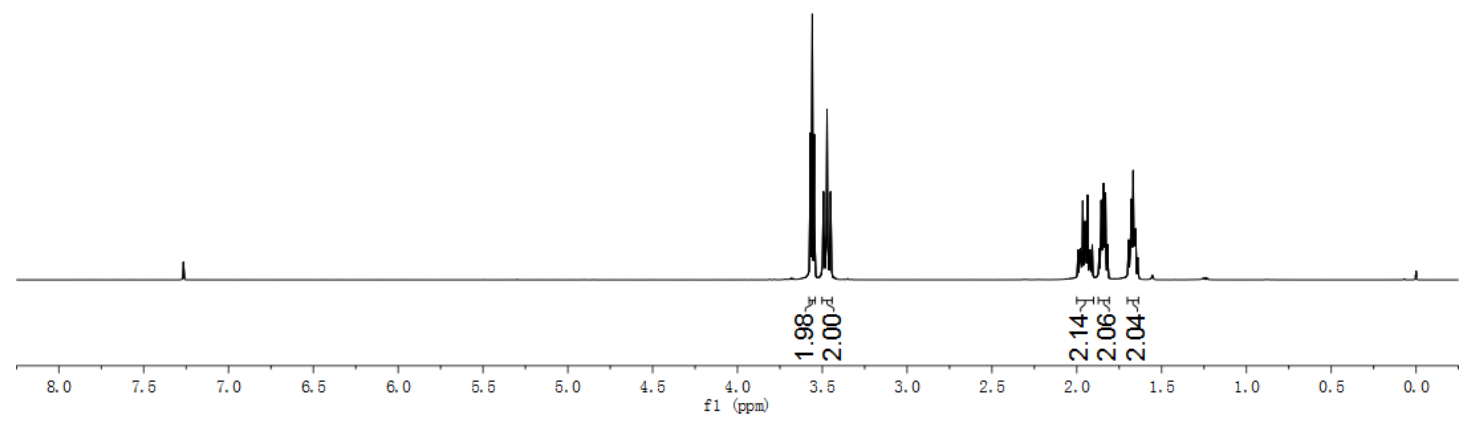




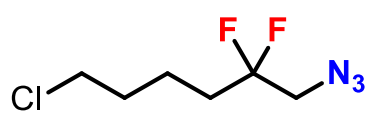

2I
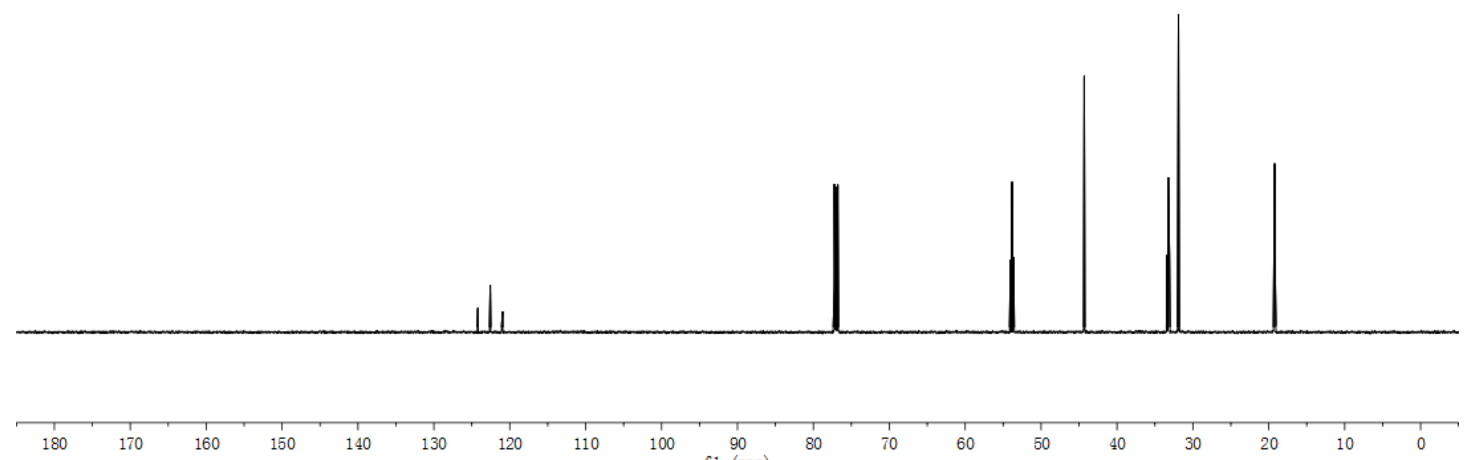

f1 $\stackrel{90}{(\mathrm{ppm})}$

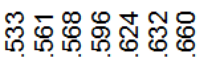

ปن

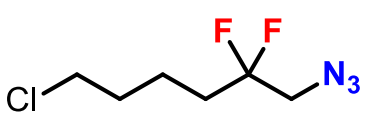

2I

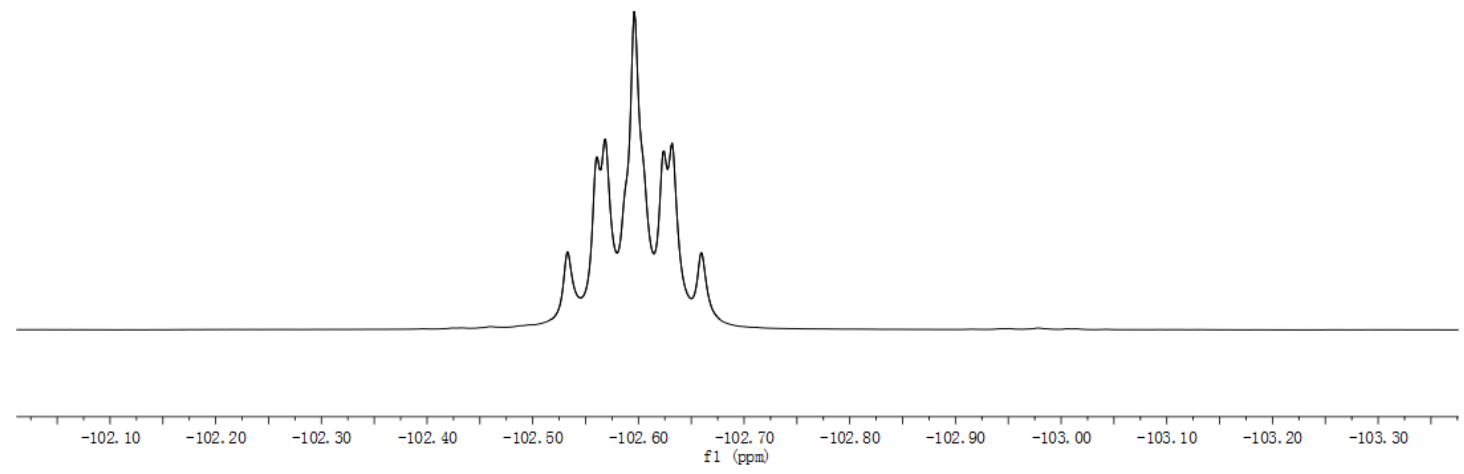




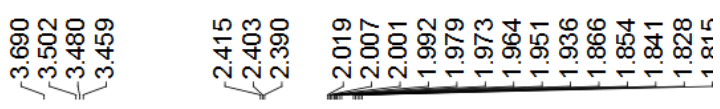

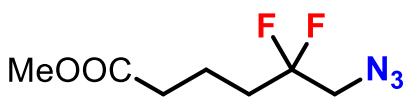

$2 m$

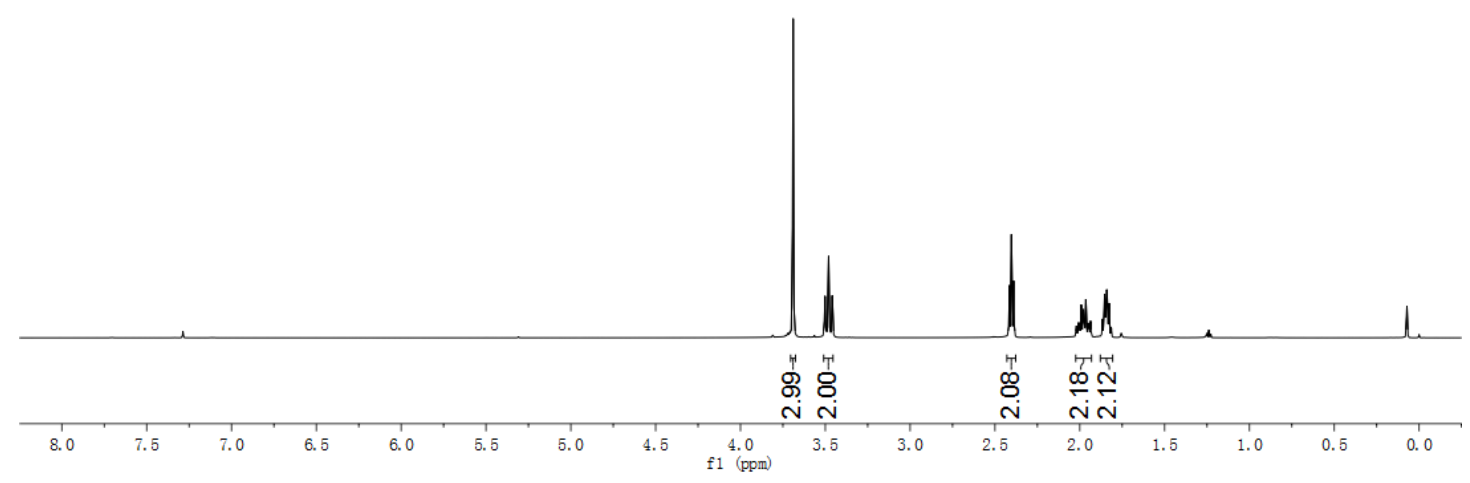

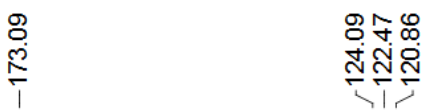

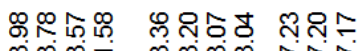

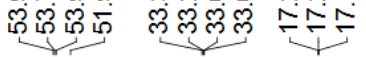

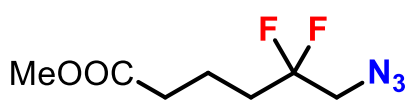

$2 m$

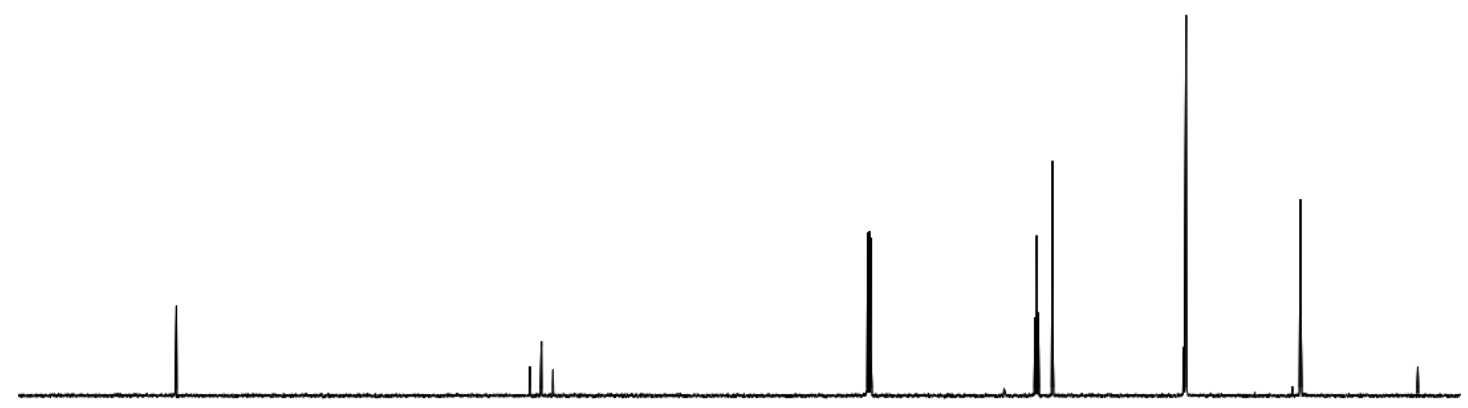

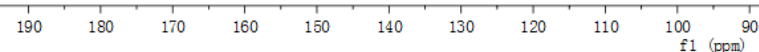




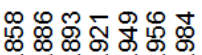

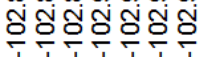

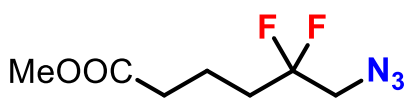

$2 m$

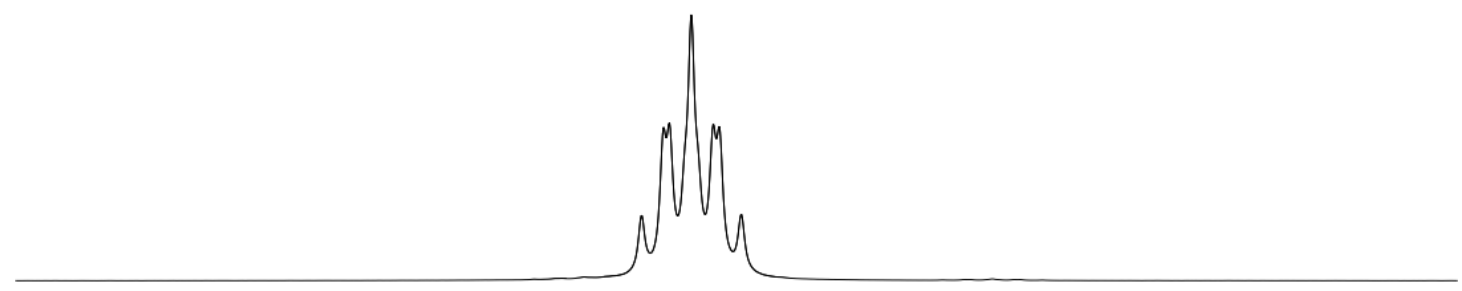

\begin{tabular}{lllllllllllllllllllll}
\hline-102.1 & -102.2 & -102.3 & -102.4 & -102.5 & -102.6 & -102.7 & -102.8 & -102.9 & -103.0 & -103.1 & -103.2 & -103.3 & -103.4 & -103.5 & -103.6 & -103.7 & -103.8 &
\end{tabular}

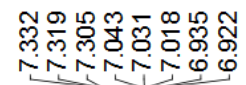

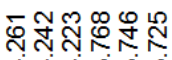

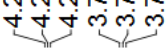

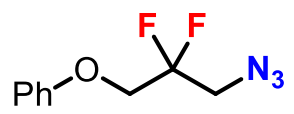

2n

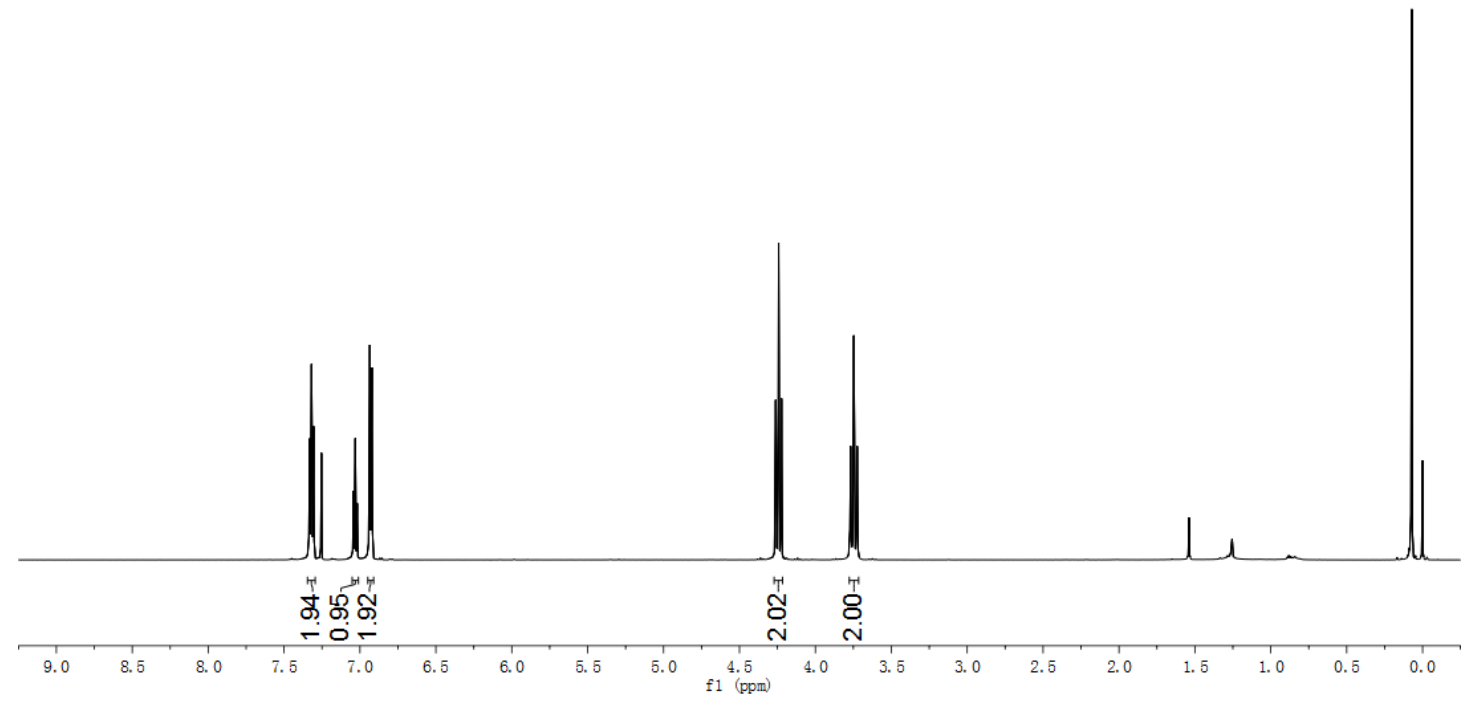



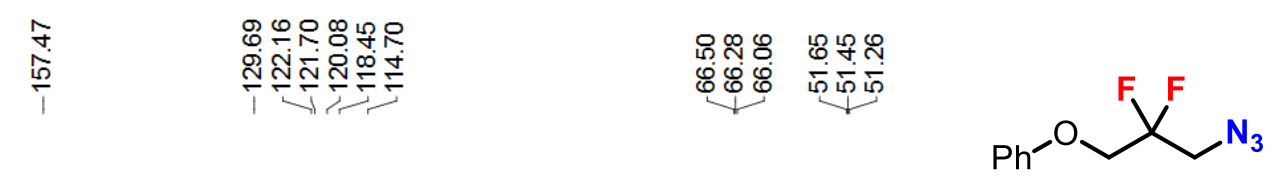

2n
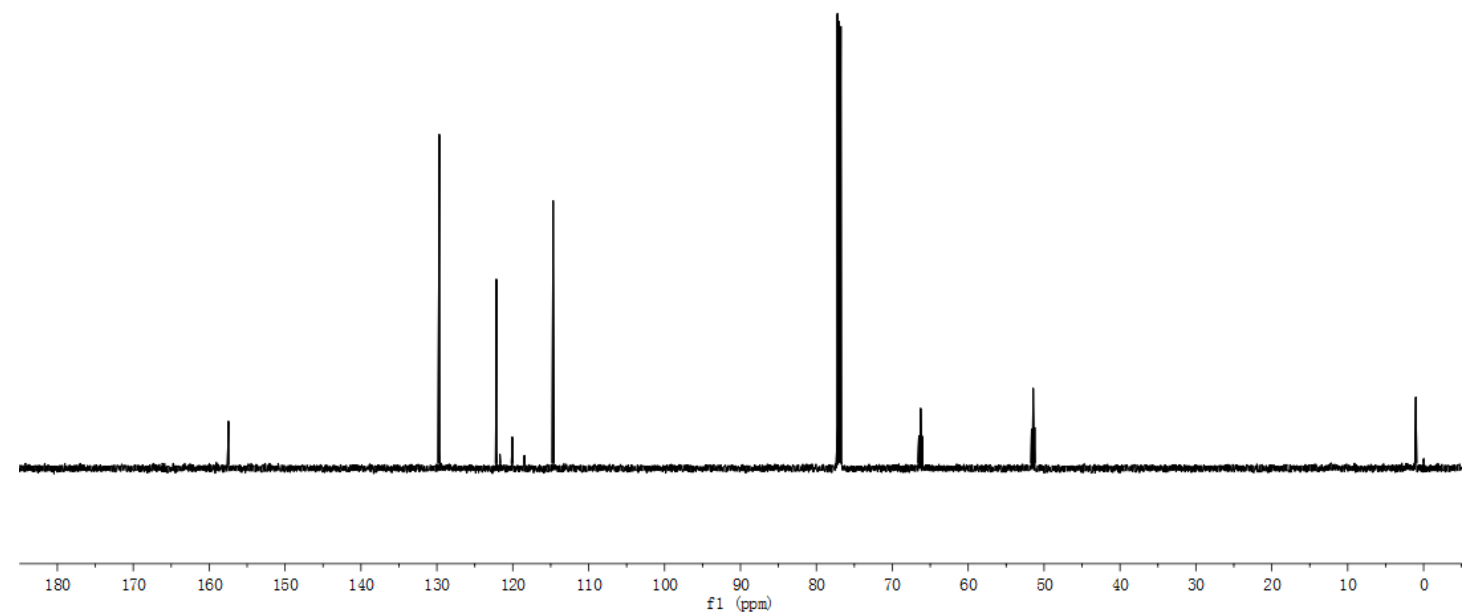

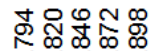

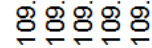

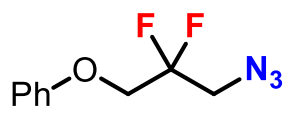

2n

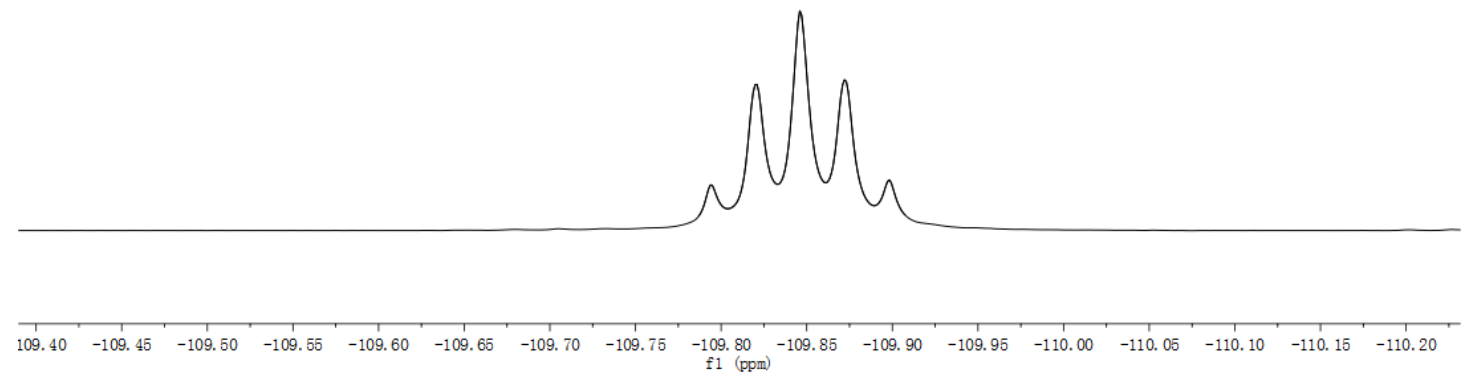




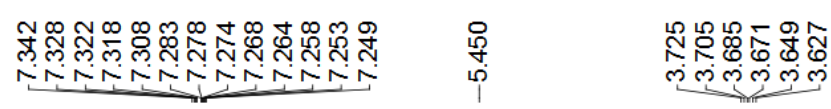

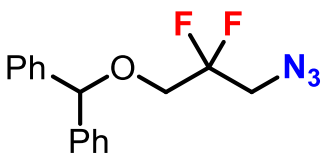

20

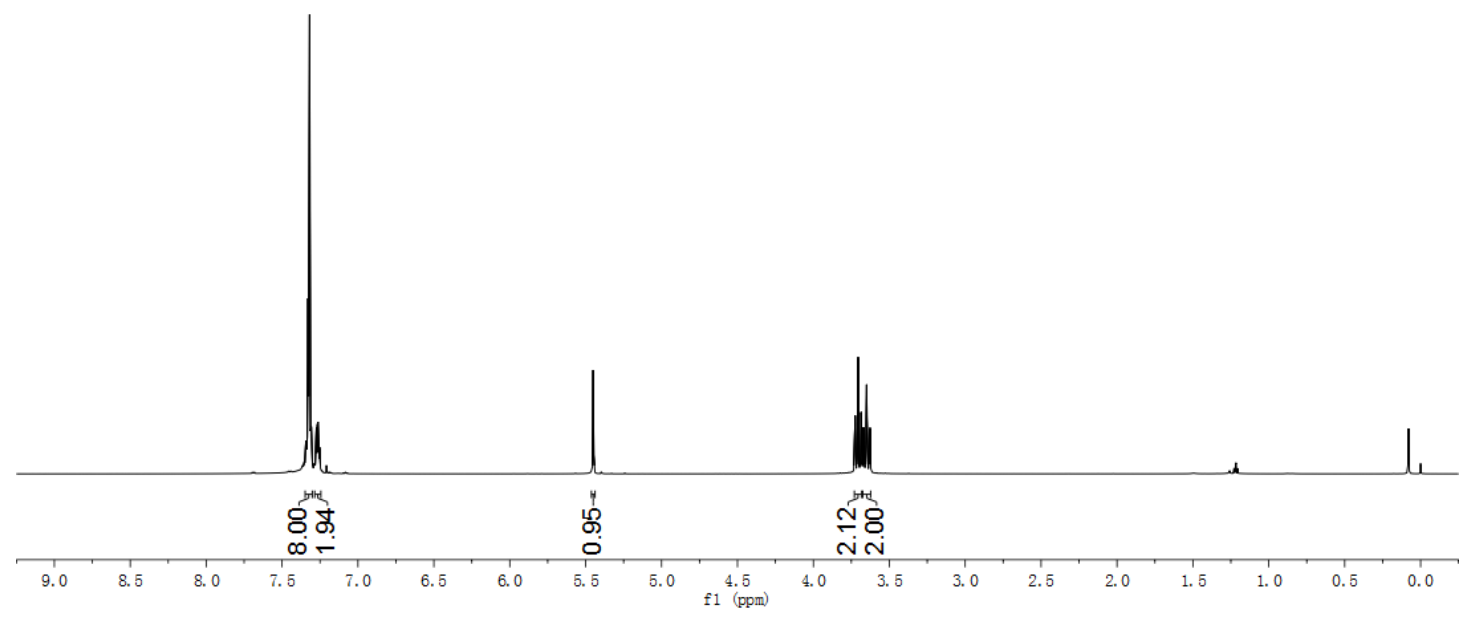

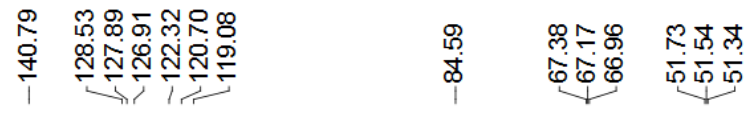

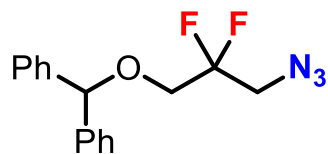

20

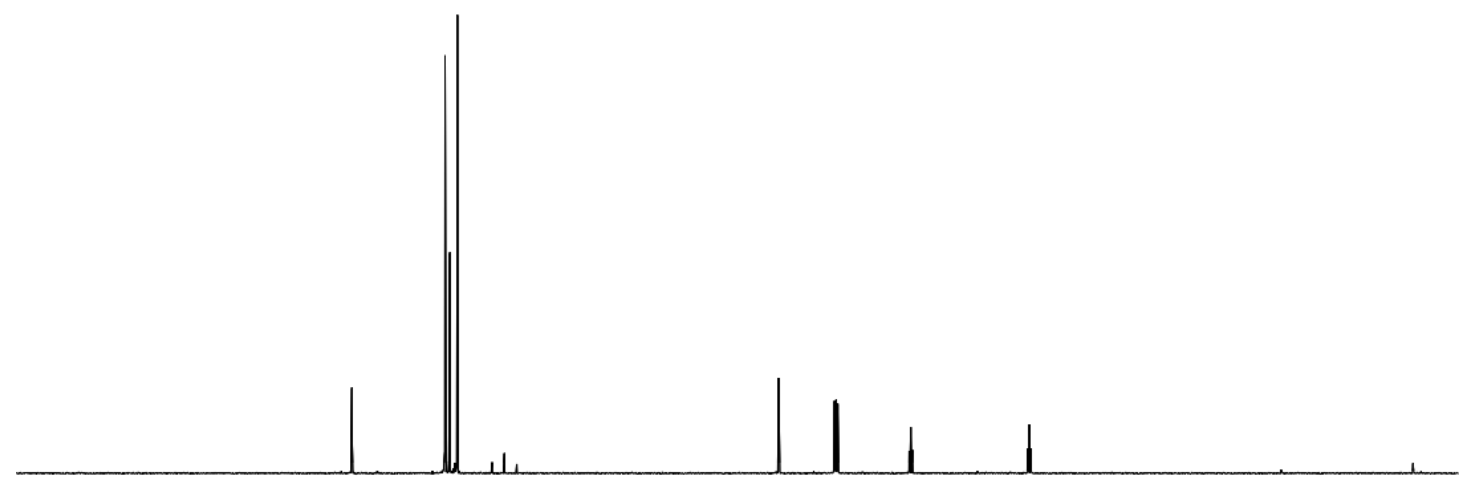

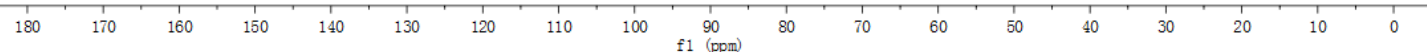




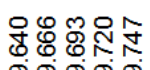

훙훙ㅇㅇㅁ

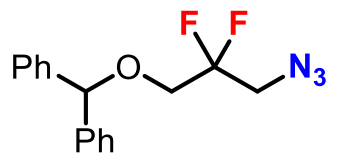

20
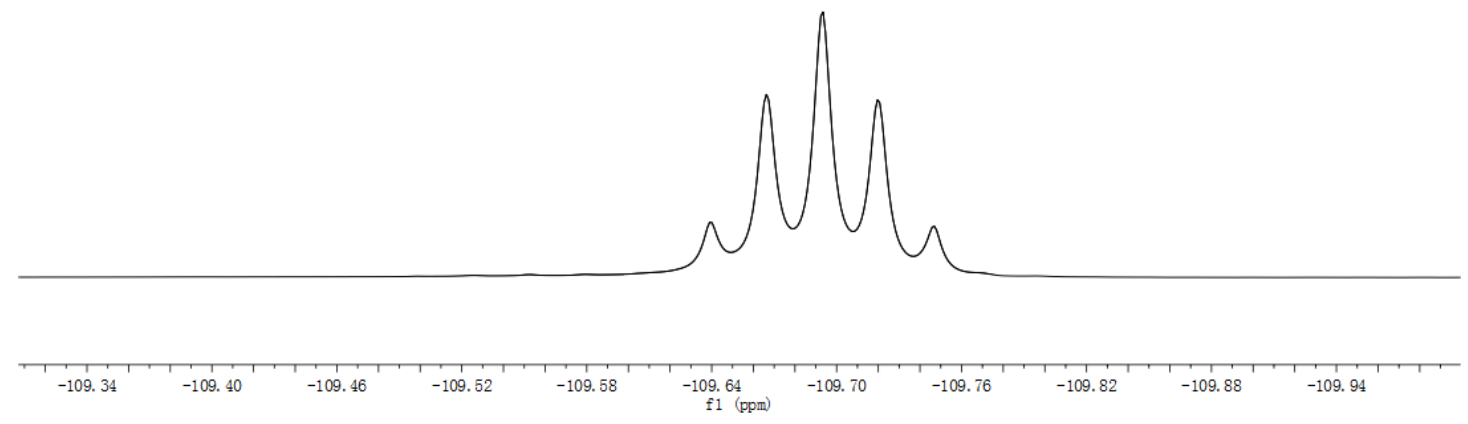

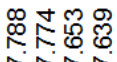

些资

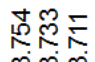

लंल

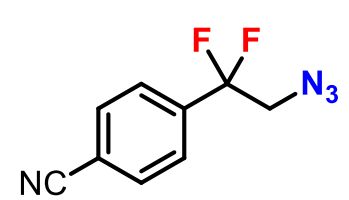

$2 p$

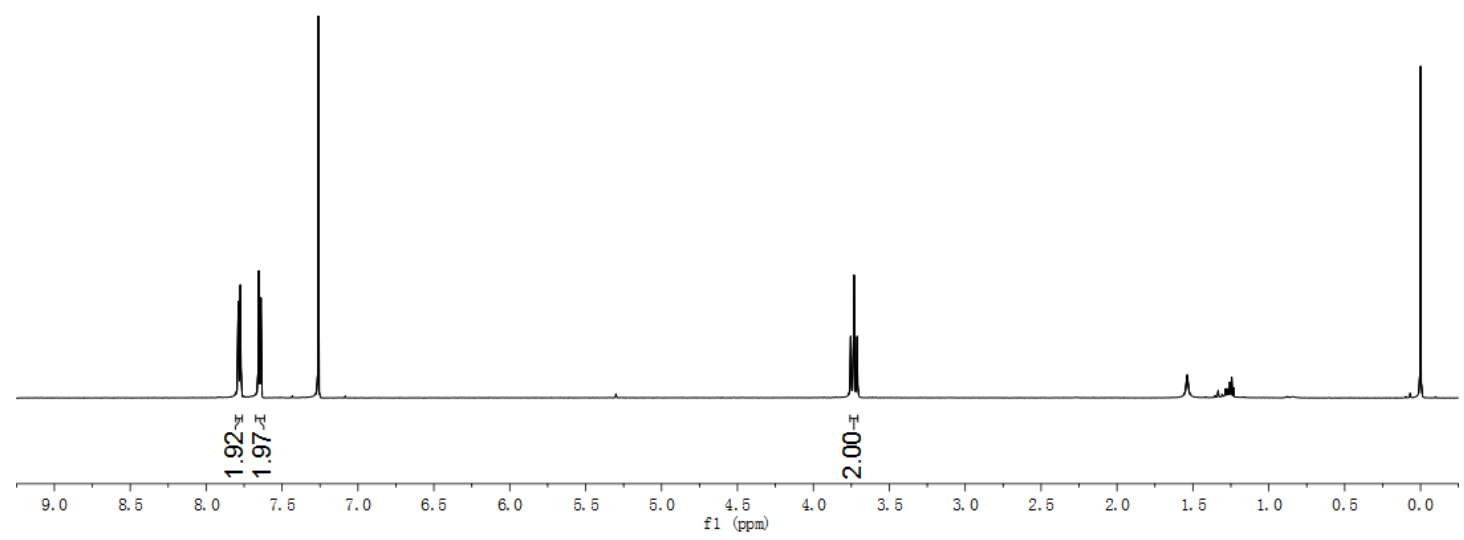




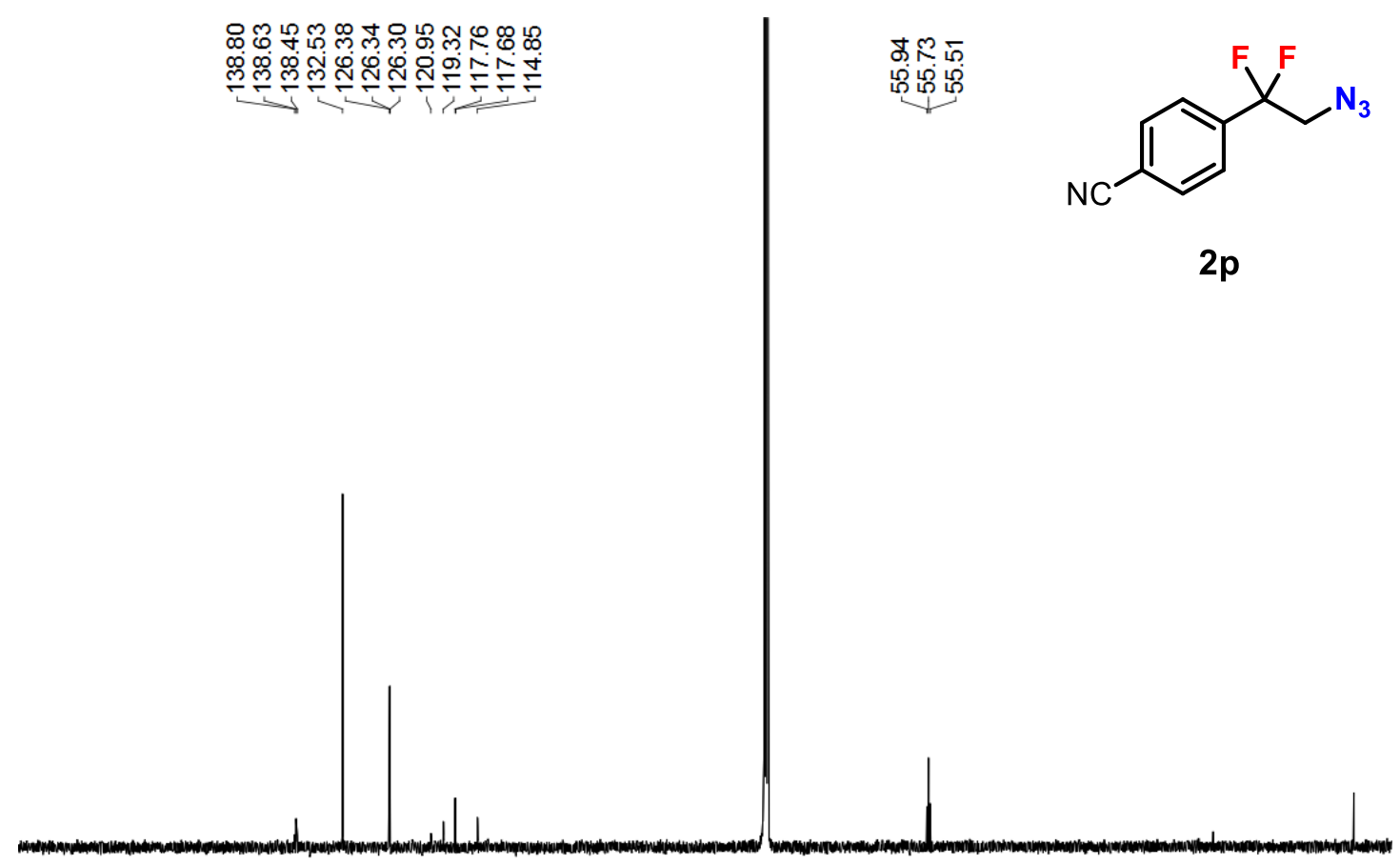

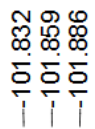<smiles>N#Cc1ccc(C(F)(F)CN)cc1</smiles>

$2 p$

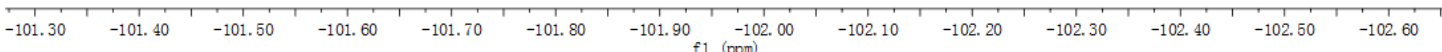




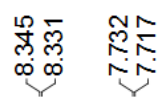

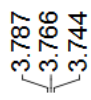

(N)

$2 q$

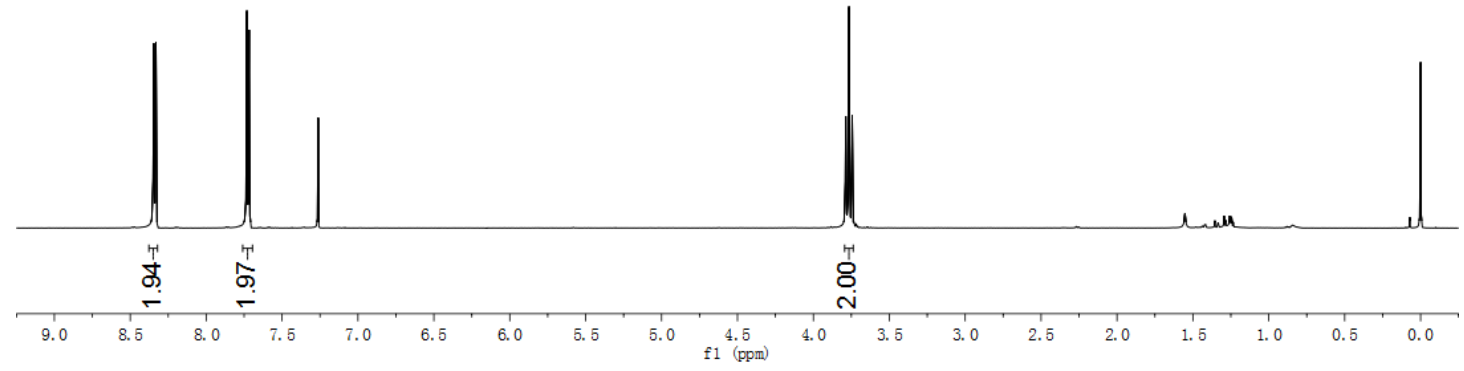

กิ พํํ

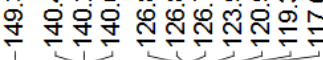

里点

莡望皆
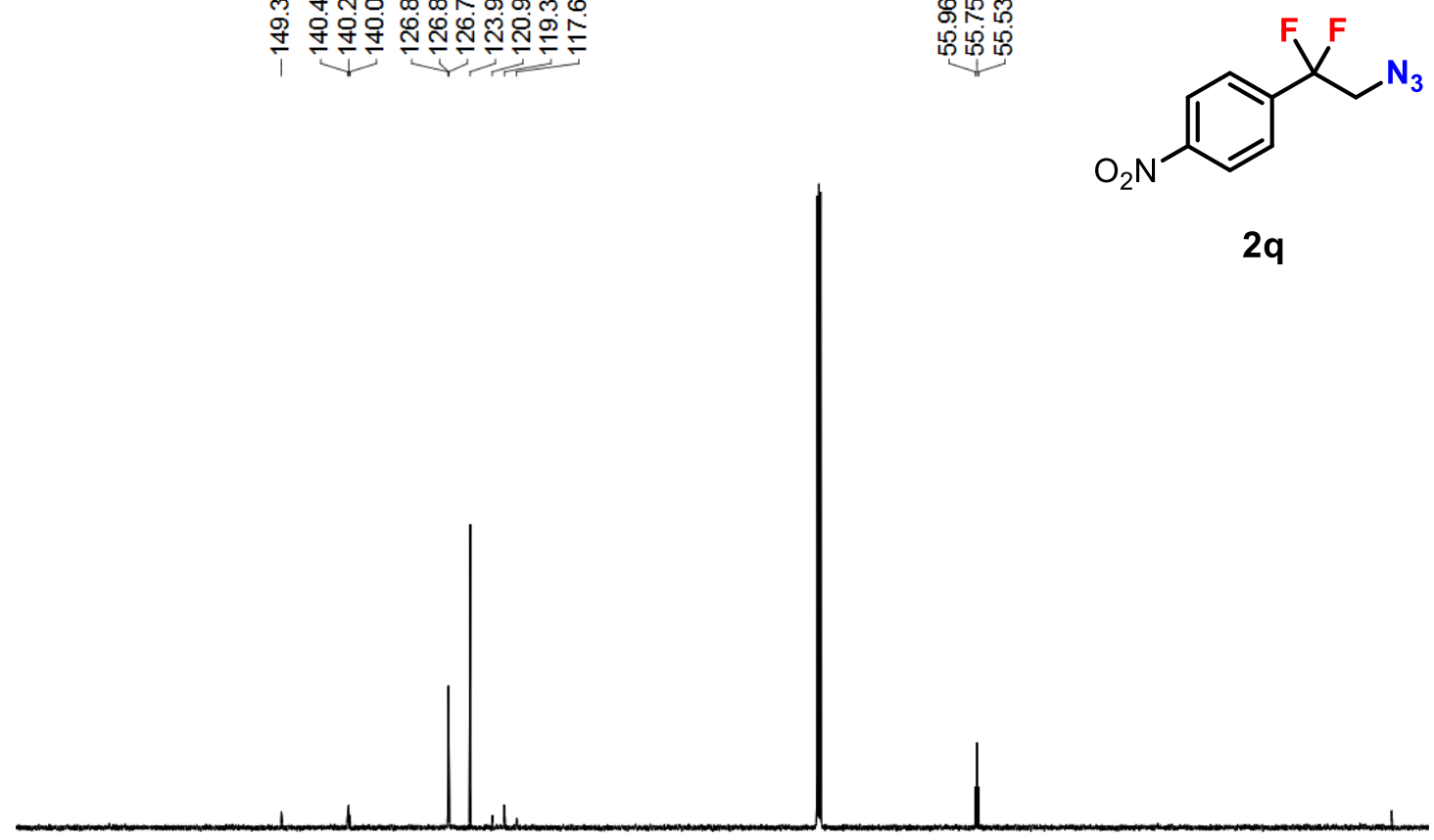

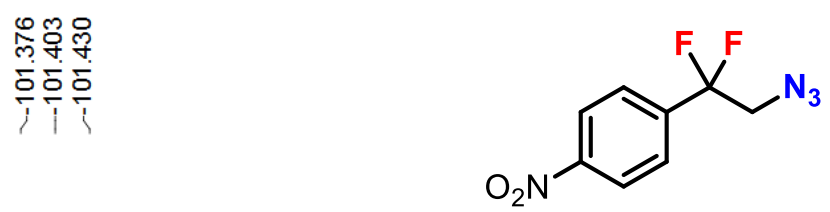

$2 q$

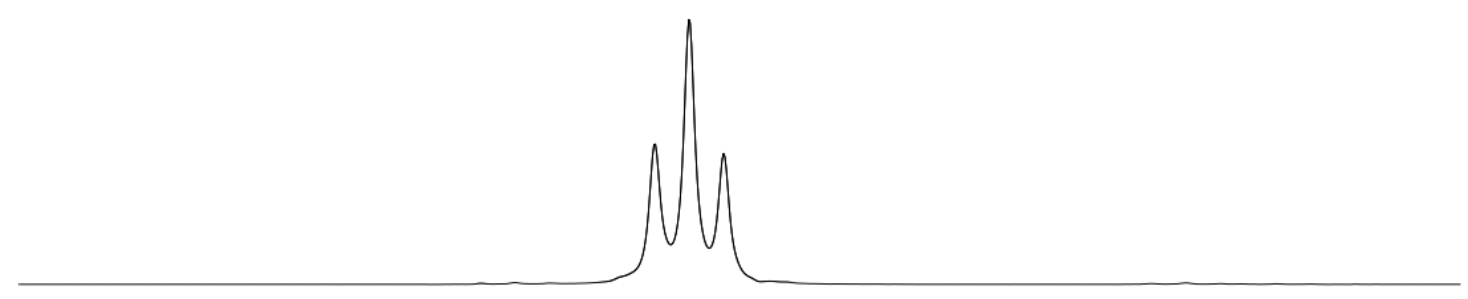

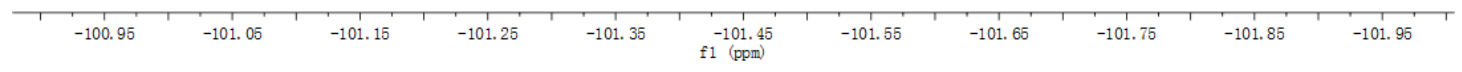

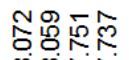

禹舟

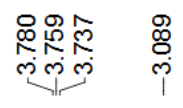
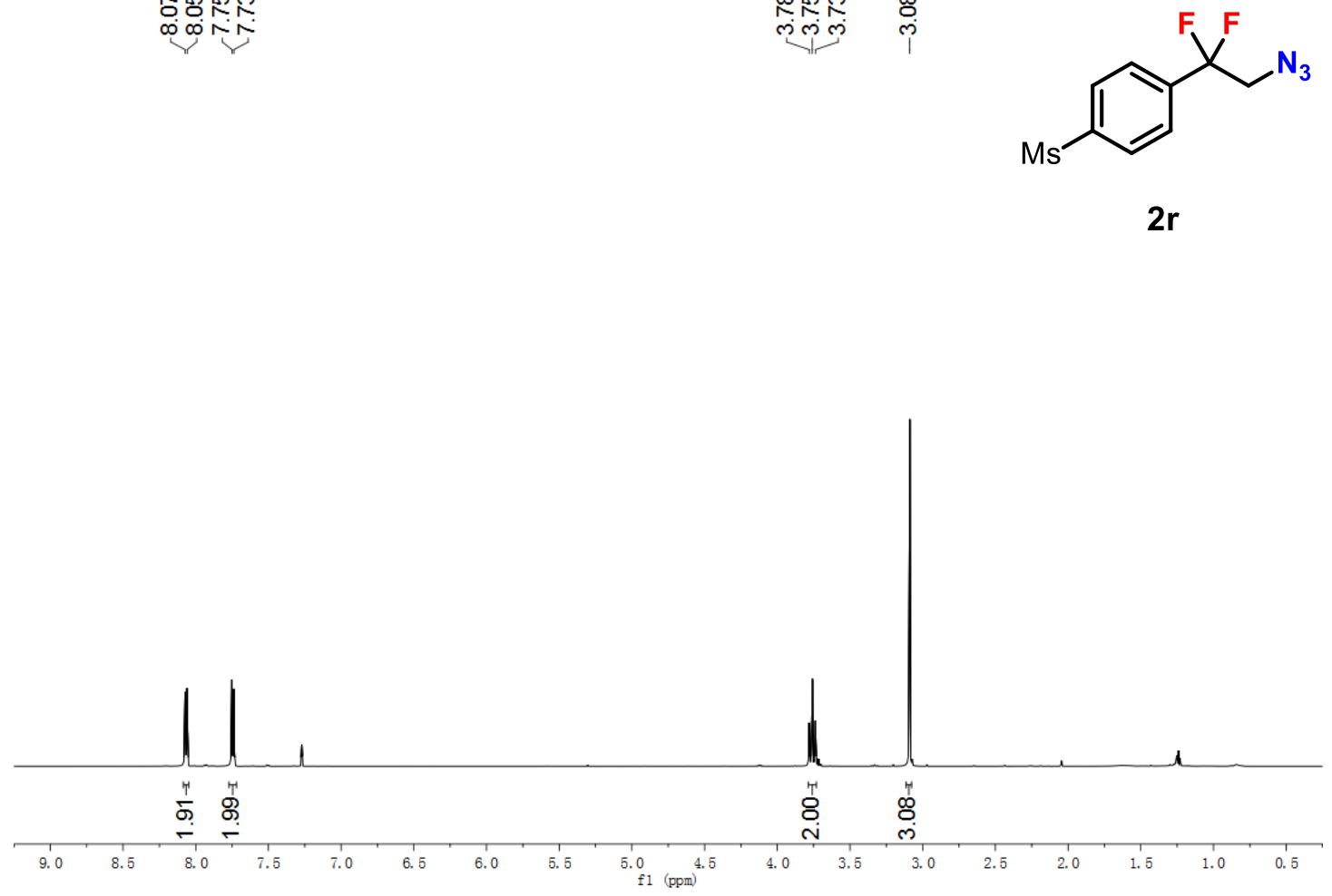

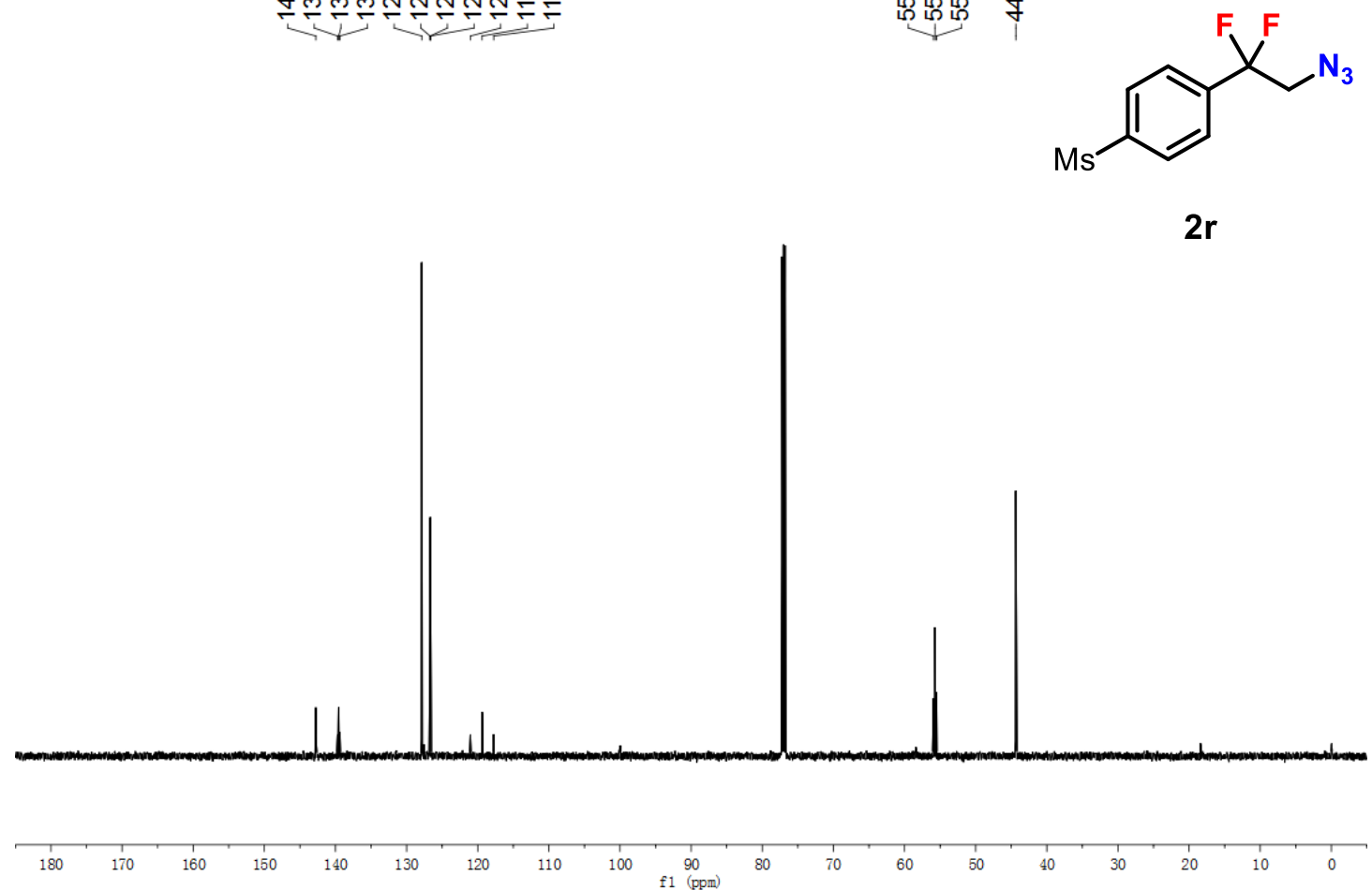

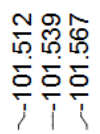
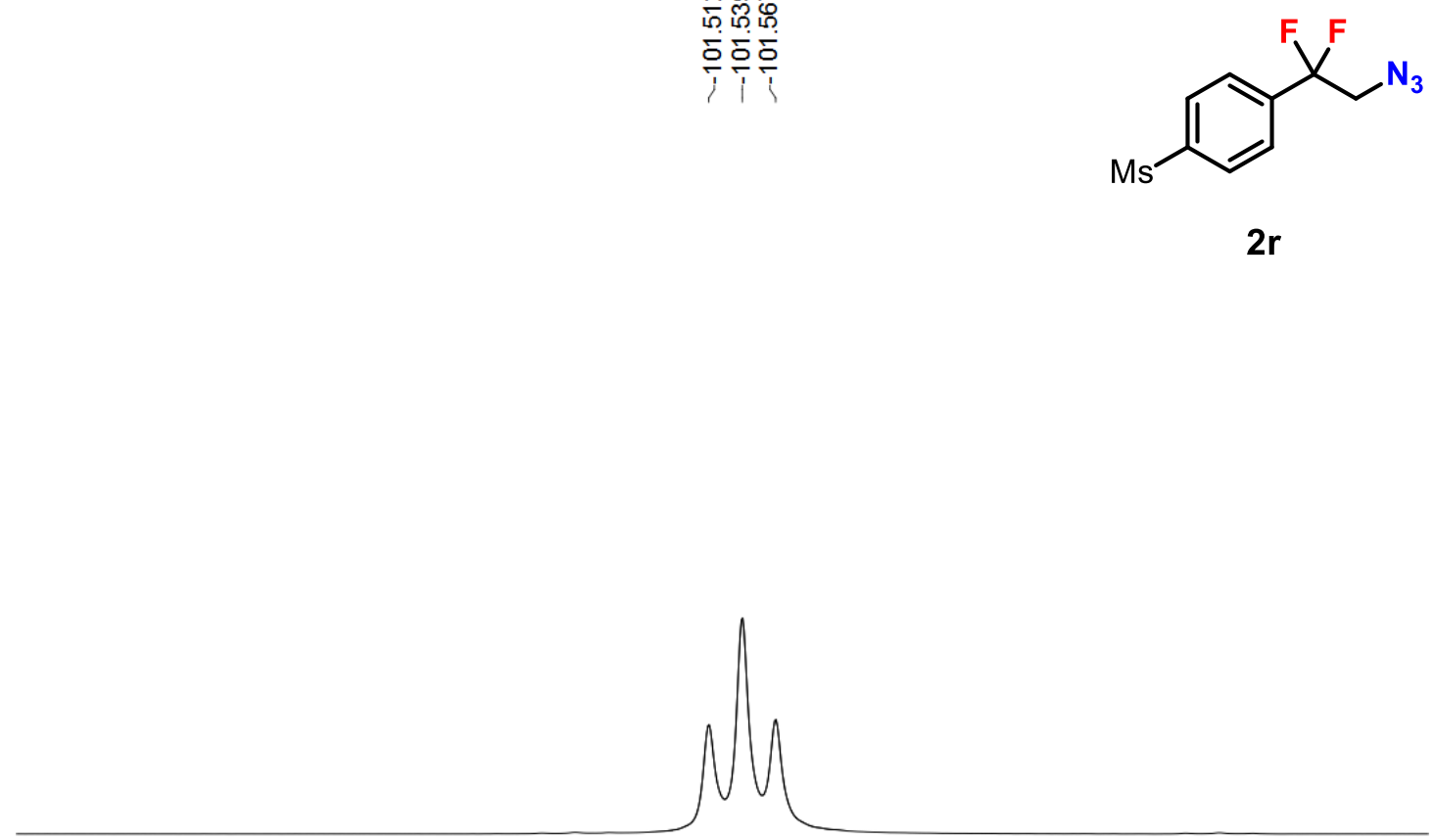


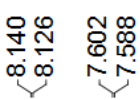

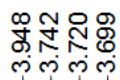

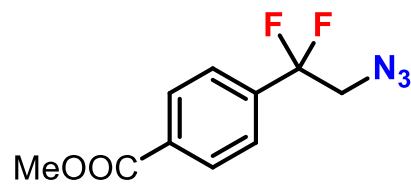

$2 s$

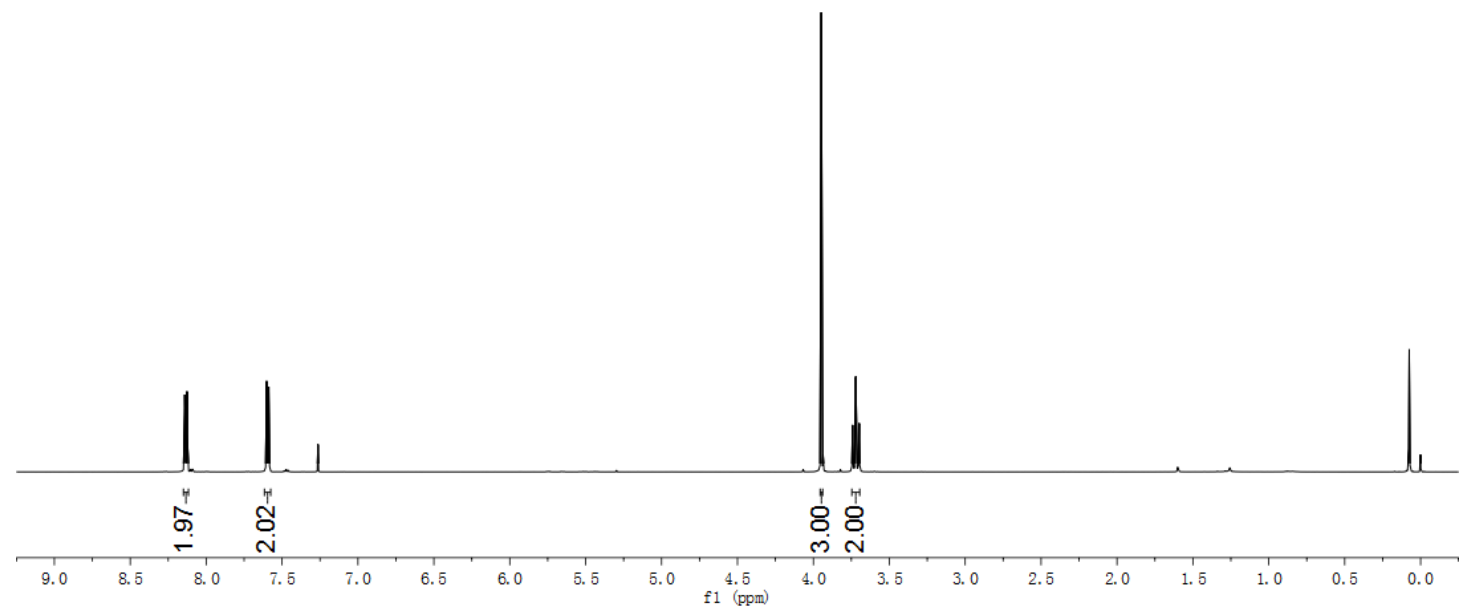

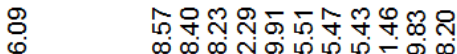

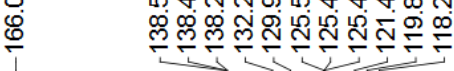

웅용요

นึำน
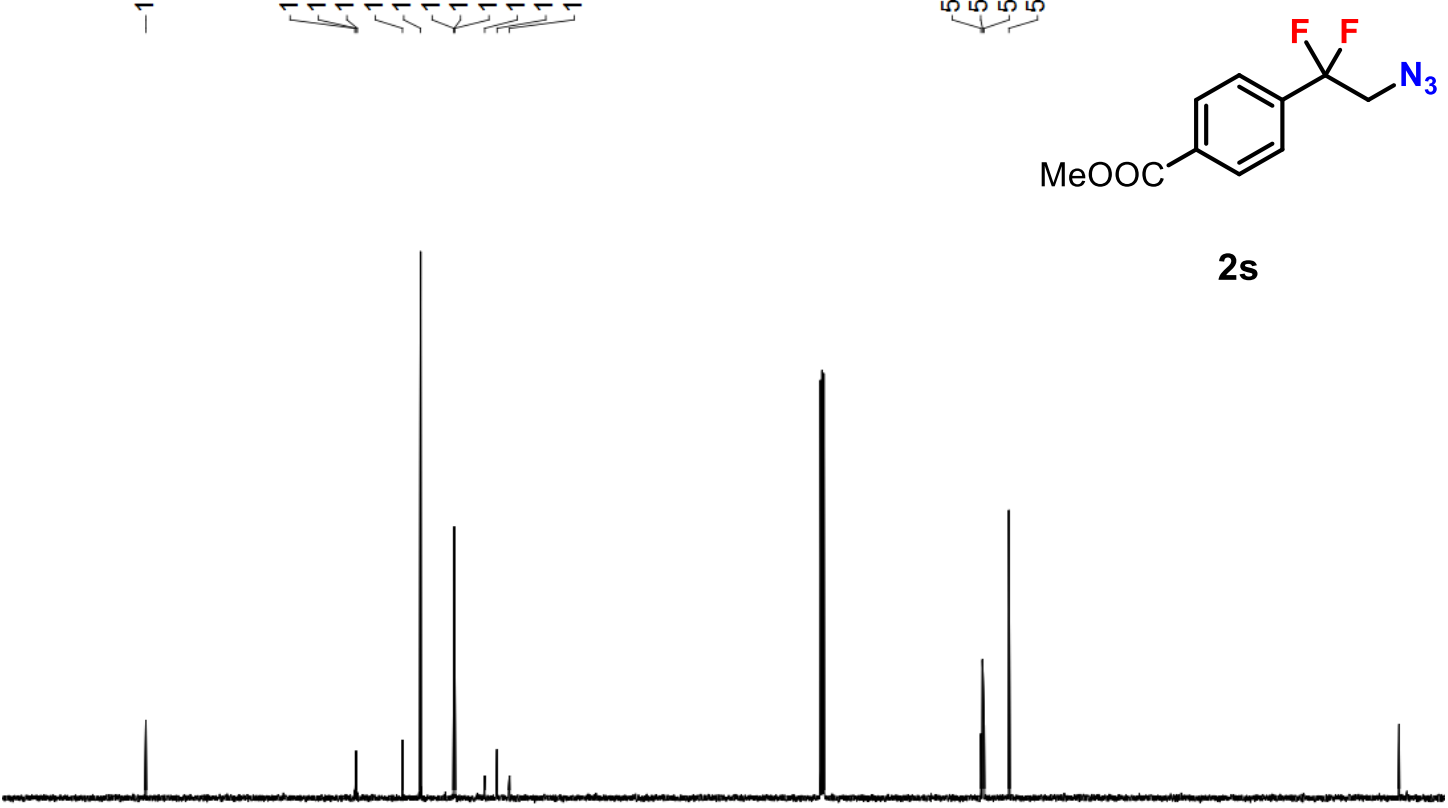

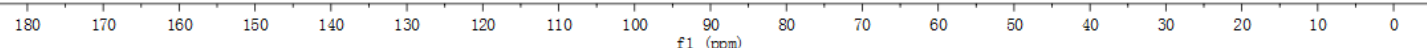




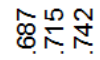 \\ 흐흐므 \\ 훈휘운}<smiles>COC(=O)c1ccc(C(F)(F)CN)cc1</smiles>

2s
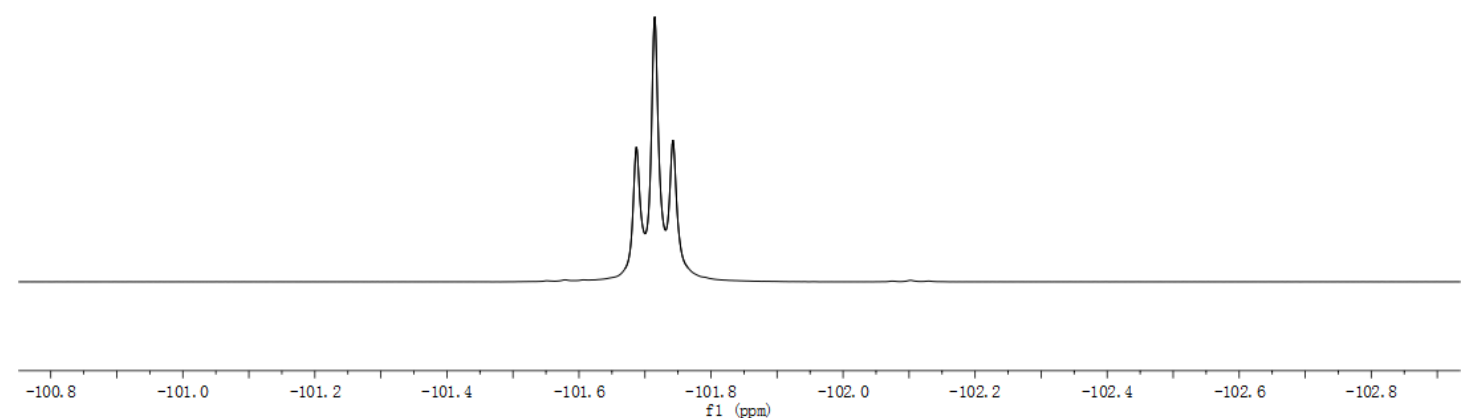

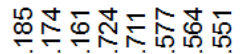

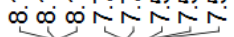

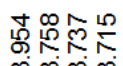

लंखिल

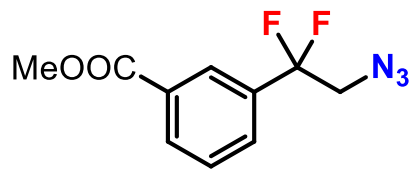

$2 t$

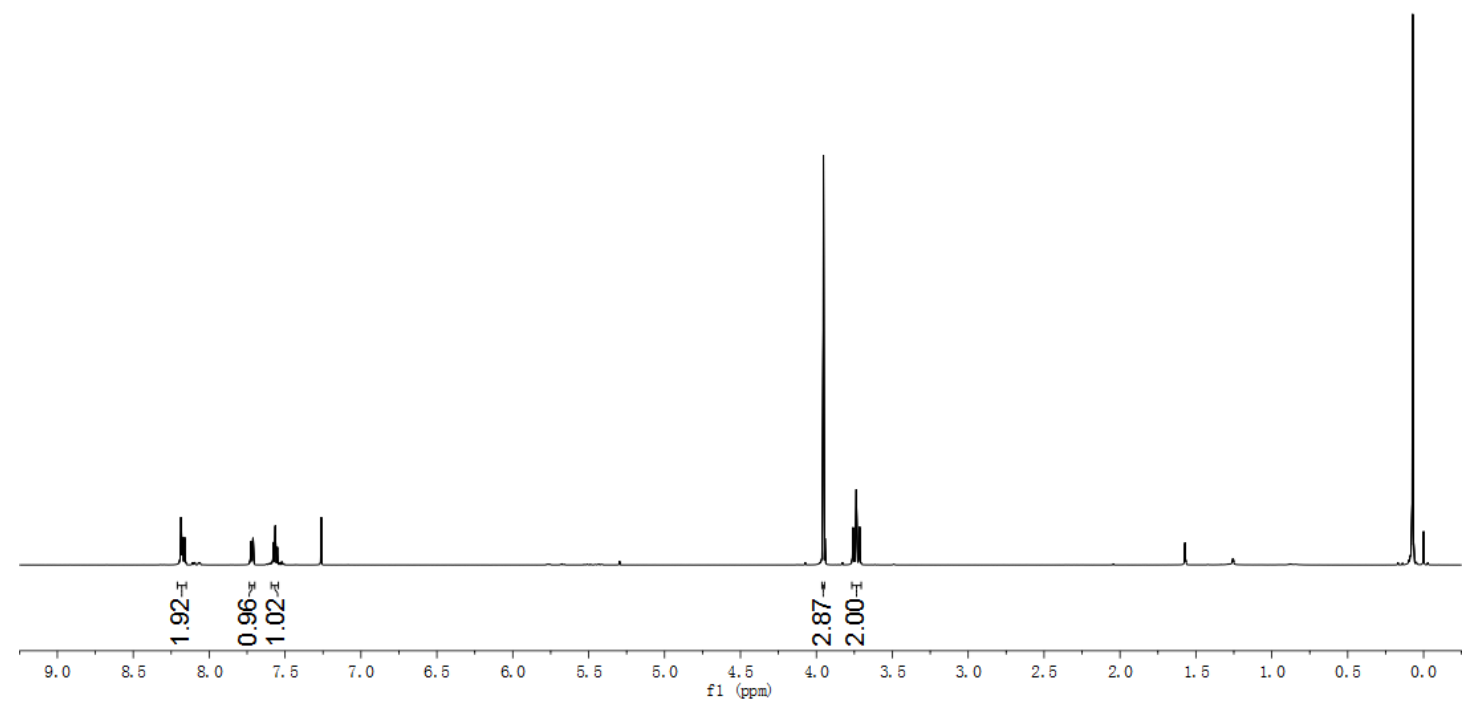


<smiles>COC(=O)c1cccc(C(F)(F)CN)c1</smiles>
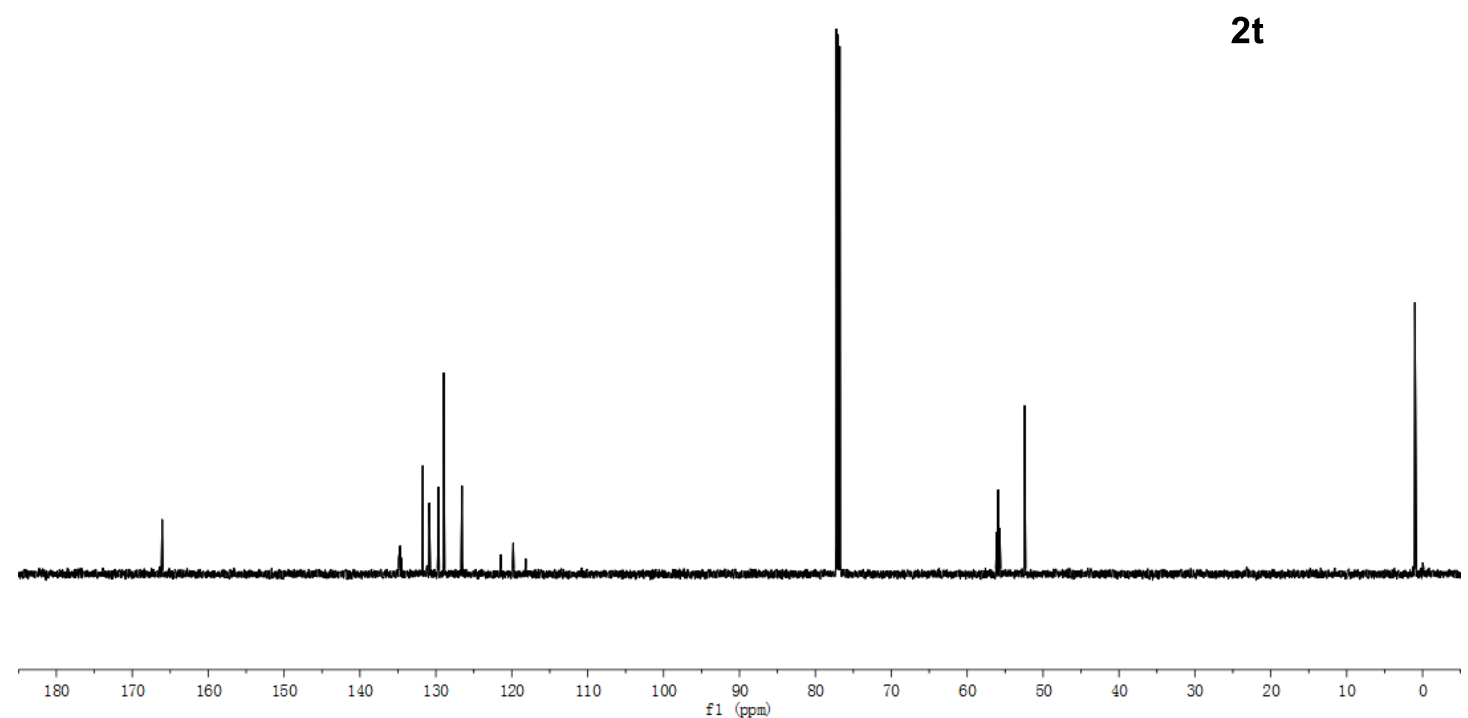

్ㅣㅁ음

흔흔<smiles>COC(=O)c1cccc(C(F)(F)CN)c1</smiles>

$2 t$

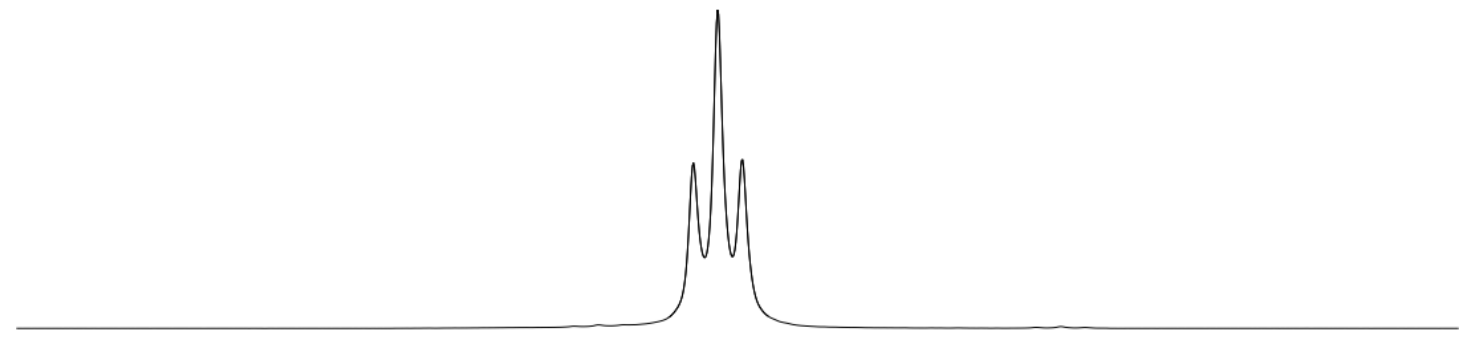

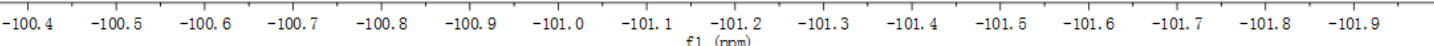




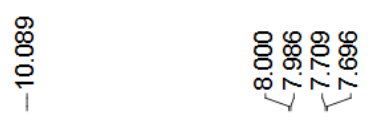

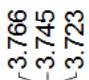

(NC)

$2 u$

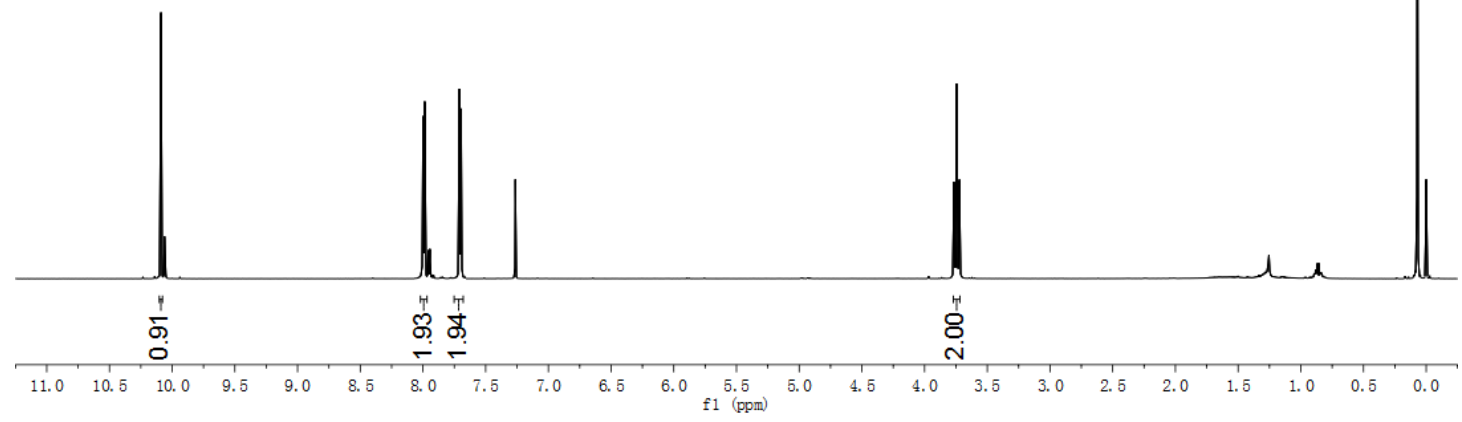

잉

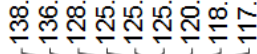

(2)

员花古<smiles>NCC(F)(F)c1ccc(C=O)cc1</smiles>

$2 u$

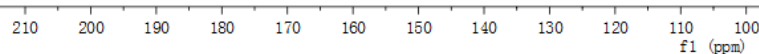




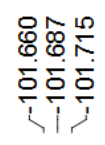

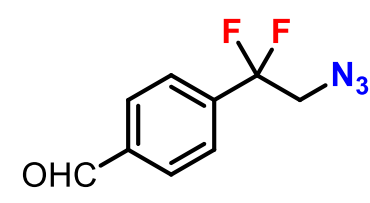

$2 u$
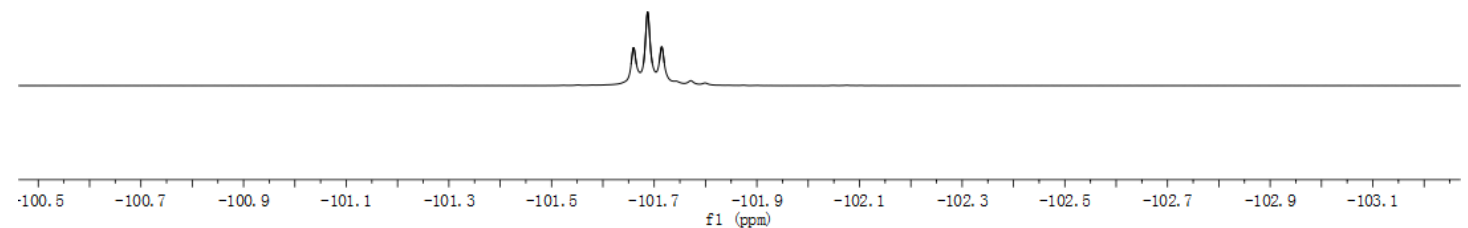

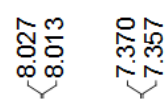

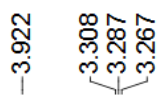

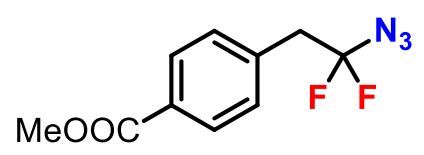

3s

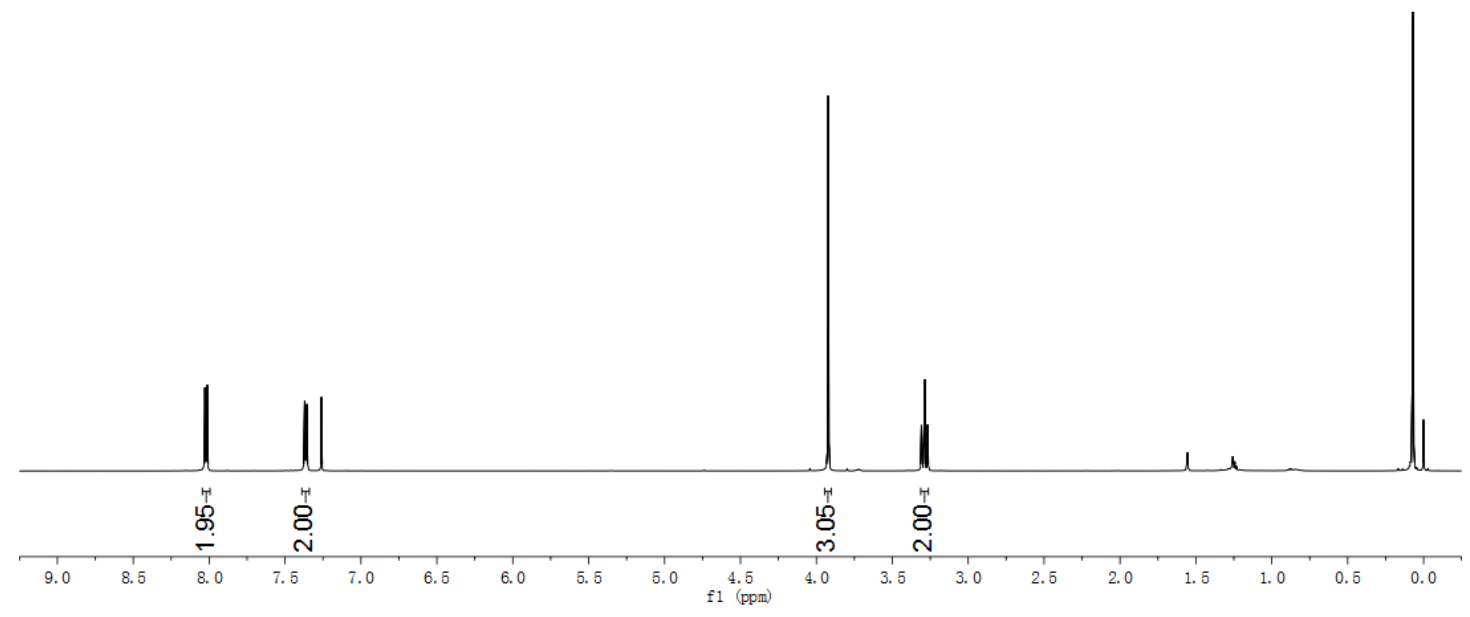



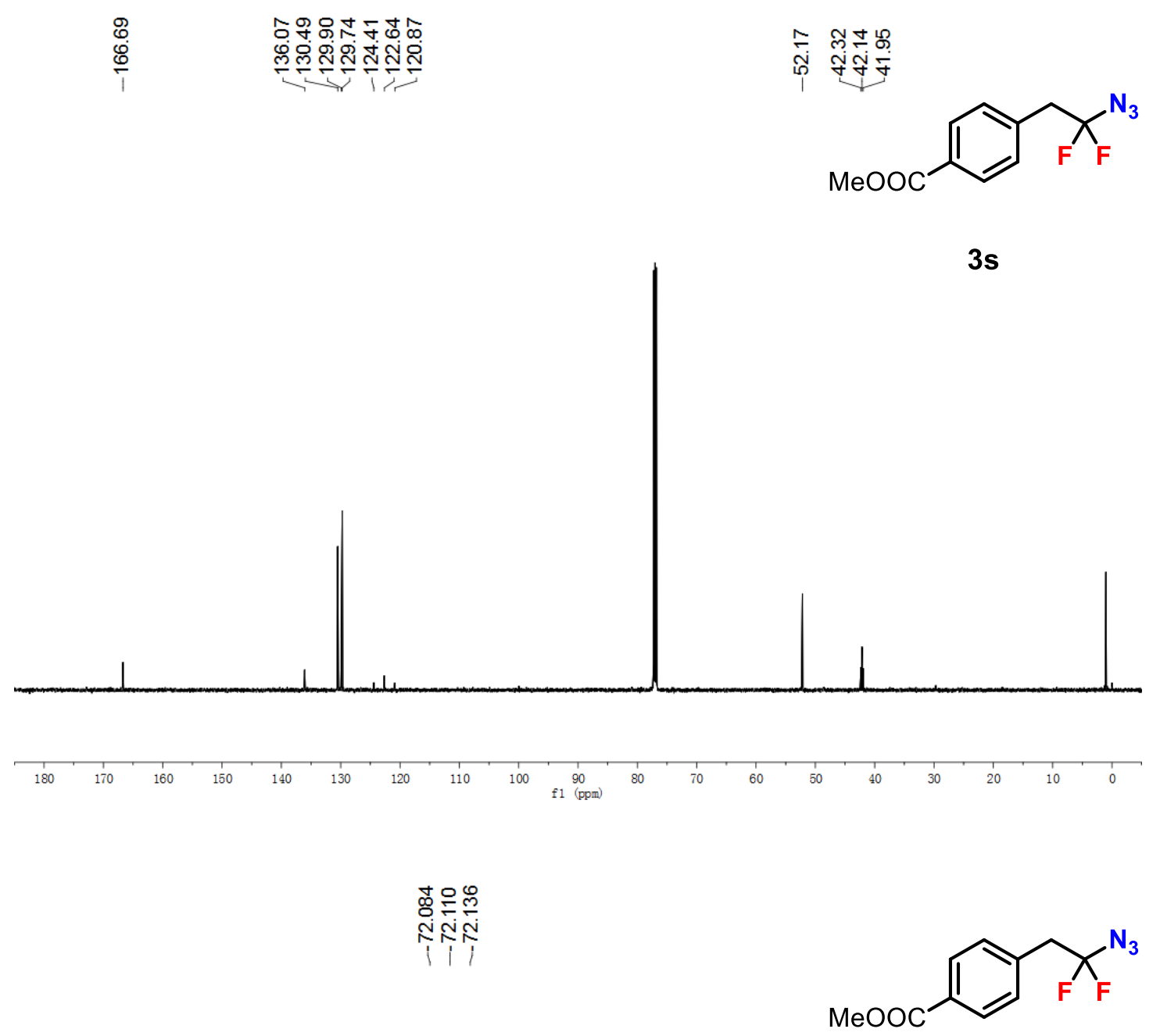

3s

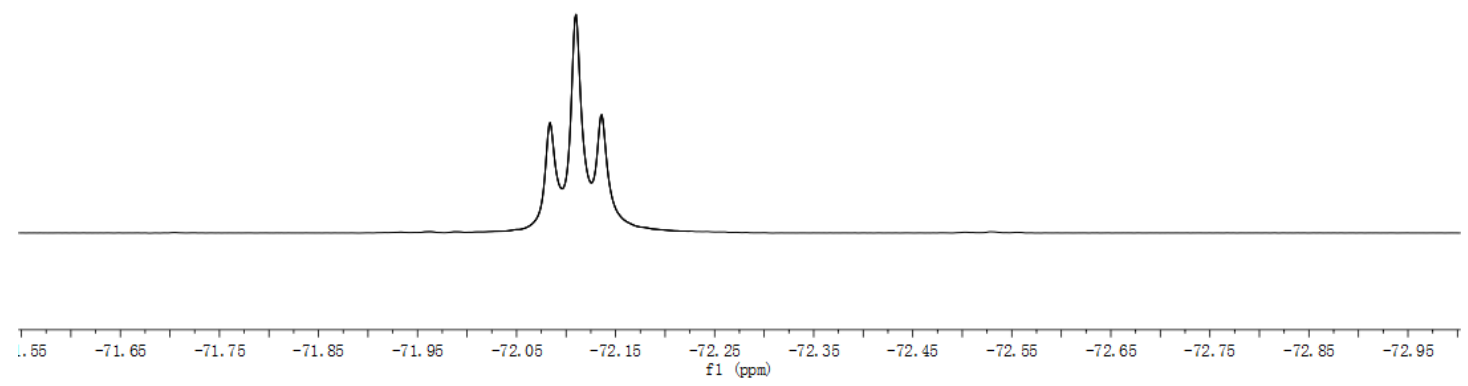




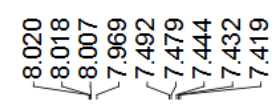

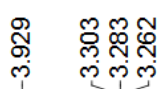

$\underbrace{\mathrm{MeOOC}}_{F} \mathrm{~F}_{\mathrm{F}}^{\mathrm{N}_{3}}$

$3 t$

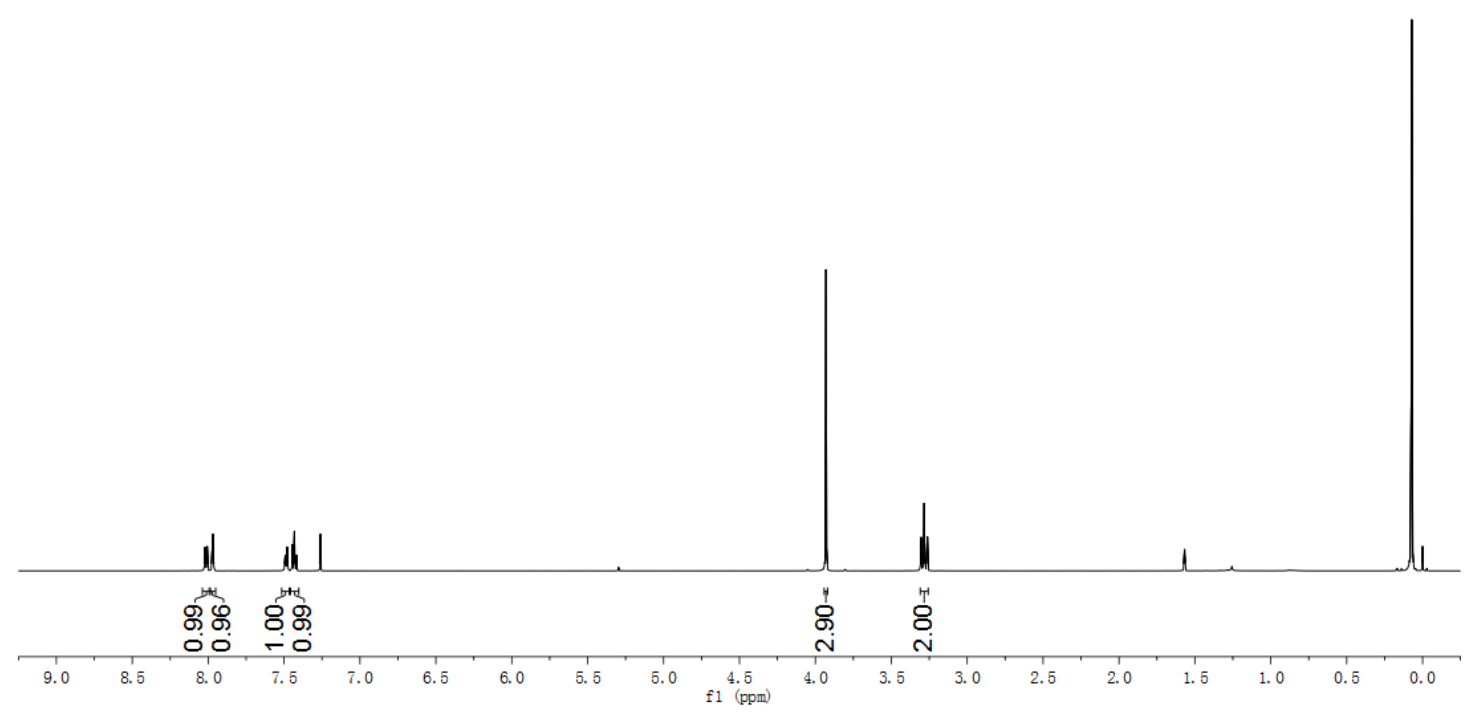

$\begin{array}{ll}0 & 0 \\ 0 & 0\end{array}$

กิ่

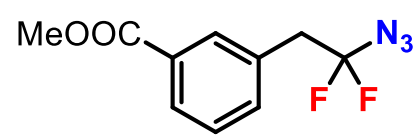

$3 t$

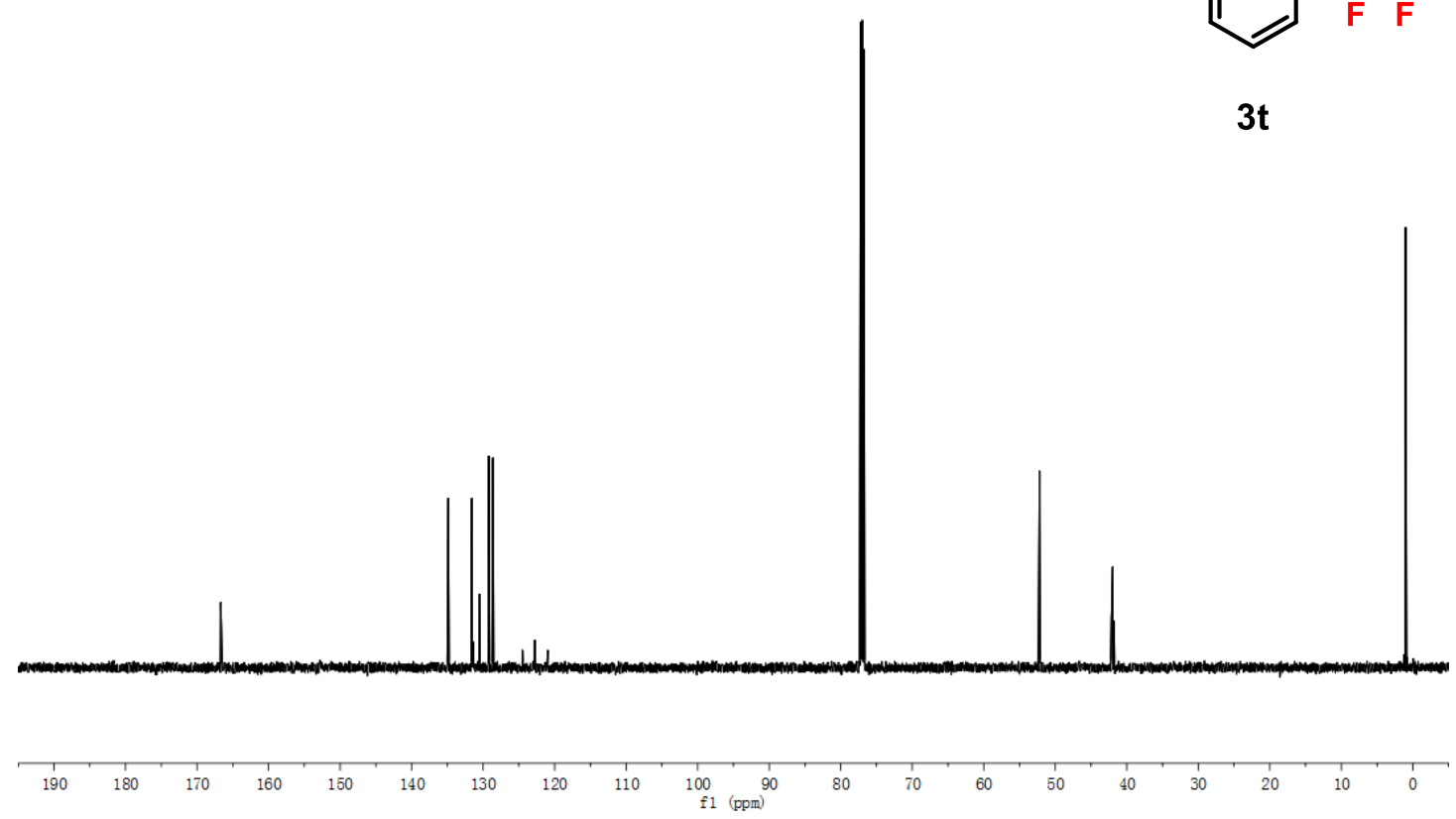




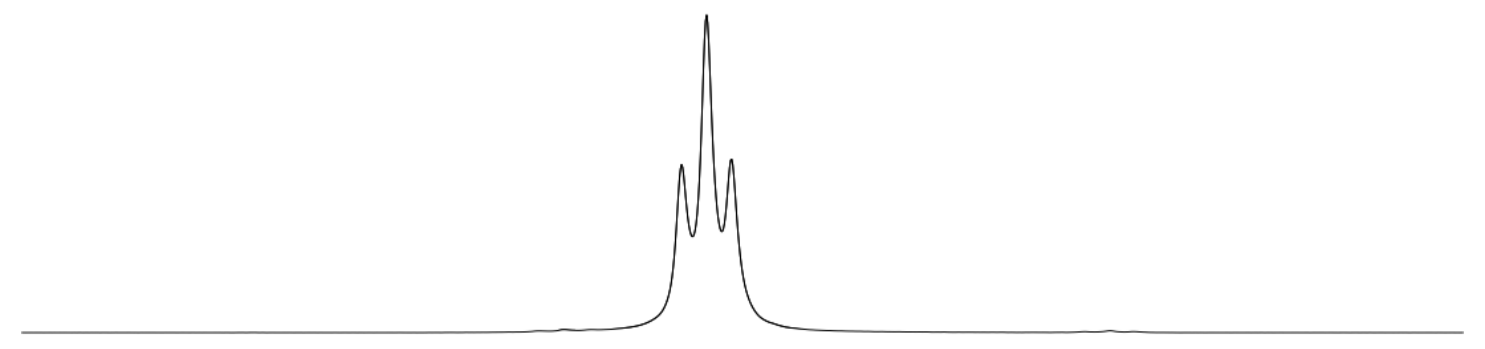

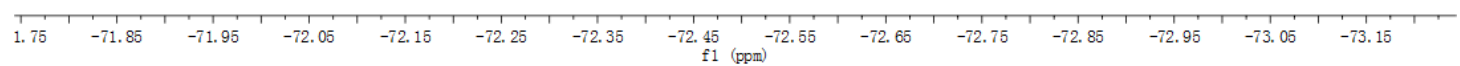

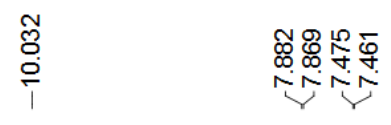

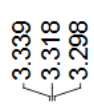

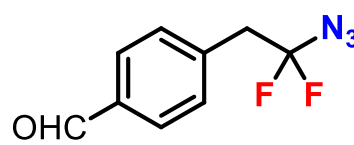

$3 u$

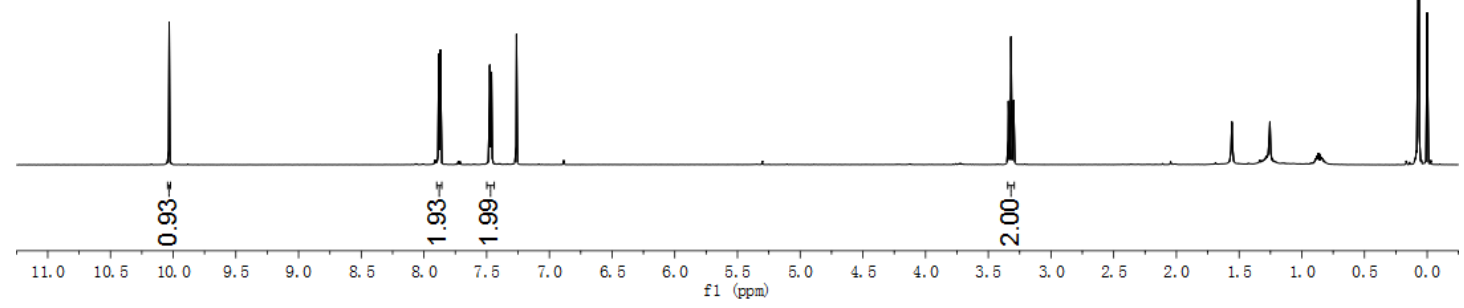



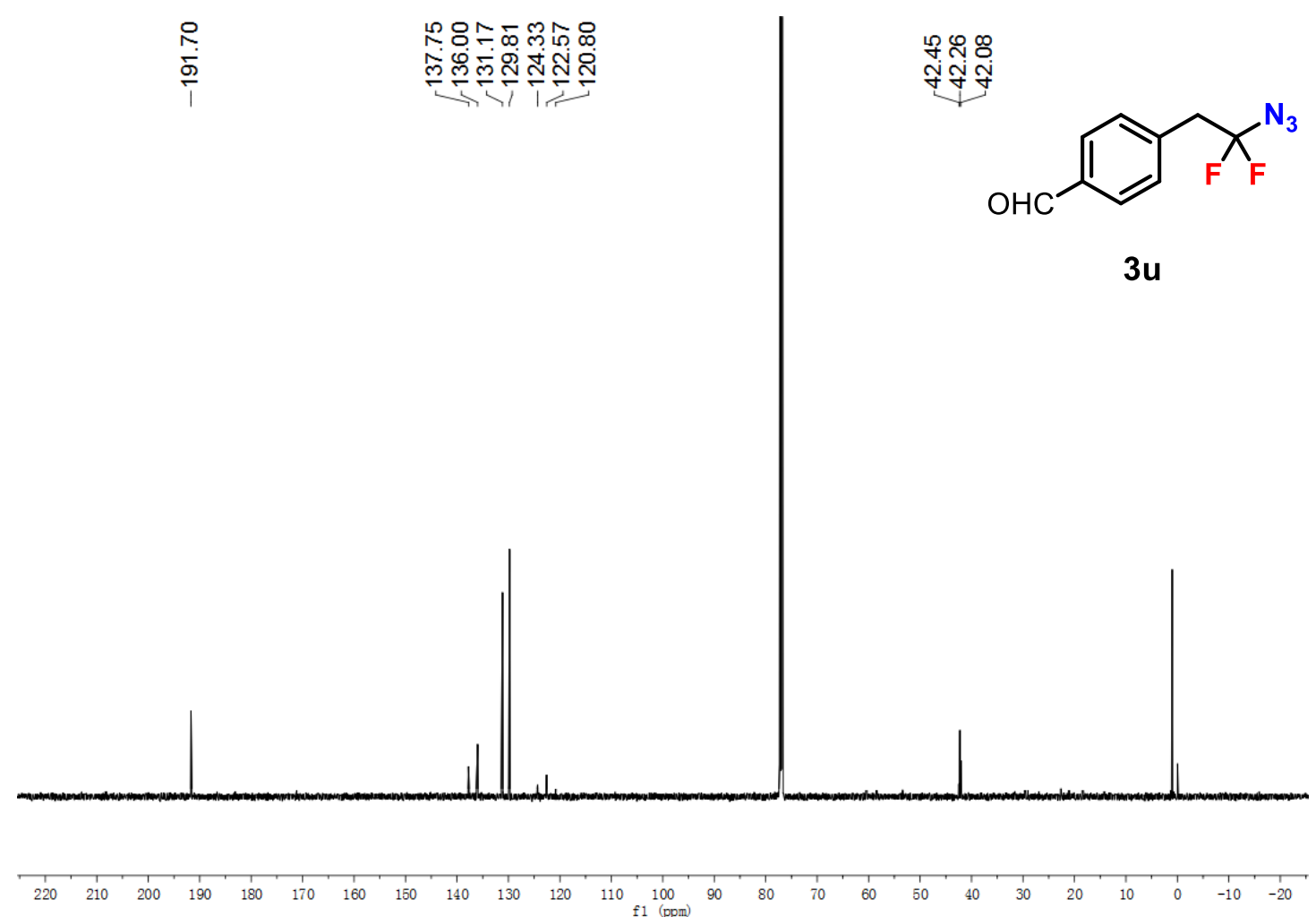

ธูํํำ

잔<smiles>NC(F)(F)Cc1ccc(C=O)cc1</smiles>

$3 u$

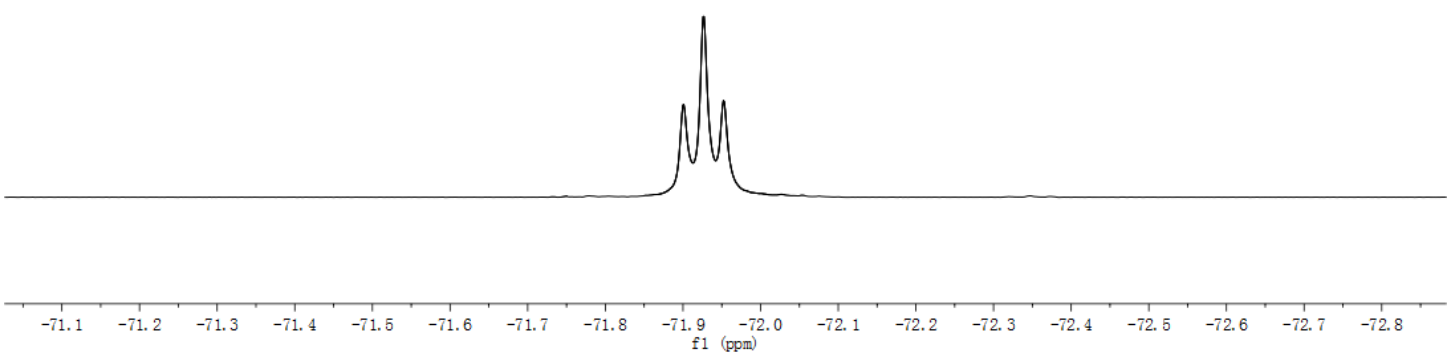




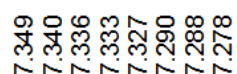

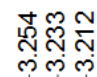

${ }_{3 v}^{N_{3}}$

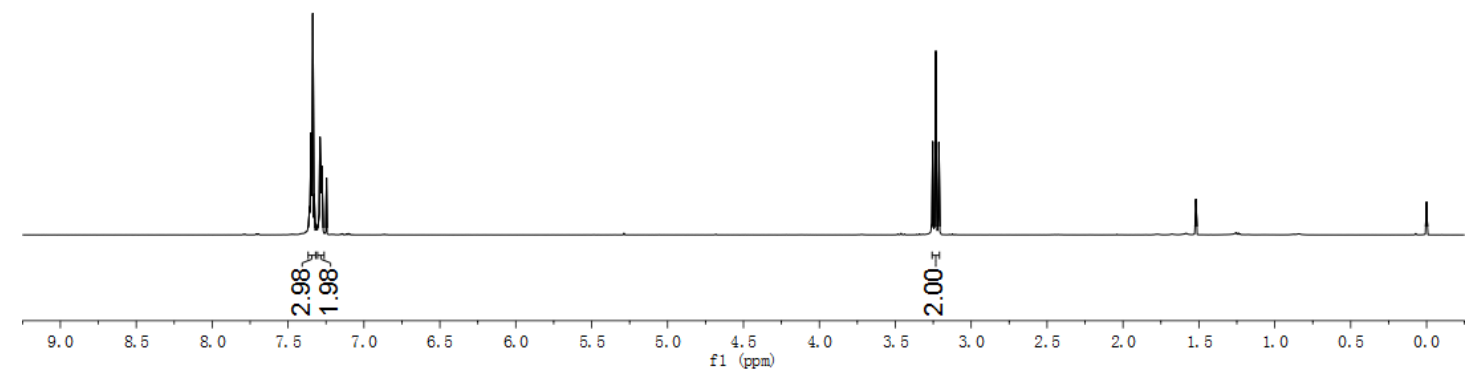

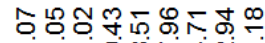

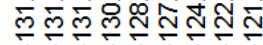

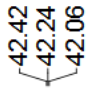
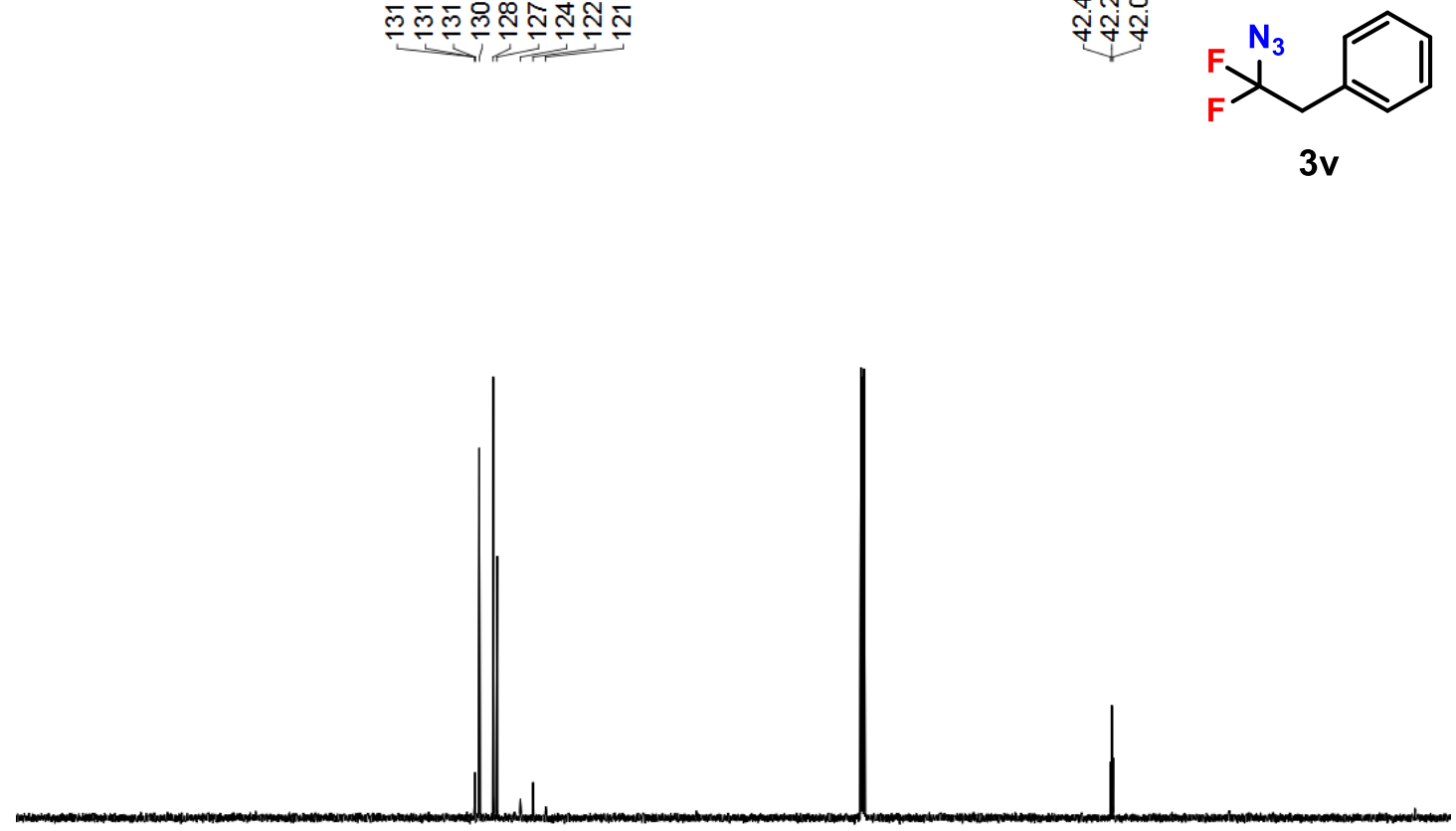

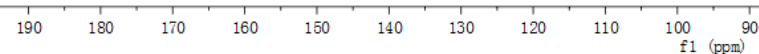


雚

NNㅗㄴ
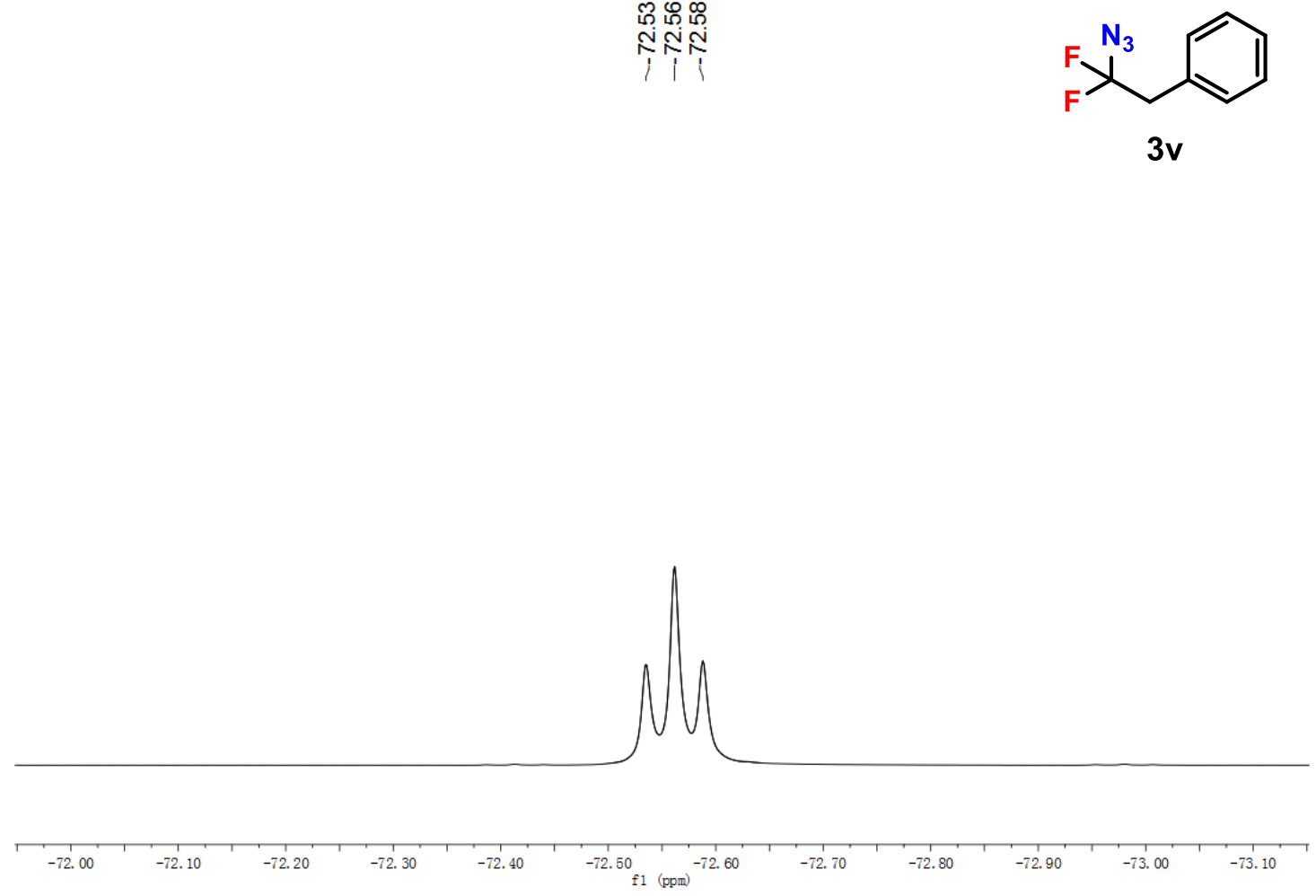

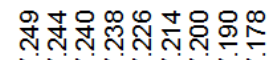

NNNNNNTN

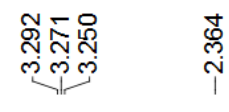
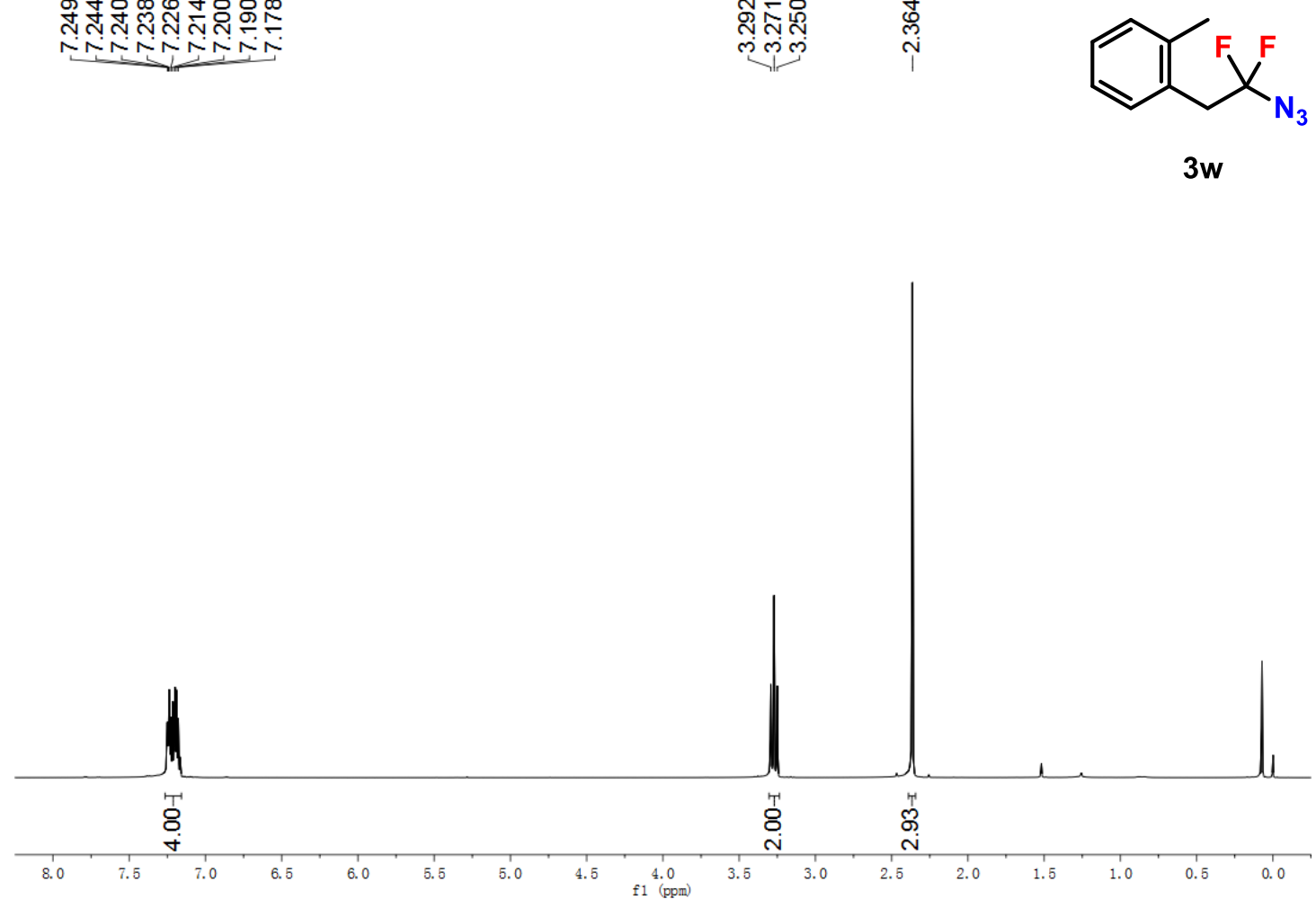


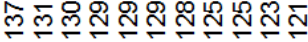

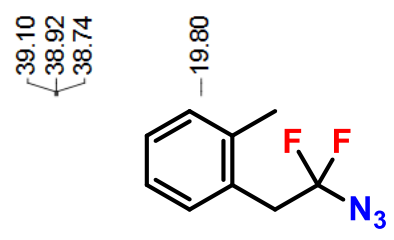

$3 w$
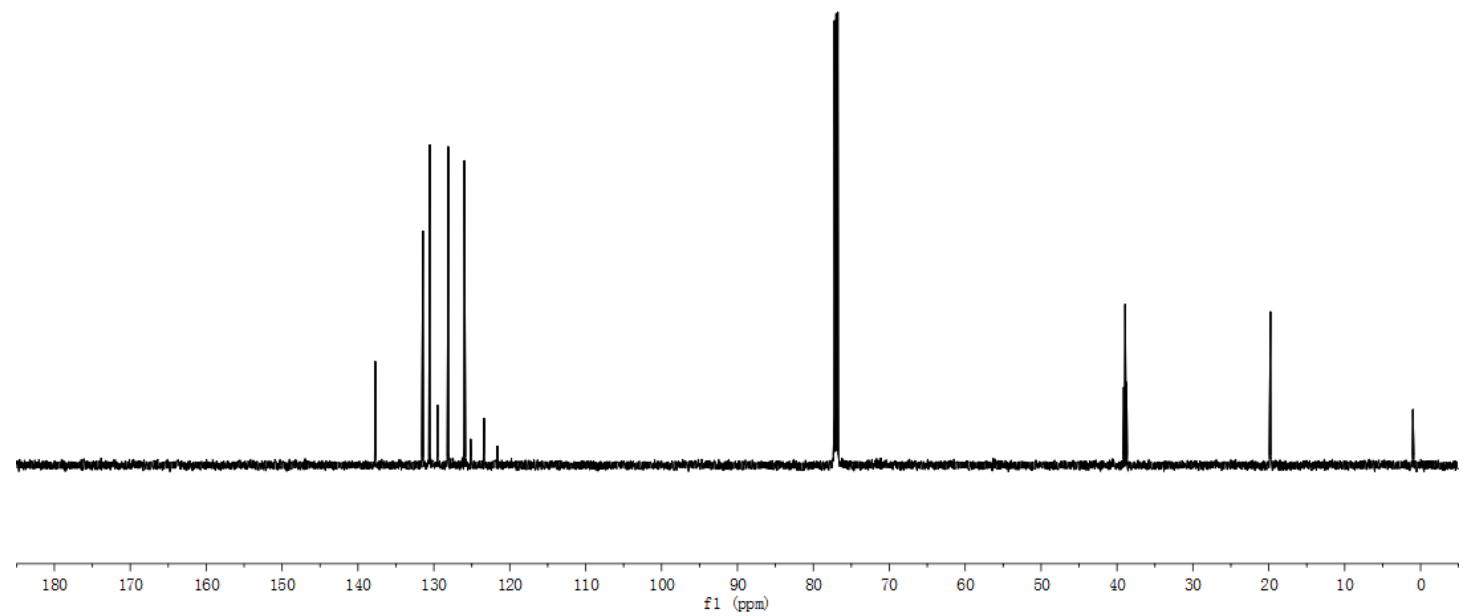

న్

춘

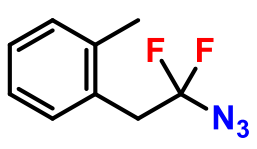

$3 w$

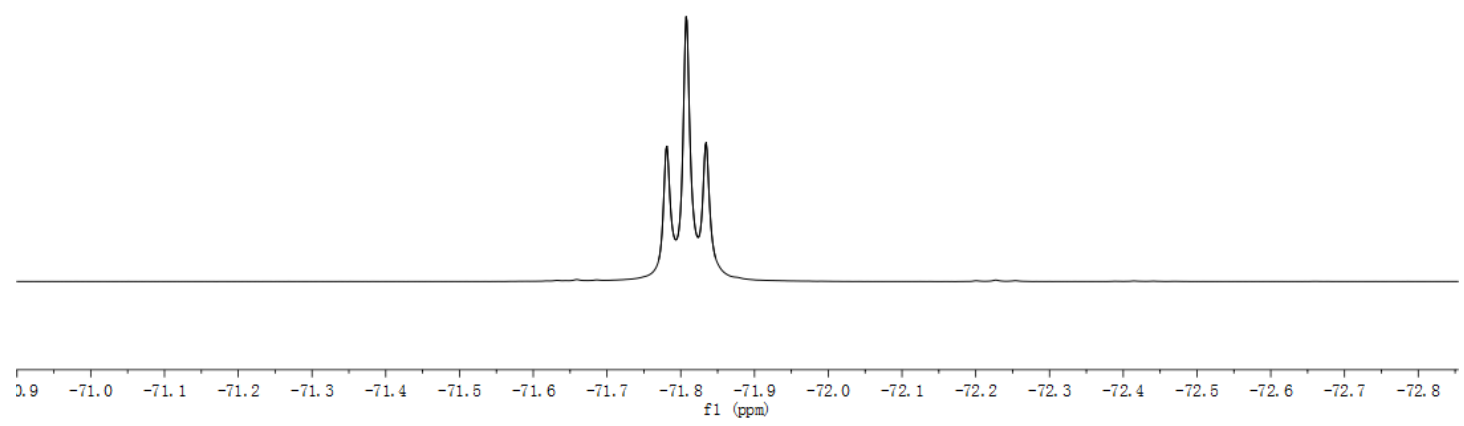




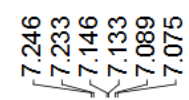

तู

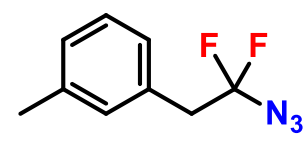

$3 x$

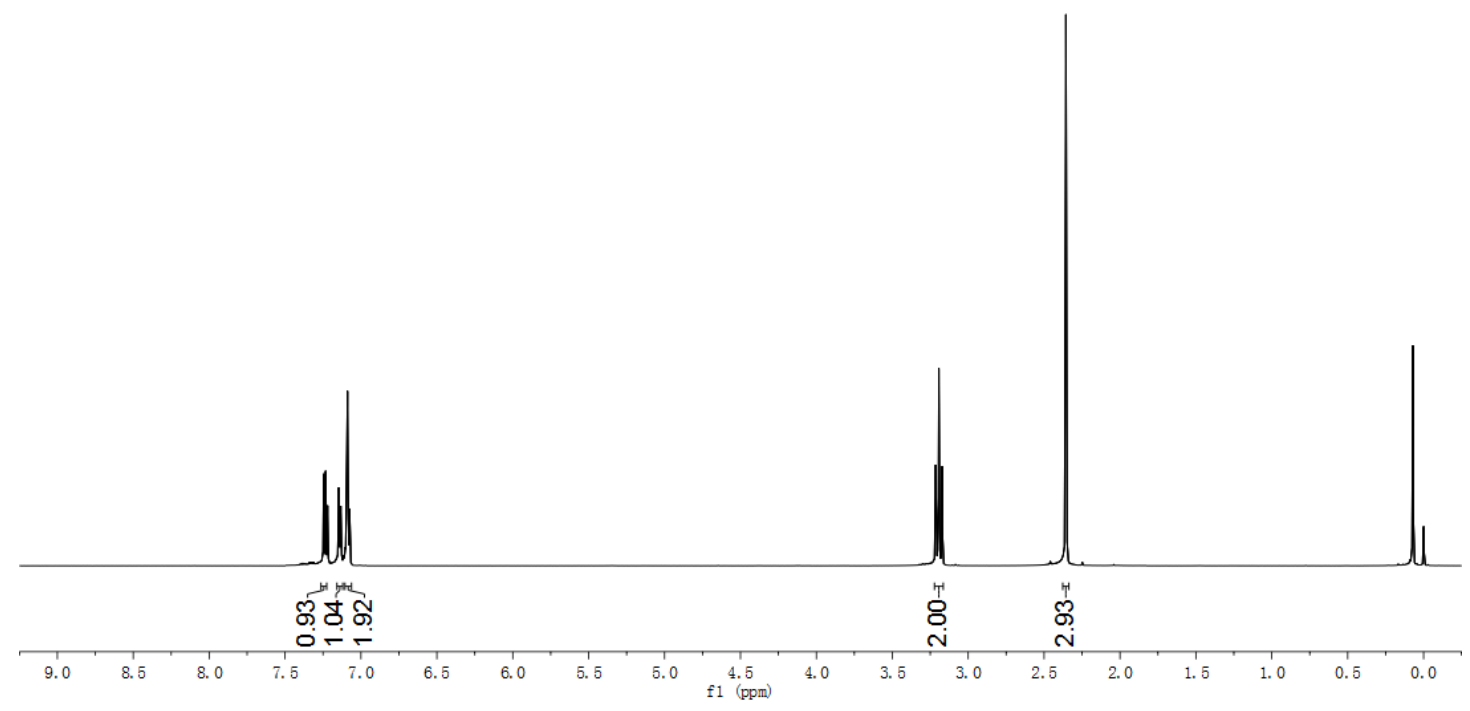

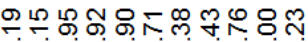

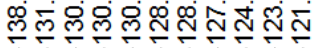

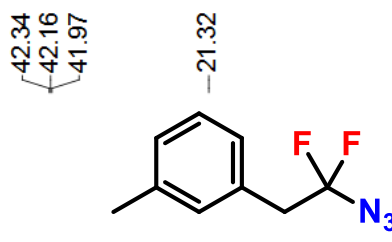

$3 x$

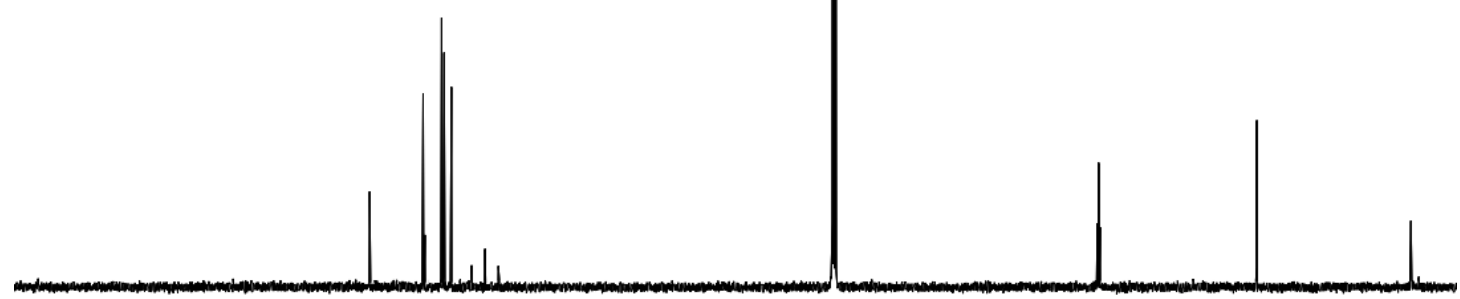

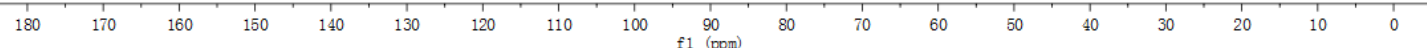




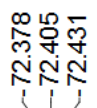

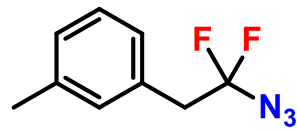

$3 x$

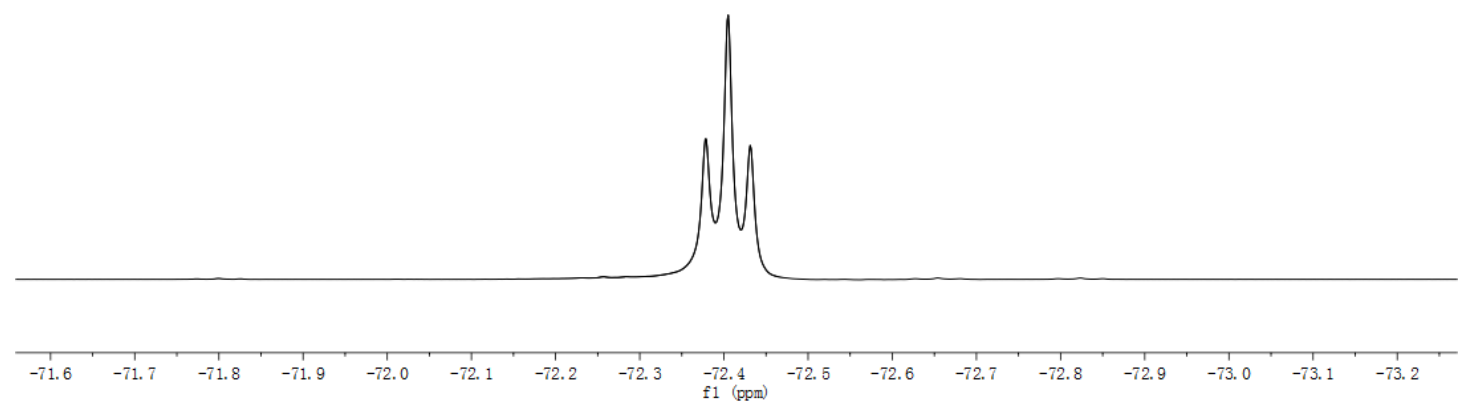

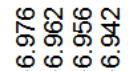

詀骨

>

3y

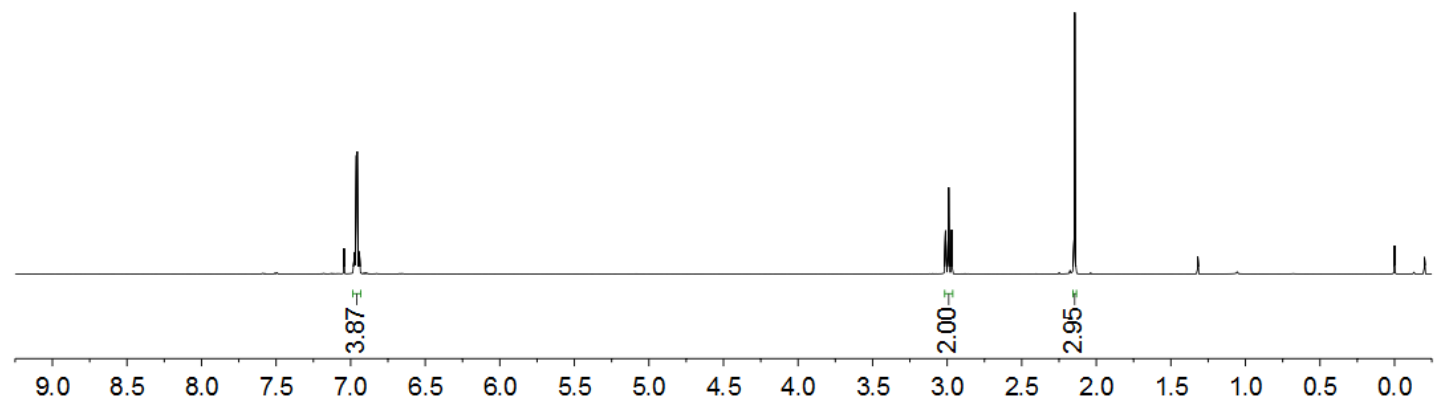


<smiles>Cc1ccc(CC(N)(F)F)cc1</smiles>

3y

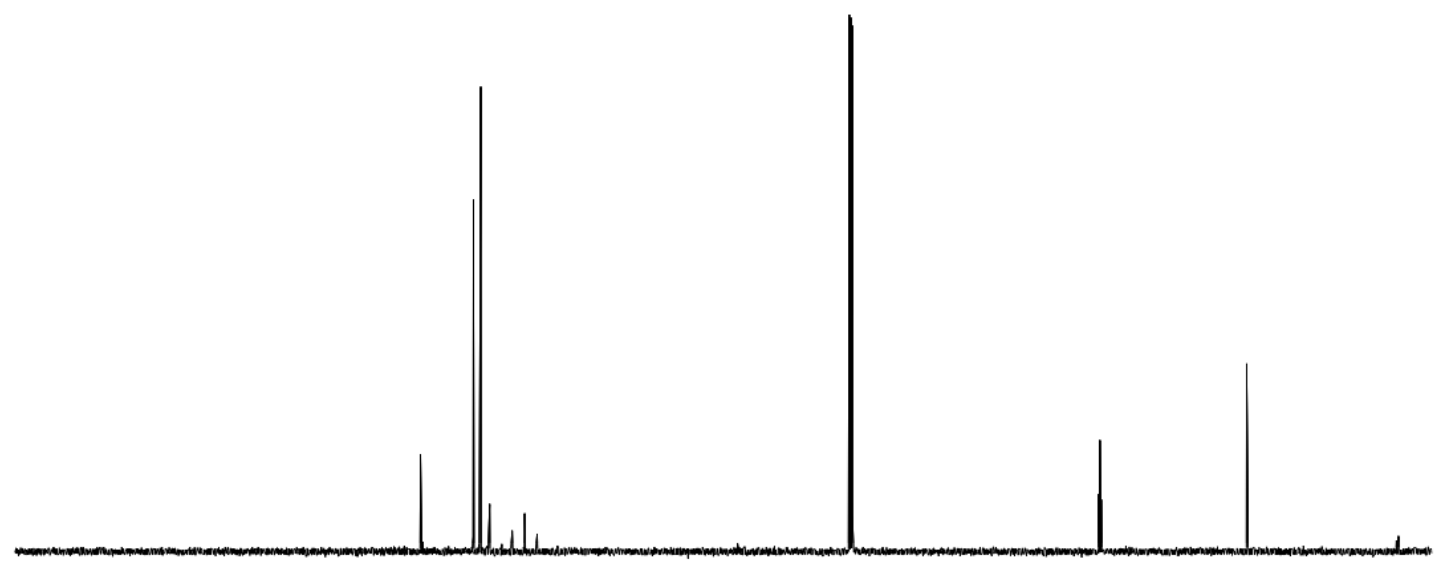

$\begin{array}{llllllllllllllllllll}190 & 180 & 170 & 160 & 150 & 140 & 130 & 120 & 110 & 100 & 90 & 80 & 70 & 60 & 50 & 40 & 30 & 20 & 10 & 0\end{array}$

\section{ஜ:}

논<smiles>Cc1ccc(CC(N)(F)F)cc1</smiles>

3y

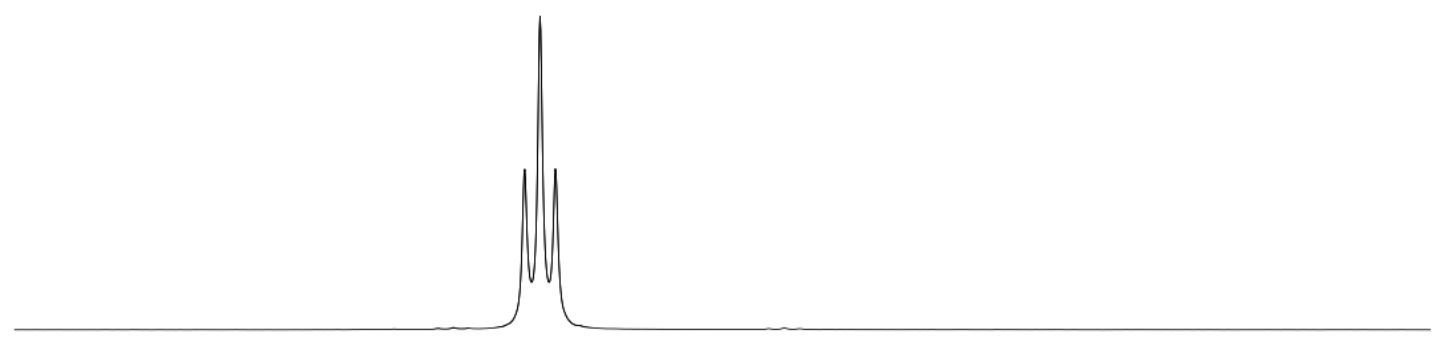

\begin{tabular}{llllllllllll}
\hline-71.8 & -72.0 & -72.2 & -72.4 & -72.6 & -72.8 & -73.0 & -73.2 & -73.4 & -73.6 & -73.8 & -74.0
\end{tabular} 


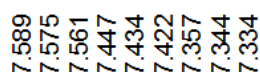

웛웜ำ

लॉल<smiles>NC(F)(F)Cc1ccc(-c2ccccc2)cc1</smiles>

$3 z$
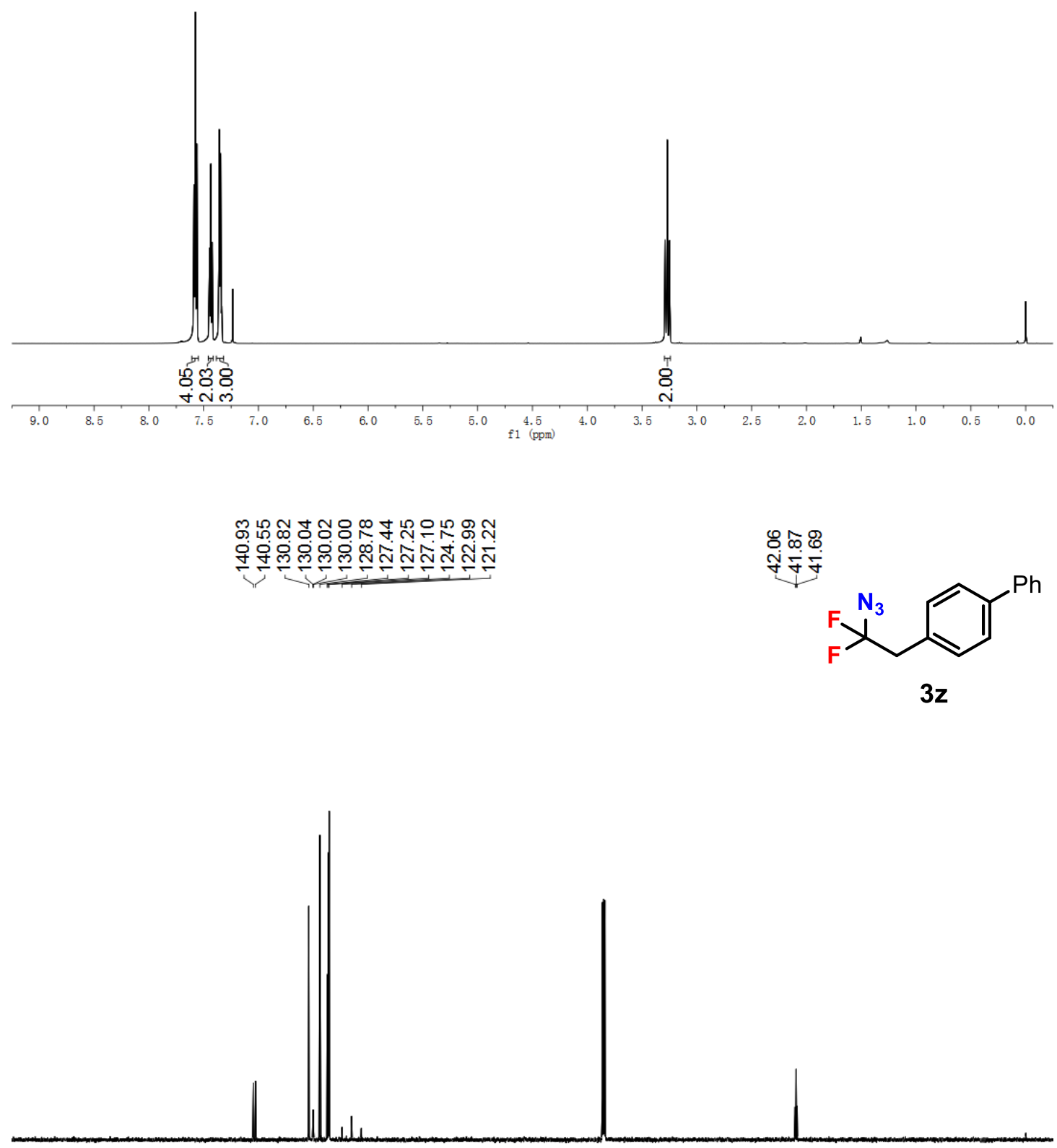

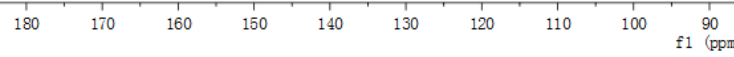




\section{品狳 亭}

กูกำ<smiles>NC(F)(F)Cc1ccc(-c2ccccc2)cc1</smiles>

$3 z$

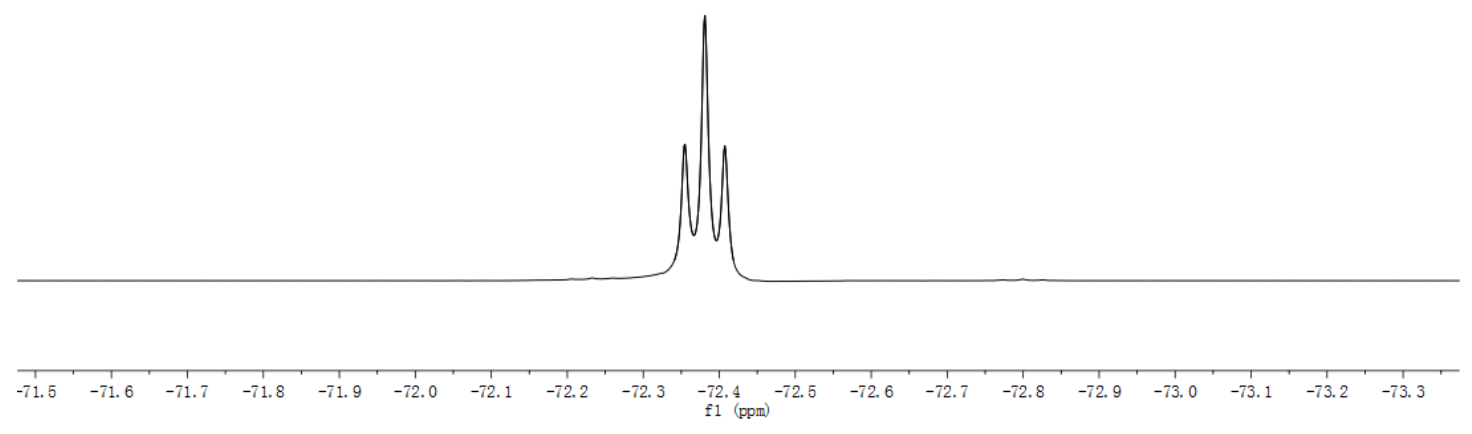

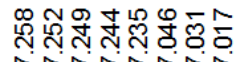

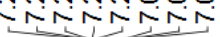

స్ํํำ
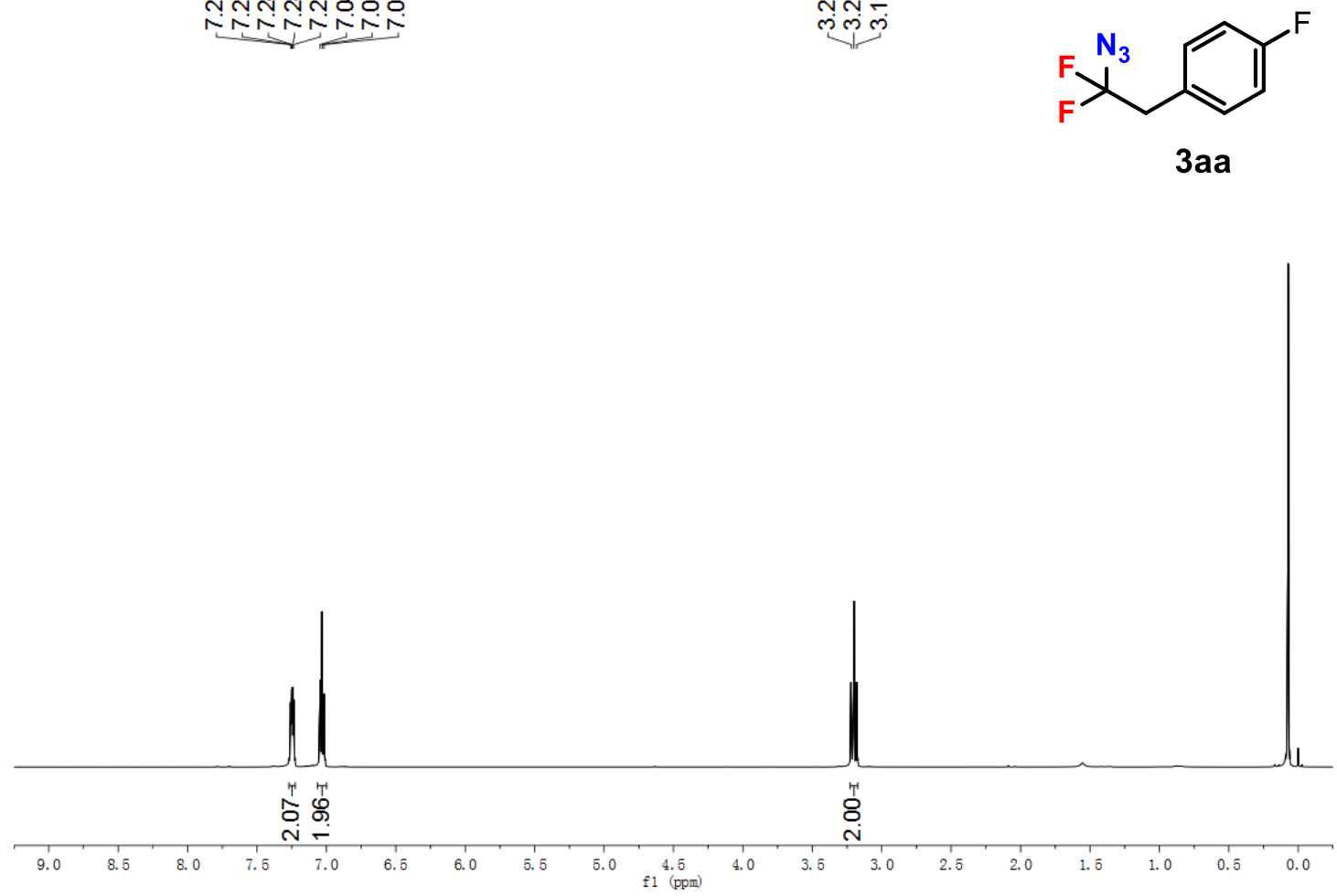


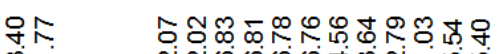

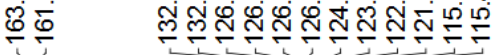

৫ํำ

过字

${ }^{\mathrm{N}_{3}}$

3aa
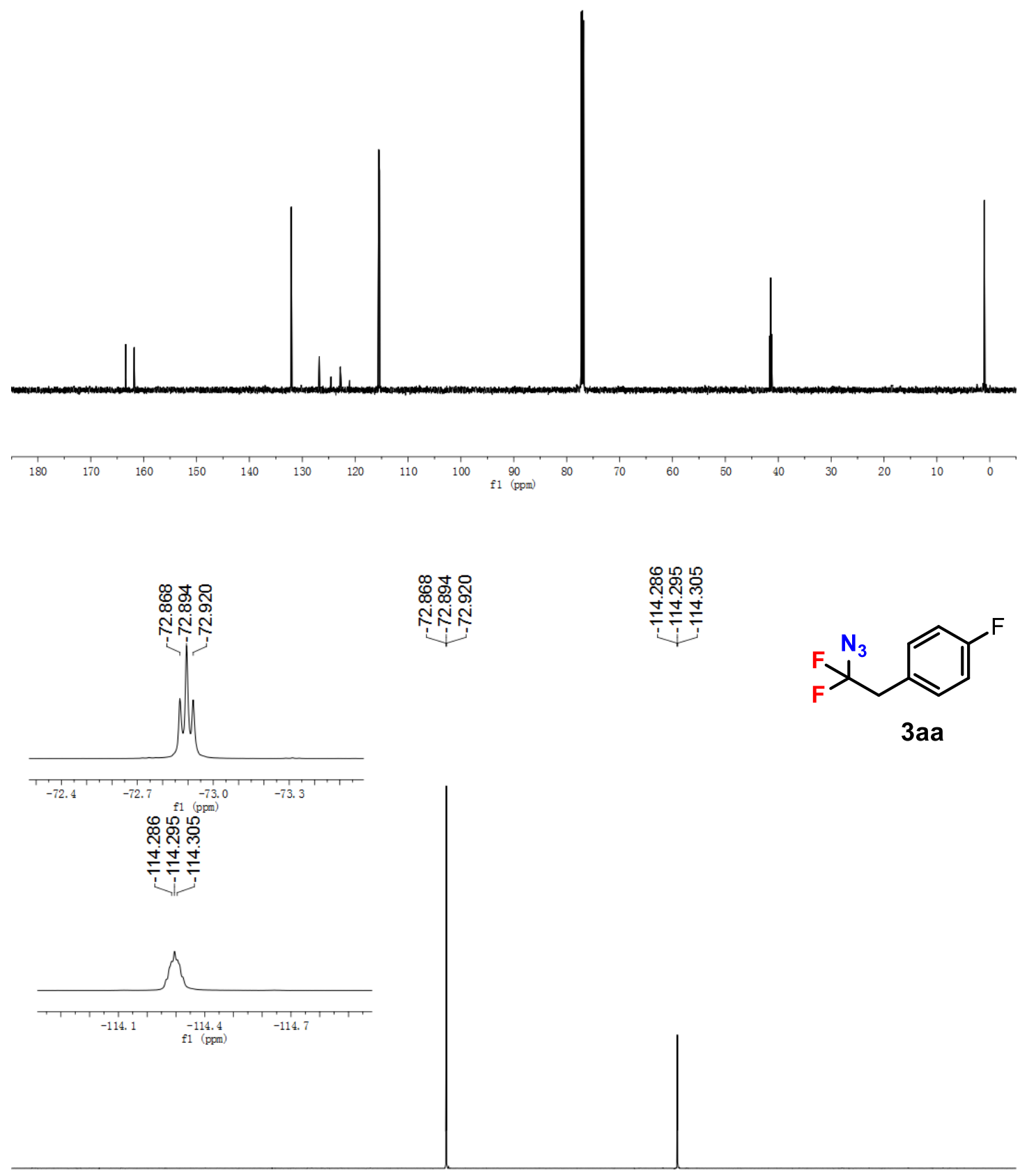

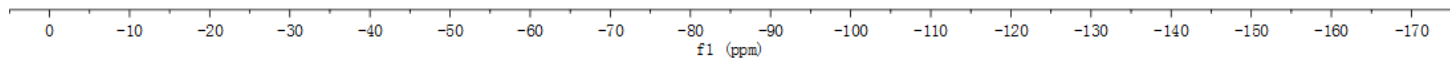




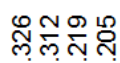

Nín

तั लำ

${ }^{\mathrm{N}_{3}}$

$3 a b$

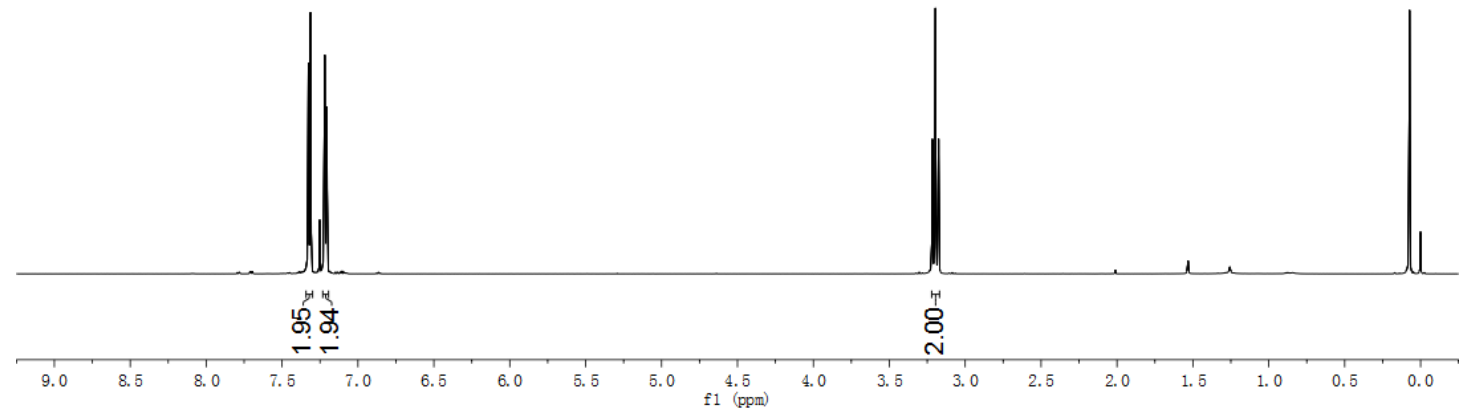

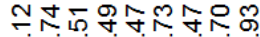

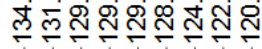

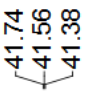
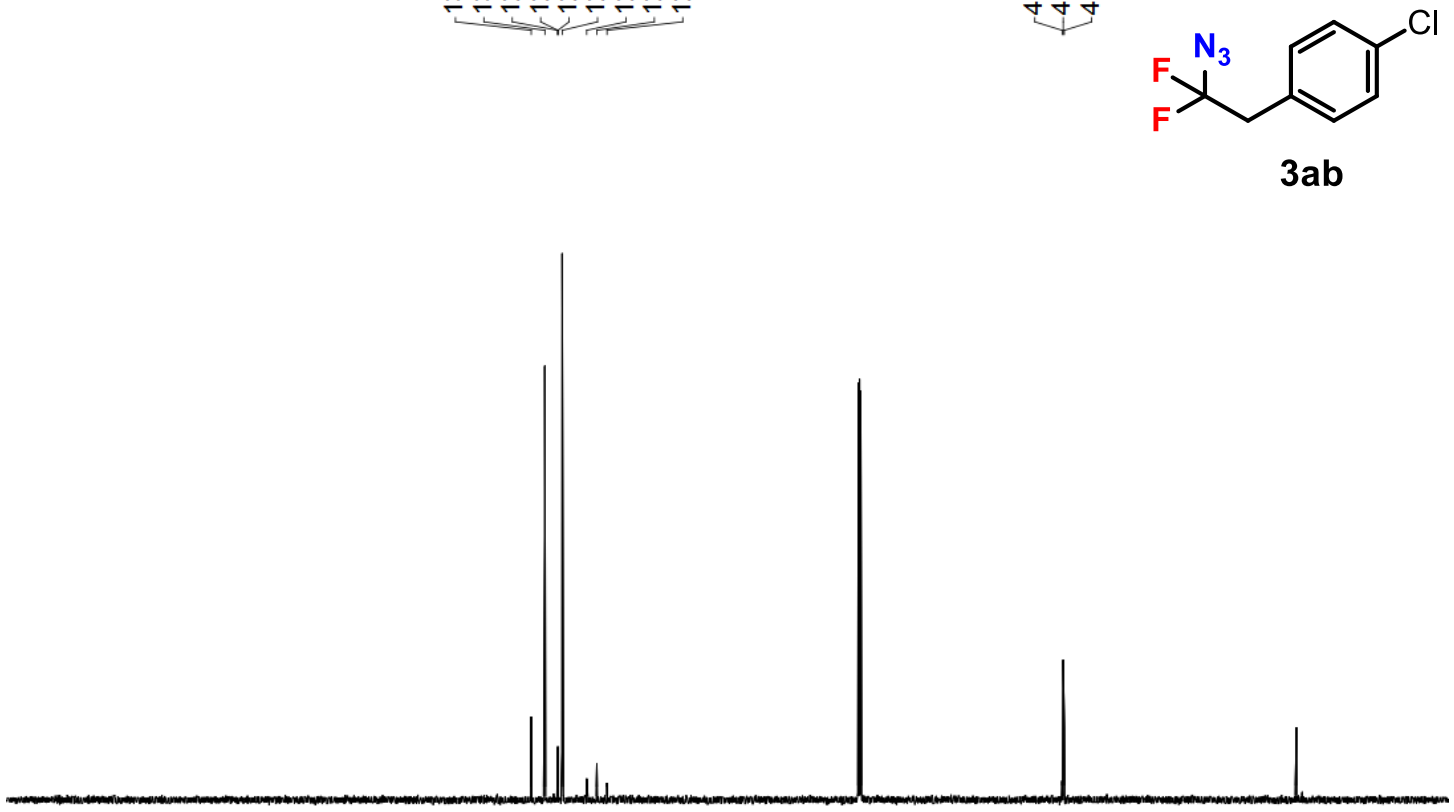

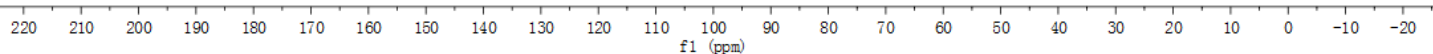


ח
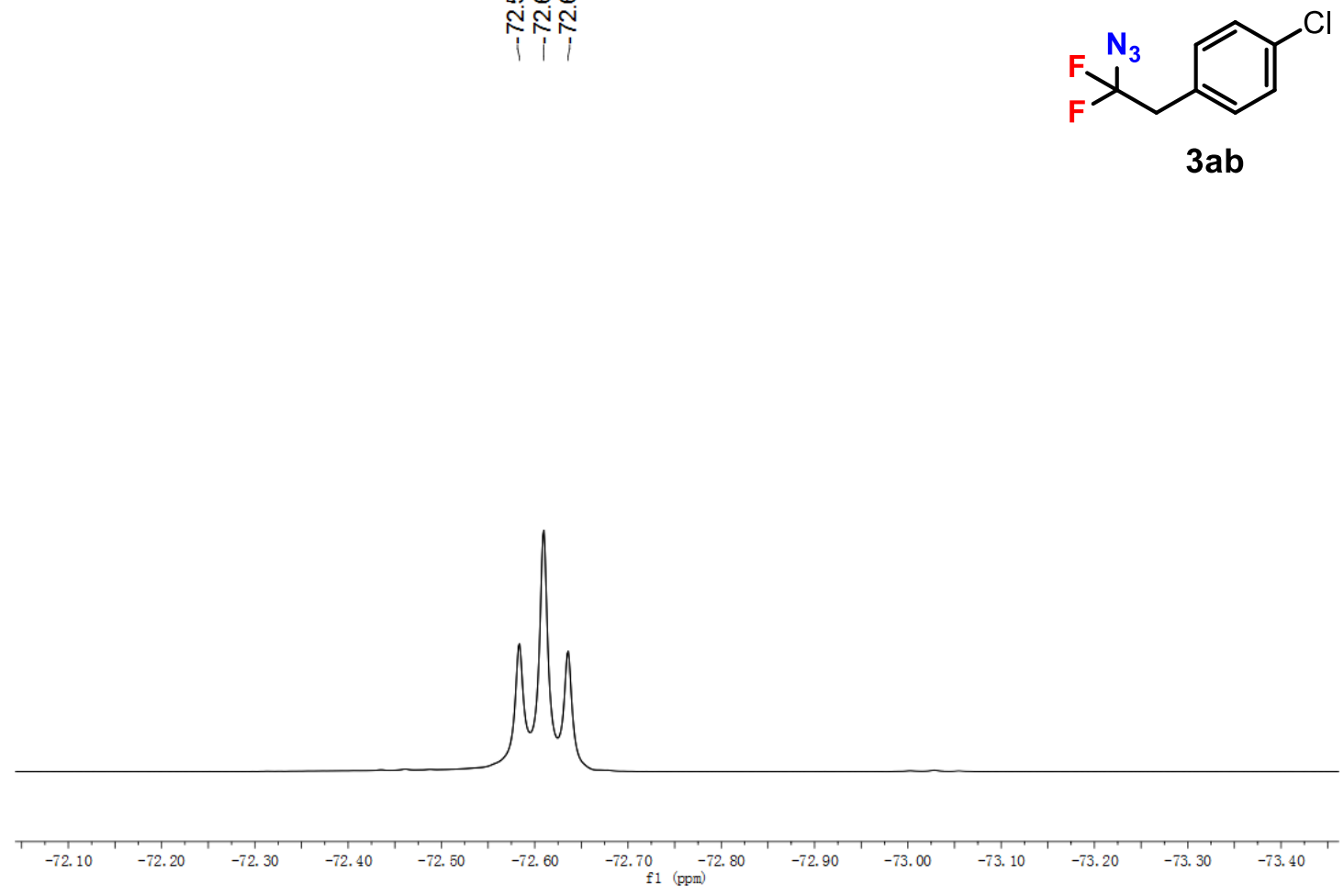

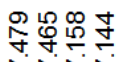

NN

迎

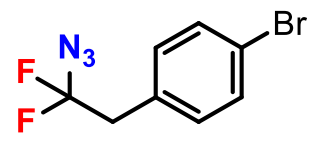

3ac

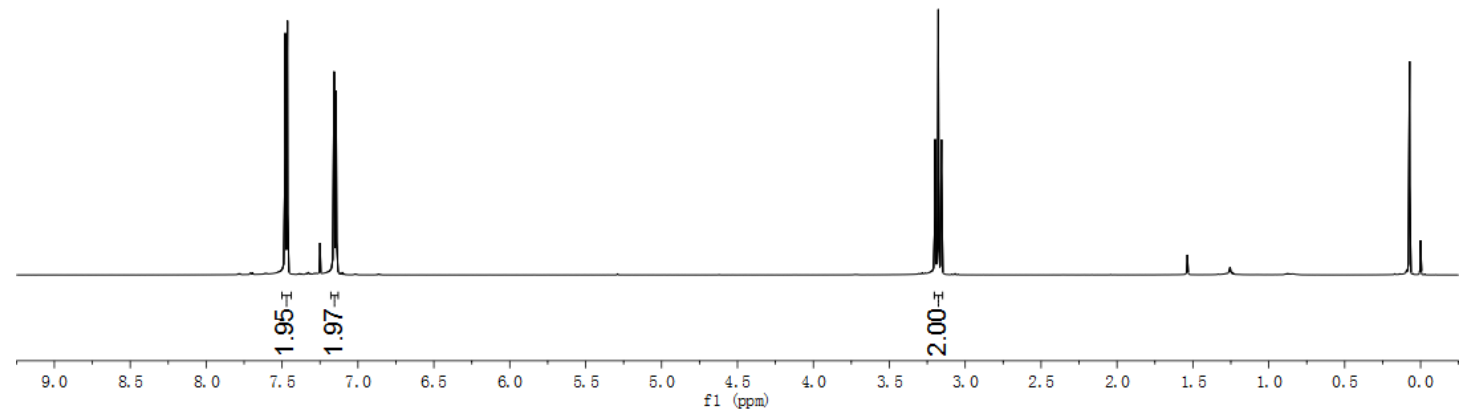



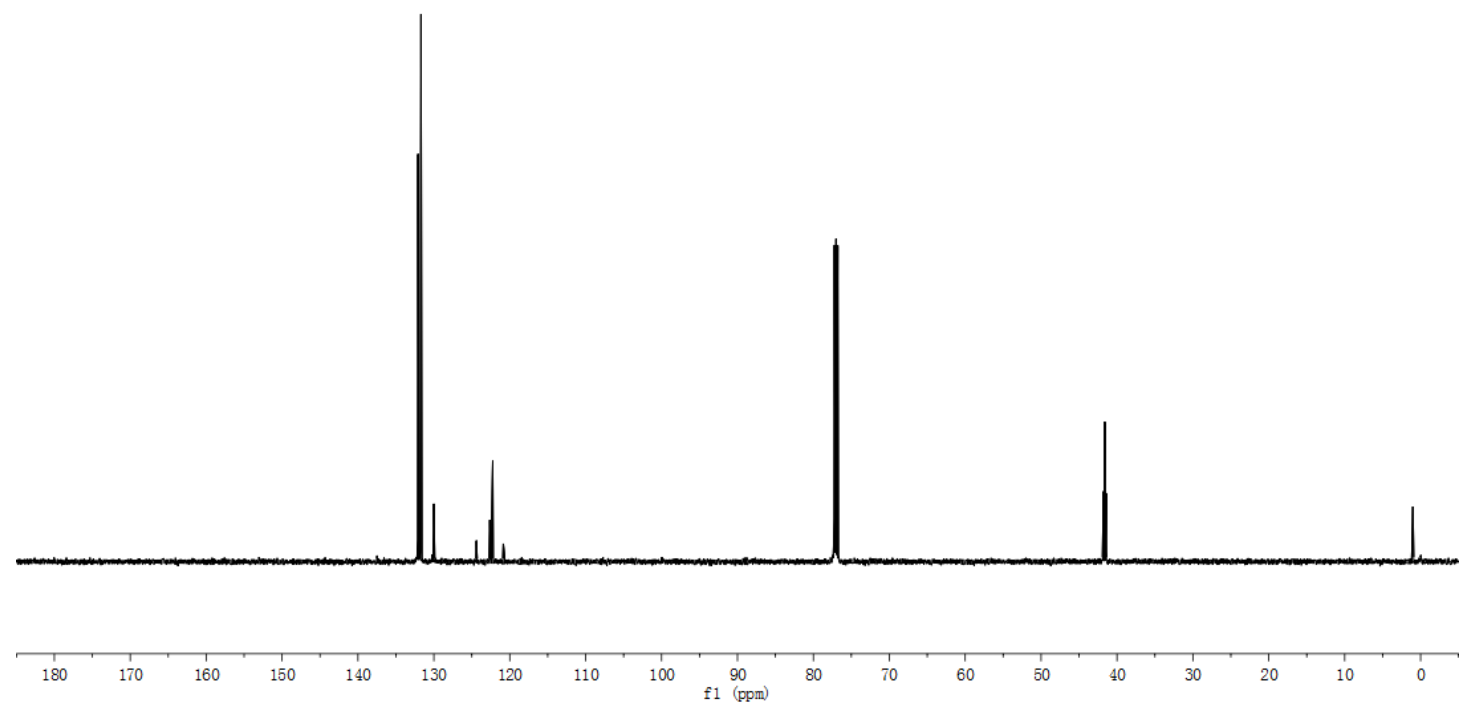

유요유.

춧ํㄴ<smiles>NC(F)(F)Cc1ccc(Br)cc1</smiles>

3ac

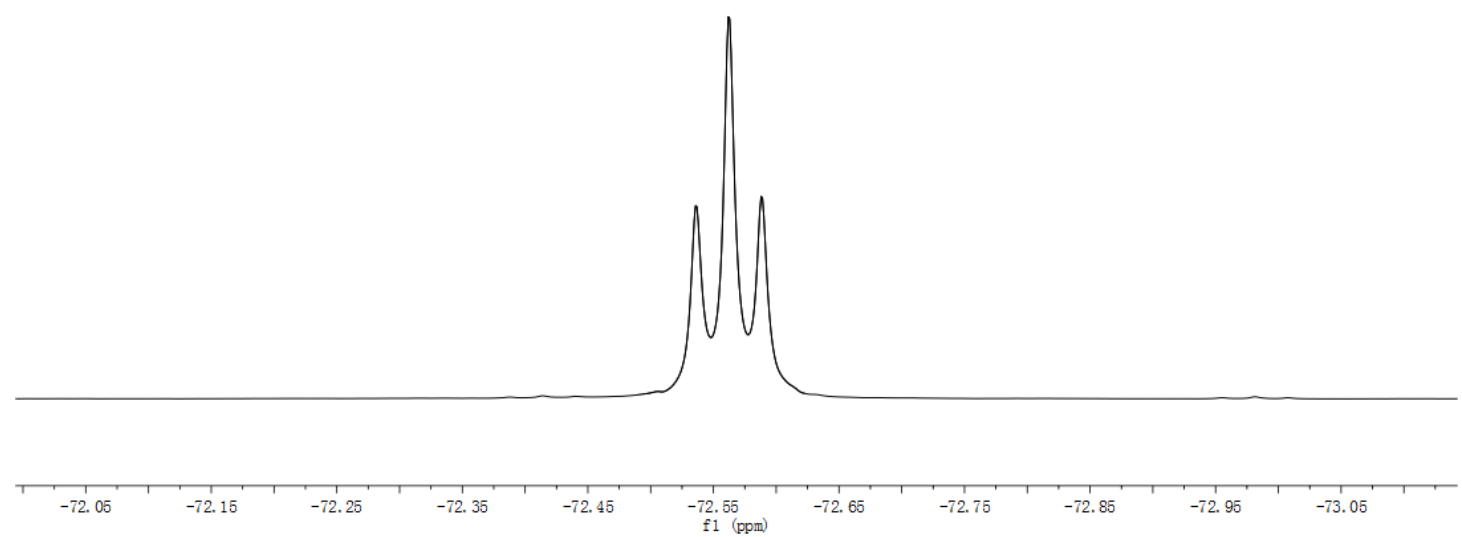




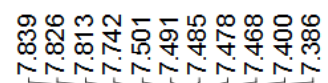

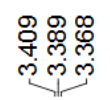

$\overbrace{3 a d} x_{F}^{N_{3}}$
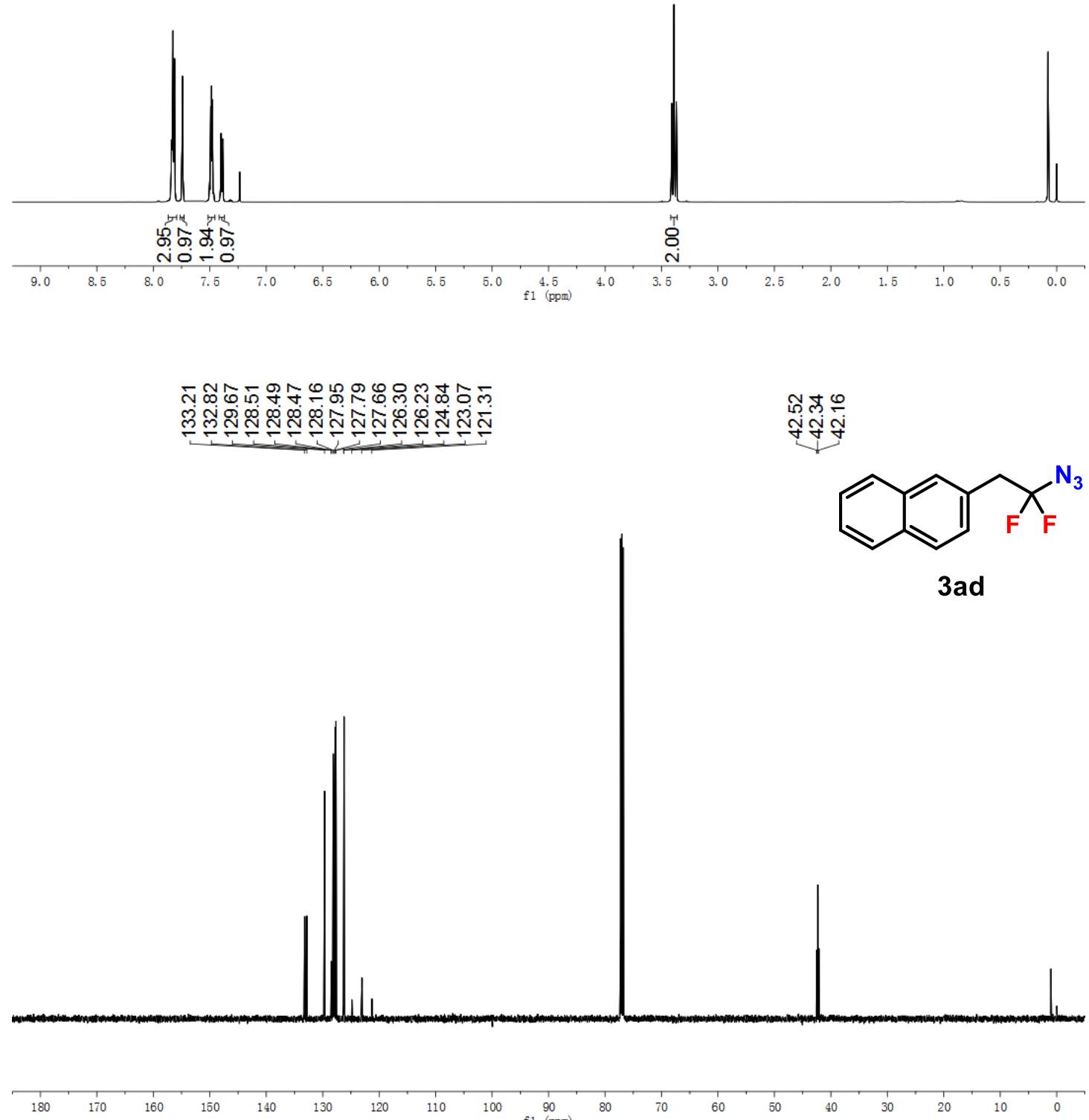

$\begin{array}{lll}100 & 90 \\ \mathrm{f} 1 & 90 \\ (\mathrm{ppm})\end{array}$ 

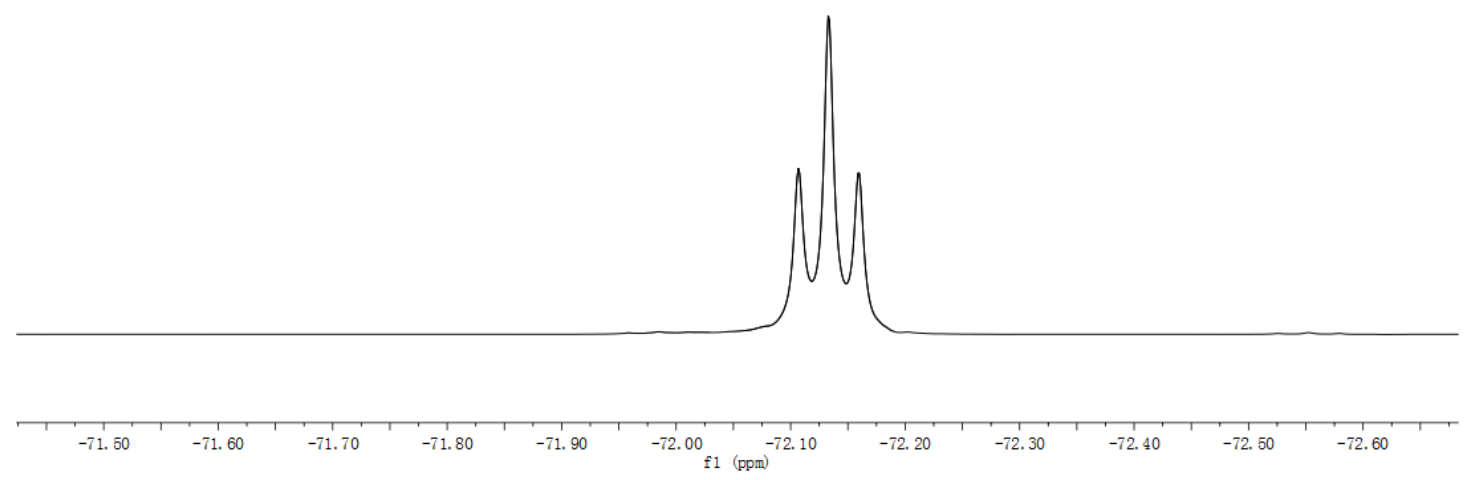

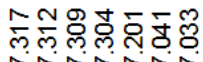

NNNNN

్ㅜㄹํำ

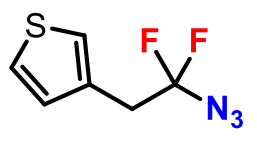

$3 a e$

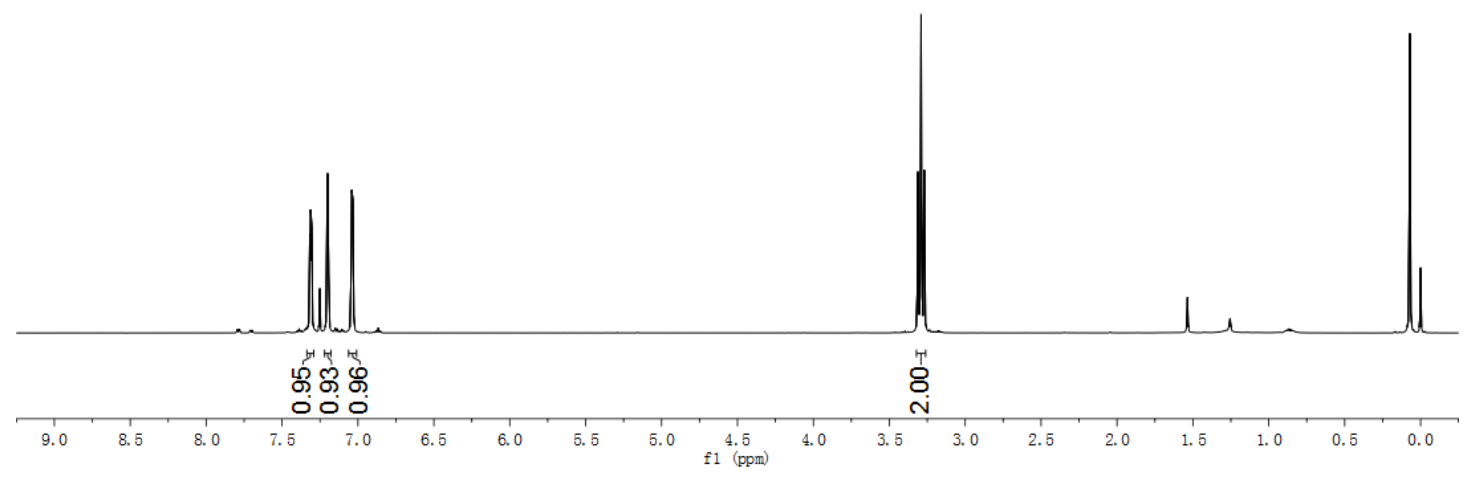



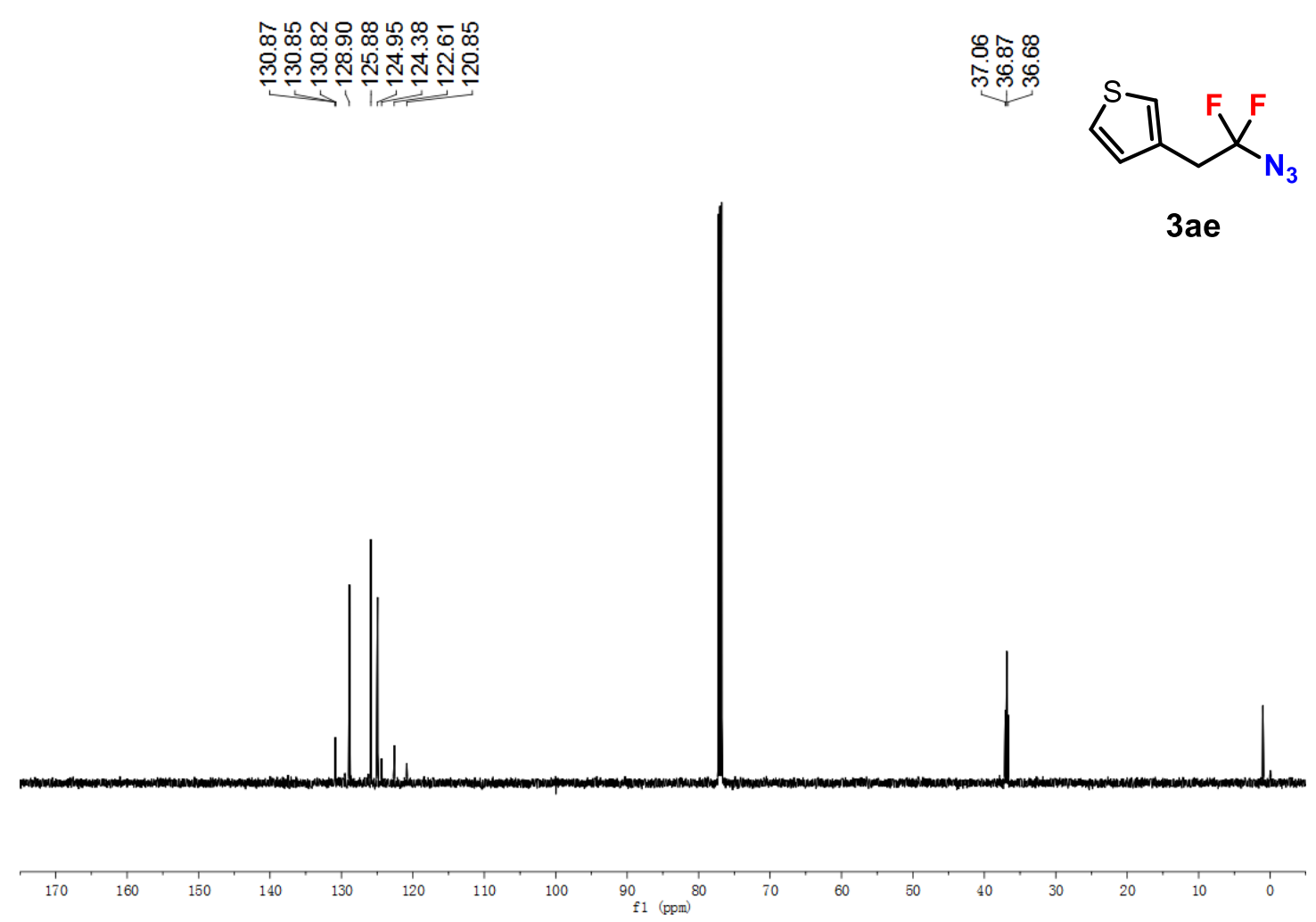

的余兽

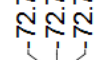

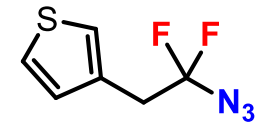

$3 a e$

$\begin{array}{rrrrrrrrrrrrrrrrrrrrrrrrrrrr}71.8 & -71.9 & -72.0 & -72.1 & -72.2 & -72.3 & -72.4 & -72.5 & -72.6 & -72.7 & -72.8 & -72.9 & -73.0 & -73.1 & -73.2 & -73.3 & -73.4 & -73.5 & -73.6 & -73.7 & -73.8 & -73.9\end{array}$ 


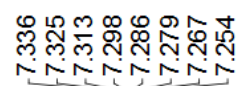

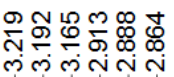
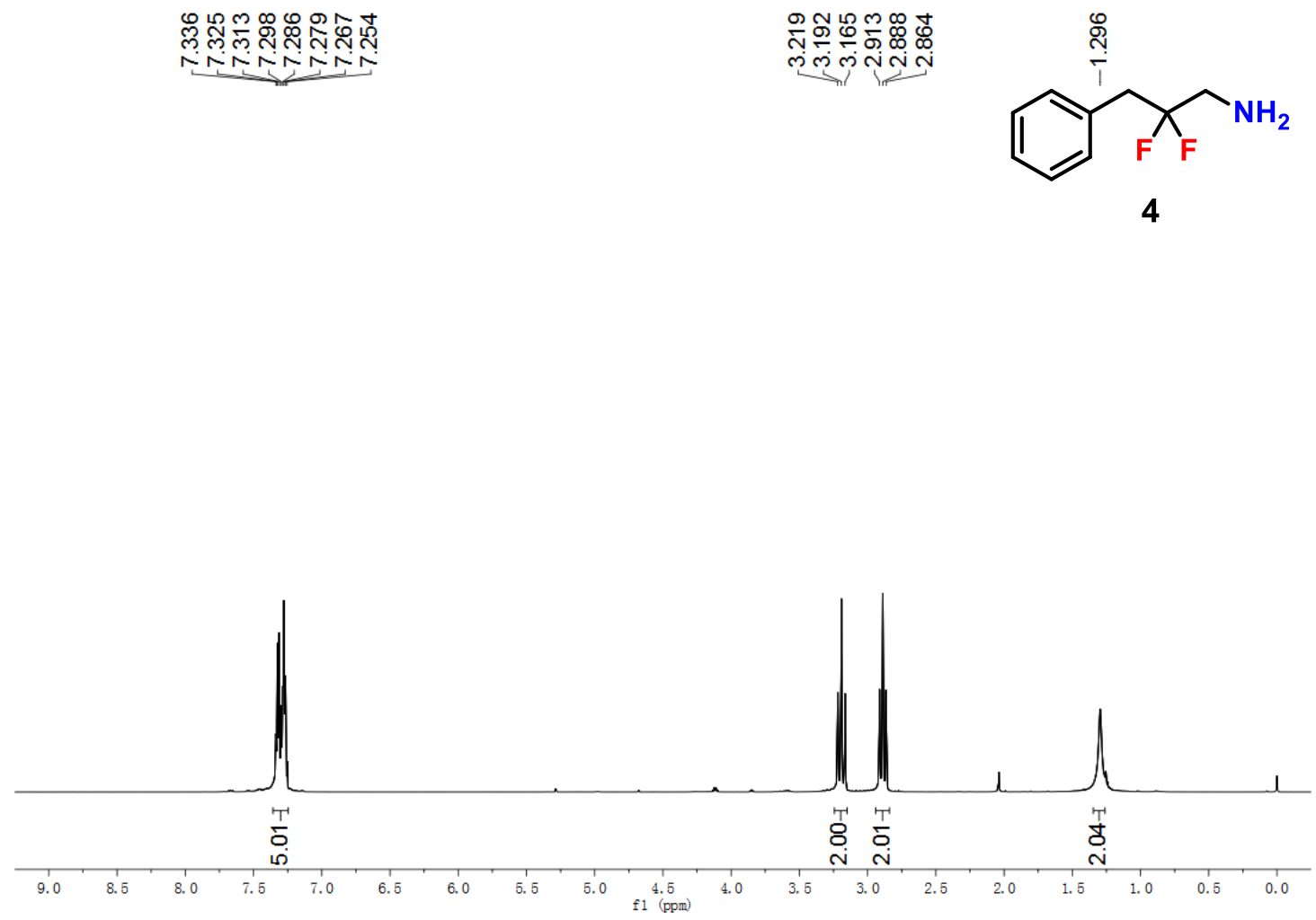

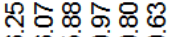

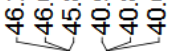<smiles>NCC(F)(F)Cc1ccccc1</smiles>

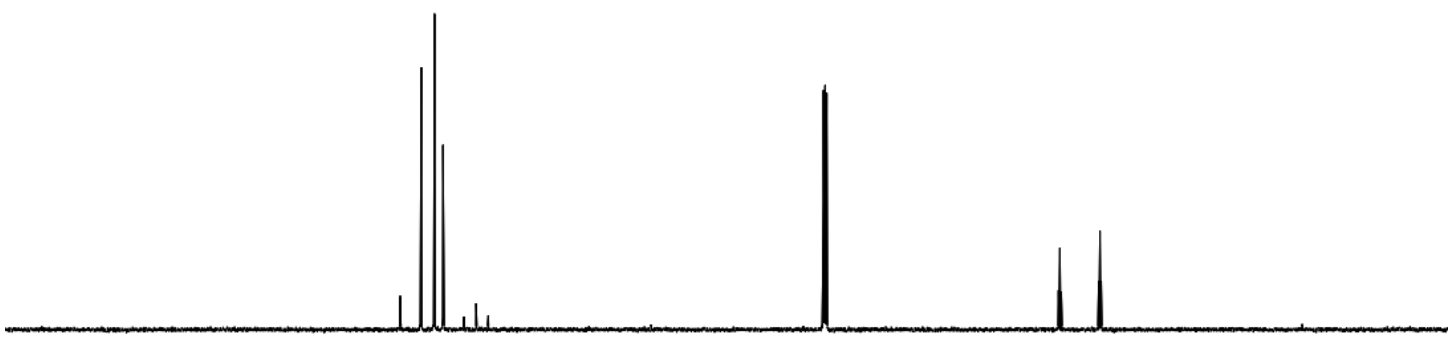

180

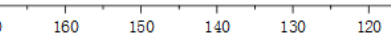

$\begin{array}{lll}100 & 90 \\ \mathrm{f} 1 & 90 \\ (\mathrm{ppm})\end{array}$ 

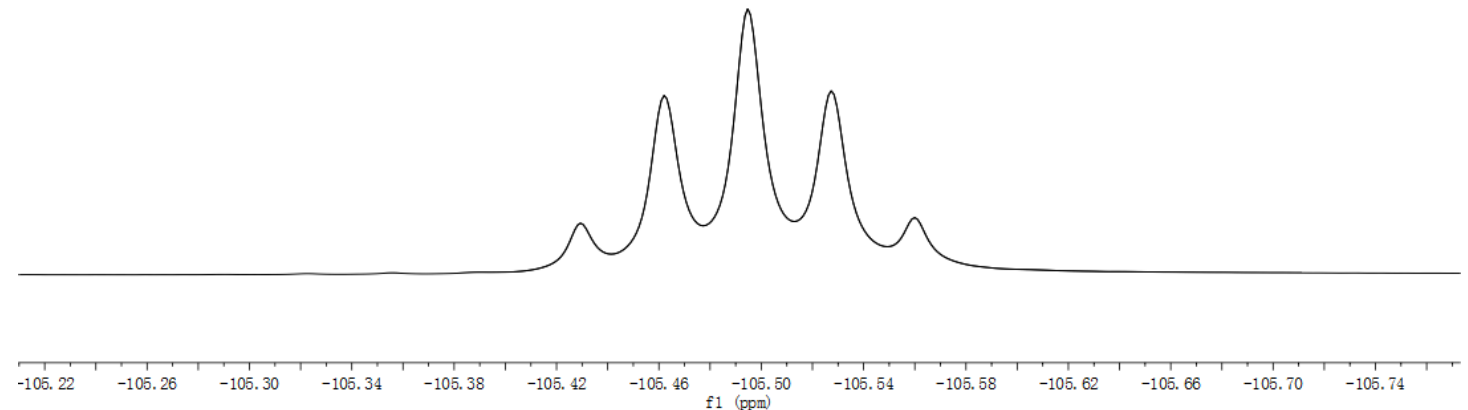

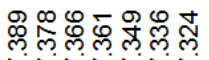

NNNNo

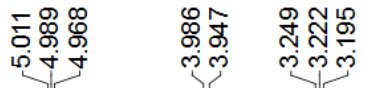

$\overbrace{\mathrm{F}}^{\mathrm{F}_{\mathrm{F}}} \underbrace{\mathrm{N}}_{\mathrm{N}=\mathrm{N}} \mathrm{COOCH}_{3}$

5

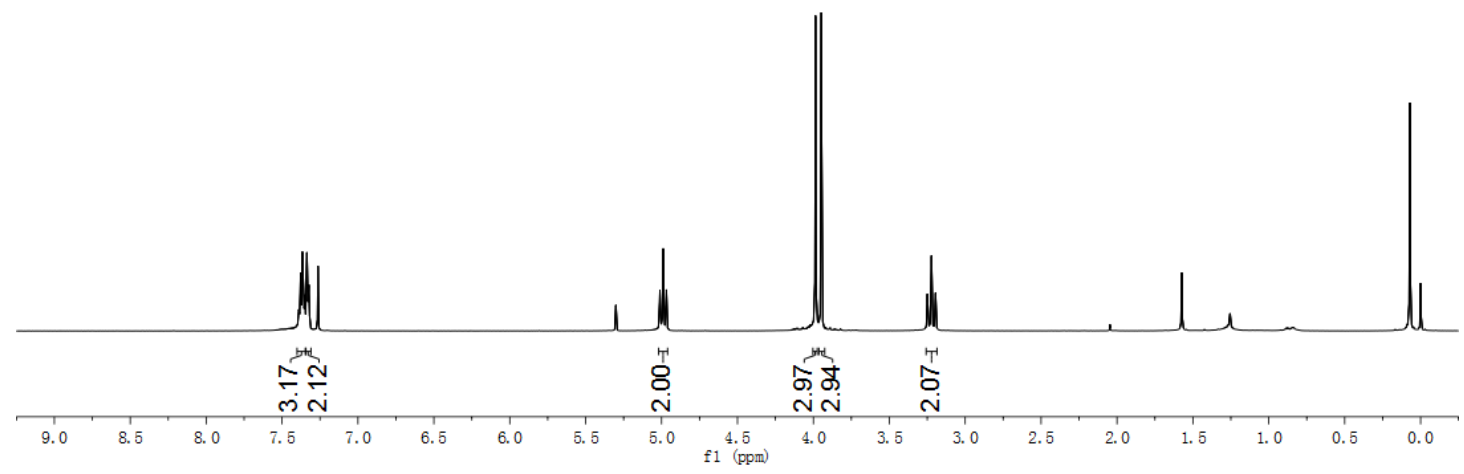



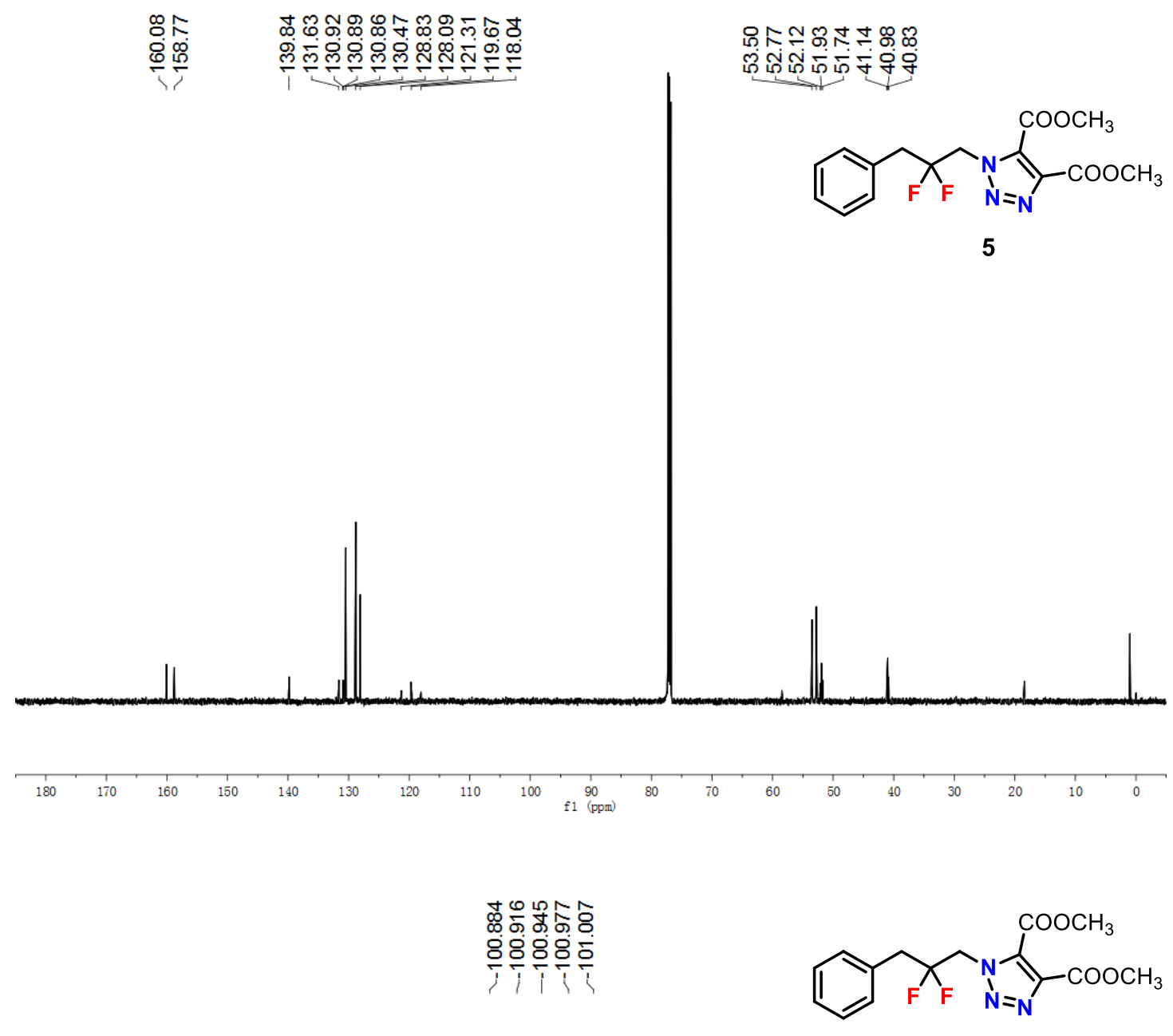

5

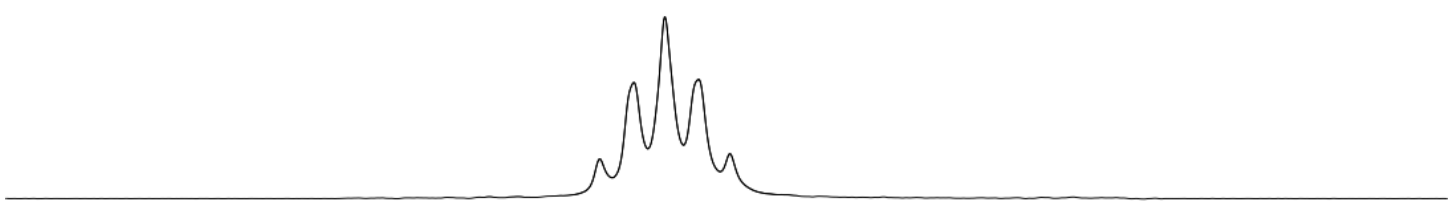




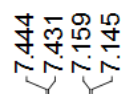

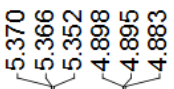

ণ্ণ<smiles>[2H]/C(N)=C(\N)c1ccc(C)cc1</smiles>

$\left[D_{2}\right]^{-6}$

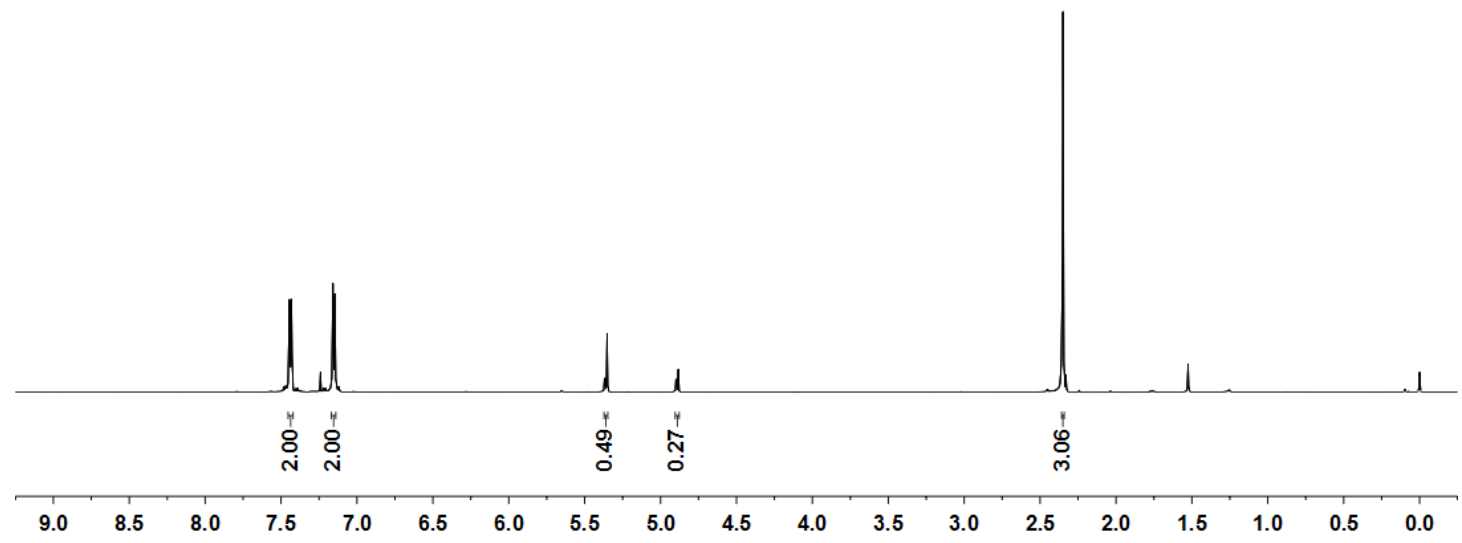

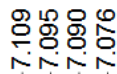

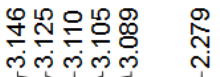
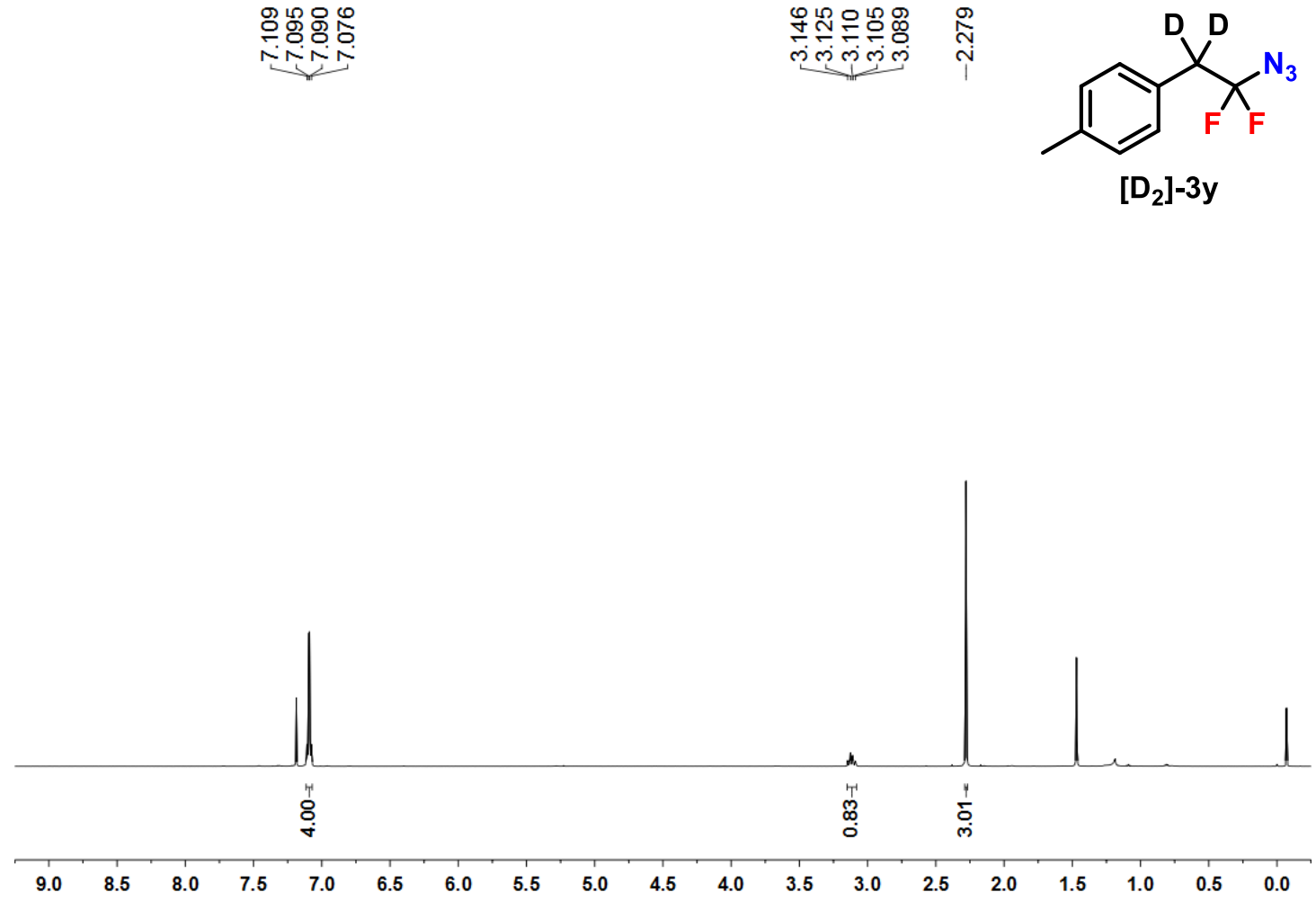

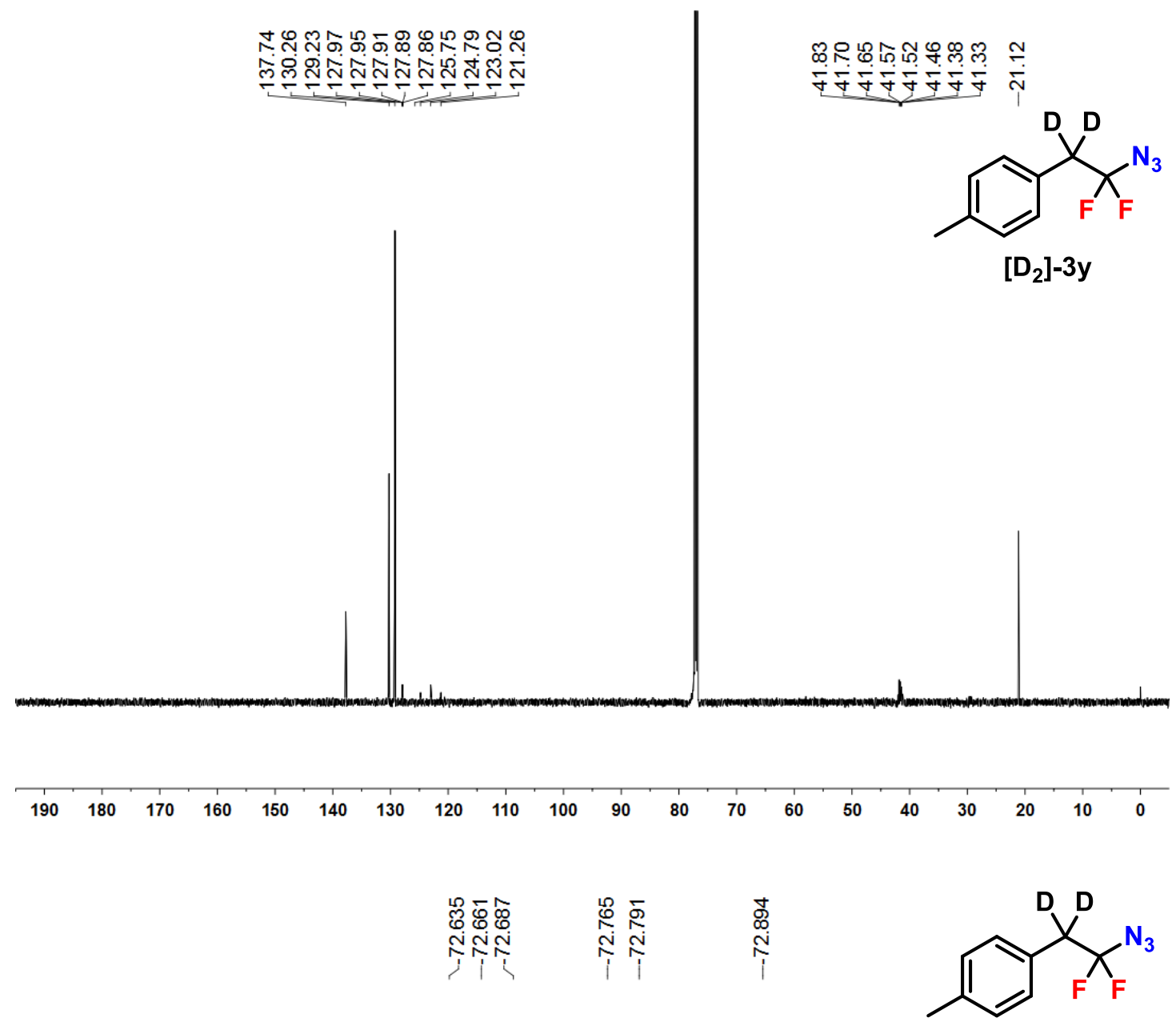

$\left[D_{2}\right]-3 y$

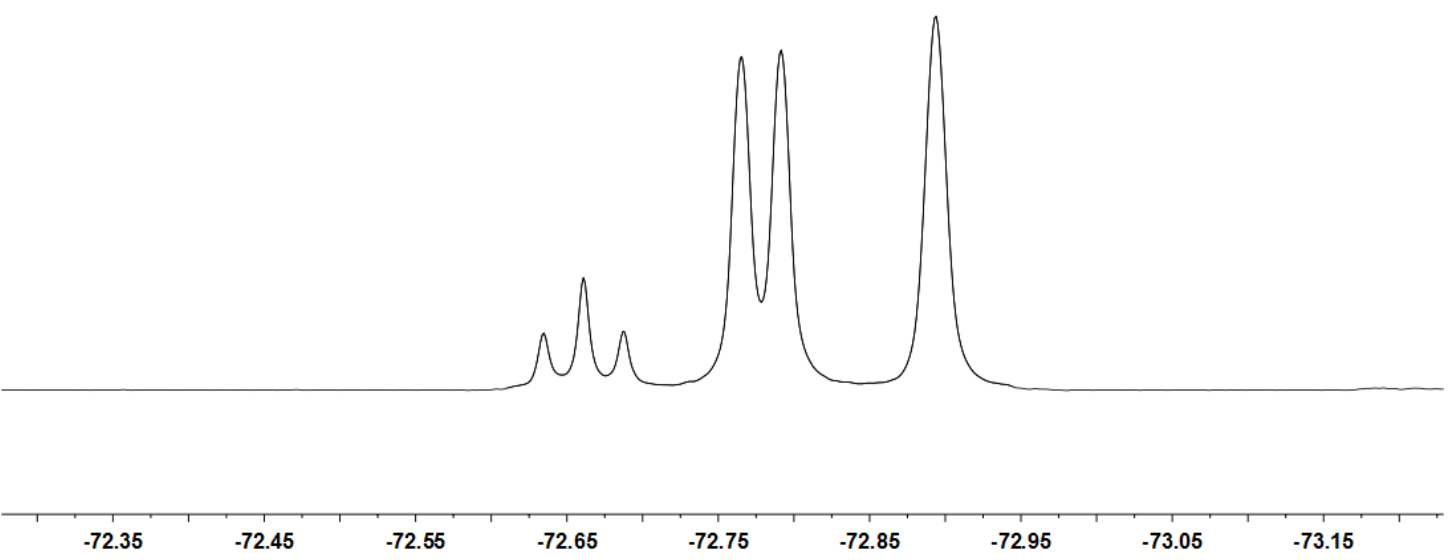

